UNIVERSIDADE DE BRASÍLIA

FACULDADE DE TECNOLOGIA

DEPARTAMENTO DE ENGENHARIA ELÉTRICA

FERRAMENTA COMPUTACIONAL PARA ANÁLISE DE ESTABILIDADE A PEQUENOS SINAIS DE SISTEMAS ELÉTRICOS DE POTÊNCIA

FREDERIQUE AUGUSTO LISBOA RODRIGO DA CUNHA SANTOS

ORIENTADOR: FRANCISCO DAMASCENO FREITAS

MONOGRAFIA DE GRADUAÇÃO EM ENGENHARIA ELÉTRICA

BRASÍLIA/DF: 8 DE SETEMBRO - 2010 

UNIVERSIDADE DE BRASÍLIA

FACULDADE DE TECNOLOGIA

DEPARTAMENTO DE ENGENHARIA ELÉTRICA

\title{
FERRAMENTA COMPUTACIONAL PARA ANÁLISE DE ESTABILIDADE A PEQUENOS SINAIS DE SISTEMAS ELÉTRICOS DE POTÊNCIA
}

\section{FREDERIQUE AUGUSTO LISBOA RODRIGO DA CUNHA SANTOS}

\begin{abstract}
MONOGRAFIA DE GRADUAÇÃO SUBMETIDA AO DEPARTAMENTO DE ENGENHARIA ELÉTRICA DA FACULDADE DE TECNOLOGIA DA UNIVERSIDADE DE BRASÍLIA, COMO PARTE DOS REQUISITOS NECESSÁRIOS PARA A OBTENÇÃO DO GRAU DE ENGENHEIRO ELETRICISTA.
\end{abstract}

APROVADA POR:

Prof. Francisco Damasceno Freitas, Dr. (ENE-UnB)

(Orientador)

Prof. Luis Filomeno de Jesus Fernandes, Dr. (FGA-UnB)

(Examinador Externo)

Eng. Vitor Nunes Nishiyama, (Eletronorte)

(Examinador Externo)

BRASÍLIA/DF, 8 DE SETEMBRO DE 2010 
FICHA CATALOGRÁFICA

LISBOA, FREDERIQUE AUGUSTO e SANTOS, RODRIGO DA CUNHA

Ferramenta Computacional para Análise de Estabilidade

a Pequenos Sinais de Sistemas Elétricos de Potência.

[Distrito Federal] 2010.

xv, 68 páginas, $297 \mathrm{~mm}$ (ENE/FT/UnB, Engenheiro Eletricista, 2010).

Monografia de Gradução - Universidade de Brasília. Faculdade de Tecnologia. Departamento de Engenharia Elétrica.

1. Análise Modal

2. Estabilidade

3. MATLAB

4. Matriz Jacobiana Aumentada

I. $\mathrm{ENE} / \mathrm{FT} / \mathrm{UnB}$

II. Título (série)

\section{REFERÊNCIA BIBLIOGRÁFICA}

LISBOA, F. A. e SANTOS, R. C. (2010). Ferramenta Computacional para Análise de Estabilidade a Pequenos Sinais de Sistemas Elétricos de Potência. Monografia de Gradução, Publicação ENE.MG/2010, Departamento de Engenharia Elétrica, Faculdade de Tecnologia, Universidade de Brasília, Brasília, DF, 71 páginas.

\section{CESSÃO DE DIREITOS}

AUTORES: Frederique Augusto Lisboa

Rodrigo da Cunha Santos.

TÍTULO: Ferramenta Computacional para Análise de Estabilidade a Pequenos Sinais de Sistemas Elétricos de Potência.

GRAU / ANO: Engenheiro Eletricista / 2010

É concedida à Universidade de Brasília permissão para reproduzir cópias desta monografia de graduação e para emprestar ou vender tais cópias somente para propósitos acadêmicos e científicos. O autor reserva outros direitos de publicação e nenhuma parte desta monografia de graduação pode ser reproduzida sem a autorização por escrito do autor.

Frederique Augusto Lisboa

Brasília - DF
Rodrigo da Cunha Santos

Brasília - DF 


\section{DEDICATÓRIA}

Aos meus pais, José Antônio e Heliene, ao meu irmão Thiago e ao meu afilhado Matheus.

Frederique

Aos meus pais, Cristóvão e Maria Divina, e minha irmã, Marina.

Rodrigo 


\section{AGRADECIMENTOS}

Vivemos tempos individualistas, tempos de resignação. A maioria das pessoas pouco se interessa pelo que não lhe diz respeito. Resta, apenas, agradecer àqueles que percebem nossos medos, descrenças, indecisões, suspeitas, e gastam um pouco da sua energia conosco, insistindo e acreditando.

Obrigado mãe por todo esse carinho e dedicação incondicional.

Obrigado pai por sempre acreditar e incentivar a realização deste sonho.

Agradeço aos companheiros de curso e hoje, irmãos, pelos momentos que passamos juntos nesta longa caminhada.

Agradeço, também, à paciência e exemplo profissional do professor e orientador Francisco Damasceno na transmissão de seus ensinamentos.

Frederique 


\section{AGRADECIMENTOS}

Agradeço sobretudo a Deus, que em todos momentos da minha vida me deu forças para alcançar os meus objetivos.

Aos meus pais e minha irmã que tanto os amo, que sempre lutaram para que seus filhos pudessem ter um curso superior. E que sempre me compreendiam, apoiavam e me deram forças nos momentos difíceis que passei.

Aos meus amigos pela consideração e incentivo sempre.

Aos colegas de curso, que passamos nesse período boa parte do tempo juntos, nos tornando uma família.

Agradeço a todas as pessoas que direta ou indiretamente contribuíram de alguma forma para a conclusão deste trabalho.

Rodrigo 


\section{FERRAMENTA COMPUTACIONAL PARA ANÁLISE DE ESTABILIDADE DE SISTEMAS DE PEQUENA POTÊNCIA A PEQUENOS SINAIS.}

\section{AUTORES: RODRIGO DA CUNHA SANTOS FREDERIQUE AUGUSTO LISBOA}

\section{ORIENTADOR: FRANCISCO DAMASCENO FREITAS}

Trabalho de Conclusão de Curso em Engenharia Elétrica - Universidade de Brasília.

BRASÍLIA/DF: 8 DE SETEMBRO - 2010.

Palavras-chave: Estabilidade, Pequenos Sinais, Sistemas de Potência, MATLAB, Sistema de excitação, Sistemas Descritores, Análise Modal, Matriz Jacobiana Aumentada.

\section{RESUMO}

O principal objetivo deste trabalho é apresentar e iniciar o desenvolvimento de um programa para análise linear de estabilidade em sistemas de potência. O programa é desenvolvido totalmente em MATLAB, tendo como base para o cálculo do ponto de operação o programa de fluxo de carga MATPOWER.

Neste Trabalho, o foco é a representação de geradores por meio do seu modelo detalhado e de sistema de excitação e estabilizador de sistemas de potência (ESP) específicos. Teste são efetuados em um sistema de 9 barras e 3 geradores. A estabilidade a pequenos sinais é analisada através do cálculo de autovalores, zeros e resposta no tempo e em frequência. O programa oferece opção de controladores do sistema para ajuste do amortecimento das perturbações. 


\section{Sumário}

1 INTRODUÇÃO 1

2 ESTABILIDADE DE SISTEMAS DE POTÊNCIA 4

2.1 Estabilidade de Sistemas Dinâmicos . . . . . . . . . . . . . . . . . 4

2.2 Limites de Estabilidade em Sistemas de Potência [4] . . . . . . . . . . . 6

2.3 Definição de Estabilidade do Sistema de Potência . . . . . . . . . . . . 6

2.4 Sistemas Lineares e Não-Lineares $[1,4] \ldots \ldots$. . . . . . . . . . . . 8

2.5 Linearização de Sistemas Não-lineares . . . . . . . . . . . . . . . . . . . 9

3 MODELAGEM E MÉTODOS DE ANÁLISE DO SEP 12

3.1 Contextualização . . . . . . . . . . . . . . . . . . . . . . . 12

3.2 Métodos de Construção do Modelo Dinâmico . . . . . . . . . . . . . . . 16

3.2 .1 Espaço de Estados . . . . . . . . . . . . . . . . . 16

3.2 .2 Sistemas Descritores _. . . . . . . . . . . . . . . 18

3.3 Representação Linear do SEP . . . . . . . . . . . . . . . . . . . . . . . 19

3.3.1 Condições Iniciais . . . . . . . . . . . . . . . . . . . . . . . . 20

3.3.2 Equações dos Geradores . . . . . . . . . . . . . . . . . . . . 21

3.3.3 Sistema de Excitação . . . . . . . . . . . . . . . . . . . . . . 24

3.4 Métodos de Análise do Modelo Dinâmico . . . . . . . . . . . . . . . 27

3.4.1 Autovalores, Autovetores e Transformações . . . . . . . . . . . . 27

3.4.2 Critério de Estabilidade de Nyquist . . . . . . . . . . . . . . 30

4 MODELAGEM DE CARGAS 32

4.1 Contextualização . . . . . . . . . . . . . . . . . . . . . . . . . . . 32

4.2 Conceitos Básicos de Modelagem de Carga . . . . . . . . . . . . . . . . 32

4.3 Modelagem de Cargas Estáticas Não-Lineares . . . . . . . . . . . . . 35

5 REPRESENTAÇÕES, ANÁLISES E RESULTADOS 40

5.1 Introdução . . . . . . . . . . . . . . . . . . . . . . . . 40

5.2 Formação da Matriz Jacobiana . . . . . . . . . . . . . . . . . . . . . 43

5.3 Obtenção do Sistema Descritor . . . . . . . . . . . . . . . . . . . . . 44 
5.4 Análise Modal, da Resposta no Tempo e em Frequência . . . . . . . . . 45

6 CONCLUSÕES E SUGESTÕES PARA TRABALHOS FUTUROS 49 $\begin{array}{ll}\text { APENDICE } & 52\end{array}$

A ESQUEMÁtico MATRIZ A PARA O CASE 9

B CÓDIGO MATLAB IMPLEMENTADO 58 


\section{Lista de Tabelas}

3.1 Símbolos utilizados no modelo \#6 . . . . . . . . . . . . . . . . . . . . . 14

5.1 Dados de ligacão . . . . . . . . . . . . . . . . . . . . . . 40

5.2 Dados de barra . . . . . . . . . . . . . . . . . . . . . . . 41

5.3 Resultados do Fluxo de carga . . . . . . . . . . . . . . . . . 42

5.4 Dados dos geradores e controladores do sistema em estudo - Parte 1 . . 42

5.5 Dados dos geradores e controladores do sistema em estudo - Parte 2 . . 42

5.6 Resultados obtidos para o case9 . . . . . . . . . . . . . 45 


\section{Lista de Figuras}

2.1 Linearização sobre um ponto de operação [4] . . . . . . . . . . . . . . . 9

3.1 Fluxograma para obtenção da representação linear do SEP [6] . . . . 20

3.2 Sistema de excitação de um gerador síncrono [1] . . . . . . . . . . . . . 25

3.3 Sistema de excitação típico, incluindo sinal adicional produzido pelo PSS 25

3.4 Bloco lead - lag utilizado como função de transferência do PSS (não está sendo considerado o bloco washout nesta modelagem) . . . . . . . 26

3.5 Modelo do PSS com o bloco lead - lag desmembrado em um ganho e um bloco de atraso de primeira ordem . . . . . . . . . . . . . . . 26

3.6 Representação em diagrama de blocos de um sistema de controle realimentado . . . . . . . . . . . . . . . . . . . . 30

4.1 Configuração do Sistema Elétrico de Potência enfatizando as cargas presentes no sistema a partir da barra de carga A [1] . . . . . . . . . . 33

4.2 Injeção de corrente na barra de carga . . . . . . . . . . . . . . . . 35

5.1 Sistema de nove barras da referência [2] . . . . . . . . . . . . . . . . 41

5.2 Arranjo físico da matriz Jacobiana para o case 9 . . . . . . . . . . . . 43

5.3 Interface gráfica de entrada de dados . . . . . . . . . . . . . . . . 45

5.4 Resposta a um degrau para o case9 . . . . . . . . . . . . . . . 47

5.5 Zoom aplicado em regime permanente . . . . . . . . . . . . . . 47

5.6 Diagrama de Nyquist plotado com a saída do projeto . . . . . . . . . . 48

5.7 Diagrama de Nyquist plotado com a saída do PACDyN . . . . . . . . . 48 


\section{LISTA DE SÍMBOLOS}

\section{Símbolos Latinos}

$A, B, C, D \quad$ Matrizes de estado, entrada, saída e de transmissão direta

$D_{t} \quad$ Constante de amortecimento do gerador síncrono

$E_{d}^{\prime} E_{q}^{\prime} E_{d}^{\prime \prime} E_{q}^{\prime \prime}$ Tensões internas de eixo direto $(d)$ e quadratura $(q)$, transitória e subtransitória da máquina síncrona

$V_{d}, V_{q} \quad$ Tensão do gerador nos eixos $d$ e $q$

$I_{d}, I_{q} \quad$ Corrente de armadura nos eixos $d$ e $q$

$I_{i} \quad$ Fluxo de corrente na barra terminal da máquina $i$

$X_{l} \quad$ Reatância de dispersão

$P_{t} \quad$ Potência ativa terminal na máquina síncrona

$P_{e} \quad$ Potência elétrica

$T_{e} \quad$ Torque elétrico

$T_{m} \quad$ Torque mecânico no eixo da turbina

$H \quad$ Constante de inércia em $\frac{M W \cdot s}{M V A}$

$R_{a} \quad$ Resistência da armadura

$P_{c}, Q_{c} \quad$ Potência ativa e reativa da carga

$P_{e}, P_{m} \quad$ Potências elêtrica de saída e mecânica de entrada do gerador

$S_{d}, S_{q} \quad$ Potência aparente de eixo $d$ e $q$

$S_{i} \quad$ Potência nominal do i-ésimo gerador

$X_{d}, X_{q} \quad$ Reatância síncrona de eixo $d$ e $q$

$X_{d}^{\prime}, X_{q}^{\prime} \quad$ Reatância transitória de eixo $d$ e $q$

$X_{d}^{\prime \prime}, X_{q}^{\prime \prime} \quad$ Reatância subtransitória de eixo direto e em quadratura

$T_{d 0}^{\prime}, T_{q 0}^{\prime} \quad$ Constante de tempo tansitória da máquina síncrona de circuito aberto dos eixos $d$ e $q$

$T_{d 0}^{\prime \prime} \quad$ Constante de tempo subtansitória da máquina síncrona de circuito aberto do eixos $d$

$f \quad$ Frequência em Hertz

$V_{t} \quad$ Tensão terminal do gerador

$E_{f d} \quad$ Tensão de campo do gerador

$a, b, c, d \quad$ Parâmetros do modelo polinomial de representação de cargas 
$K_{c i 1}, K_{c i 2} \quad$ Constantes referentes a modelagem de cargas para potência ativa

$K_{c i 3}, K_{c i 4} \quad$ Constantes referentes a modelagem de cargas para potência reativa

$P_{R E F} \quad$ Potência aparente referência no gerador

$V_{r i}, V_{m i} \quad$ Tensão nos eixos $d$ e $q$

$V_{R E F} \quad$ Tensão referência no gerador

$K_{a}, T_{a} \quad$ Constantes de tempo para dispositivos Regulador de Tensão (RT)

$V_{R E F}^{R T}, V_{t}^{R T} \quad$ Variáveis de estado do RT

$V_{P S S}^{R T}, X_{0005}^{R T} \quad$ Variáveis de estado do RT

$E_{f d}^{R T}, X_{1}^{R T} \quad$ Variáveis de estado do RT

$V_{P P S} \quad$ Tensão de saída do Estabilizador de Sistema de Potência (ESP)

$V_{P P S}^{P P S}, X_{0006}^{P S S} \quad$ Variáveis de estado do ESP

$X_{1}^{P S S} \quad$ Variável de estado do ESP

$D \omega^{P S S} \quad$ Variável de estado do ESP

$T_{1}, T_{2} \quad$ Constantes de tempo para dispositivos ESP

$K_{P S S} \quad$ Ganho do Estabilizador de Sistema de Potência

\section{Símbolos Gregos}

$\delta$

$\Delta$

$\lambda_{i}$

$\theta$

$\omega$

$\omega_{o}$

$\omega \omega$

$\partial$
Ângulo das barras internas do gerador

Variação entre duas grandezas similares

Autovalores, que são iguais à variancia do comportamento de cada gerador

Ângulo das barras terminais do gerador obtidos de uma solução de fluxo de carga

Velocidade ângular do rotor do gerador

Velocidade síncrona do sistema igual a $2 \pi f$ em $\frac{\mathrm{rad}}{\mathrm{s}}$

Velocidade angular

Derivada Parcial

Operador Derivada 


\section{Siglas}

CEPEL Centro de Pesquisas de Energia Elétrica

SEP Sistema Elétrico de Potência

ESP Estabilizador de Sistema de Potência

FACTS Sistema de Transmissão Flexível de Corrente Alternada (Flexible AC Transmission System).

RT Regulador de Tensão

$\mathrm{pu} \quad$ Por unidade

AnAREDE Programa de Análise de Redes

AnAtem Programa de Análise de Transitórios Eletromecânicos

PACDyn Programa de Análise Linear e Controle do Amortecimento de Oscilações em Sistemas de Potência

MATLAB Software interativo de alta performance voltado para o cálculo numérico

Matpower Software desenvolvido em Matlab para o cálculo do fluxo de potência

PSS Estabilizadores de Sistemas de Potência (Power System Stabilizers)

SVC Compensadores Estáticos de Reativos (Static Var Compensators)

EPRI Instituto de Pesquisa de Potência Elétrica (Eletric Power Research Institute)

ZIP Modelo polinomial de representação de cargas estáticas não-lineares 


\section{Capítulo 1 INTRODUÇÃO}

A crescente demanda por energia elétrica, junto com a complexidade da operação e controle do sistema de energia elétrica requer a otimização de sistemas de monitoramento e controle para garantir a operação da rede elétrica em conformidade com os critérios de continuidade, confiabilidade e qualidade de suprimento.

A análise de estabilidade de Sistemas Elétricos de Potência (SEP) é essencial para o contínuo fornecimento de energia elétrica. Entretanto, todo sistema está sujeito a condições adversas e imprevisíveis que podem levar a situações de falha ou inadequada operação tornando-o instável. A instabilidade pode ser gerada por perturbações, sejam grandes perturbações (quando se refere à estabilidade transitória) ou pequenas perturbações (quando se refere à estabilidade dinâmica).

Apartir do final da década de 50, novos geradores equipados com reguladores de tensão de ação contínua foram introduzidos nos sistemas elétricos de potência. Devido aos benefícios provenientes da utilização desses dispositivos, em pouco tempo, a maioria dos geradores já dispunha dessa facilidade. Esse fato, aliado à crescente interligação dos sistemas, deu origem ao fenômeno de oscilações eletromecânicas de baixa frequência decorrente de interações dinâmicas entre os geradores, e mais evidente como oscilações de fluxos de potência sincronizante na rede de transmissão. As oscilações do ângulo do rotor do gerador síncrono, também conhecidas como modos de oscilações eletromecânicas do sistema elétrico, podem ser excitados por estas pequenas perturbações. As oscilações eletromagnéticas surgem no sistema elétrico em regime permanente, em condição de carga pesada, aliadas a longas linhas de transmissão e possuem frequências na faixa de 0,1 a $2,0 \mathrm{~Hz}[5,3]$.

$\mathrm{Na}$ faixa de frequência em questão, o amortecimento natural do sistema é bastante reduzido o que favorece o aparecimento de oscilações fracamente amortecidas ou até com amplitudes crescentes ameaçando ou inviabilizando a operação estável de sistemas 
interligados.

A resposta transitória dos sistemas elétricos de potência, em geral, é caracterizada por oscilações eletromecânicas de baixa frequência, que surgem a partir da falta de torque de amortecimento em sistemas elétricos. Estas oscilações são prejudiciais para a operação dos sistemas elétricos de potência, pois as mesmas podem dificultar a operação do sistema em condições transitórias. Tais oscilações podem, em alguns casos, gerar limitações a capacidade de transmissão de potência elétrica entre as barras do sistema, devido às variações nos fluxos de potência nas linhas de transmissão. Além disto, as oscilações de baixa frequência, quando mal amortecidas, podem causar a perda de sincronismo dos geradores. Reguladores de tensão com constantes de tempo pequenas e altos ganhos aliados às condições de grandes potências transferidas a longas distâncias, comprometem ainda mais a questão da estabilidade às pequenas pertubações [5].

No entanto, os efeitos adversos causados pelas oscilações podem ser reduzidos através do amortecimento das mesmas, introduzindo sinais adicionais estabilizantes nos sistemas de excitação para fornecer torque de amortecimento. Além de estender o limite de transferência de potência, em condições transitórias, o amortecimento das oscilações proporciona uma operação estável e segura dos sistemas elétricos de potência submetidos a pequenas perturbações, permitindo assim o contínuo fornecimento de energia.

Para se analisar o problema de estabilidade relativo ao amortecimento de oscilações eletromecânicas utiliza-se um modelo linearizado do sistema a partir de um ponto de equilíbrio e o modelo matemático resultante é formado por um conjunto de equações diferencias lineares, invariantes no tempo. O método utilizado na modelagem e representações reflete no grau de detalhamento dos modelos linearizados. Um dos modelos classicamente utilizados quando se trata de estabilidades a pequenos sinais é o modelo de Heffron - Phillips [5, 9]. Neste modelo, a máquina síncrona é representada por três equações diferenciais, além de uma equação diferencial adicional relativa ao seu sistema de excitação [8]. Esse modelo apresenta algumas limitações quanto à representação da máquina, dificultando modelagens mais detalhadas, a representação de cargas, inclusão de equipamentos FACTS. Com o objetivo de superar as limitações intrínsecas ao modelo Heffron - Phillips e consequentemente facilitar a inclusão 
de outros dispositivos na modelagem do sistema elétrico, foi proposto um modelo alternativo, para o sistema de potência, denominado modelo da matriz Jacobiana aumentada, composto de variáveis de estado e algébricas, que não apresenta as limitações citadas antes. Com isso, a estrutura altamente esparsa da matriz Jacobiana permite o emprego de técnicas para solução de sistemas lineares, e a exploração de técnicas de esparsidade. Além disso, há maior flexibilidade quanto à representação de cargas, elos de corrente contínua, equipamentos FACTS, etc.

Investimentos em pesquisa e desenvolvimento de novas técnicas, estudos e tecnologias crescem visando melhorar o fornecimento de energia. Constantemente são desenvolvidas novas ferramentas para que se possa melhorar os padrões, confiabilidade e continuidade no fornecimento.

Este trabalho tem como objetivo investigar a estabilidade às pequenas perturbações de um sistema elétrico de potência e por meio de um programa desenvolvido no MATLAB. Neste sentido, procura-se responder/reproduzir problemas do tipo [6]:

$\diamond$ Identificação do ponto de operação estável do SEP.

$\diamond$ Operação segura do SEP em determinada condição degradada.

$\diamond$ Controle do amortecimento de oscilações.

Este trabalho está organizado da seguinte forma: no Capítulo 2 são apresentados conceitos relativos a modelos de sistemas e estabilidade. No Capítulo 3 são apresentados os métodos de construção e análise do modelo dinâmico assim como a representação linear do SEP. No Capítulo 4, conceitos básicos de modelagem de cargas e o estudo de cargas estáticas não-lineares são abordados. No Capítulo 5 foram realizadas simulações e análises de resultados de um caso prático obtidas a partir da formulação do problema apresentado nos capítulos anteriores. No Capítulo 6 são apresentadas as conclusões e sugestões para trabalhos futuros. No Apêndice A é apresentado detalhadamente a formação da matriz Jacobiana aumentada para o caso abordado no projeto. No Apêndice B, pode-se encontrar o código fonte do programa principal implementado no projeto. 


\section{Capítulo 2 ESTABILIDADE DE SISTEMAS DE POTÊNCIA}

Um modelo matemático que descreve as operações de um sistema de energia contém equações diferenciais e algébricas. Um sistema de potência, como qualquer outro sistema dinâmico, é normalmente sujeito a perturbações contínuas. Por conveniência, podemos assumir que a nível operacional, o sistema está em repouso, ou seja, está em um ponto de equilíbrio. Isto implica que podemos encontrar uma solução de estado estacionário para as equações que descrevem o funcionamento do sistema de potência. Além disso, a fim de ser viável, os estados do sistema em equilíbrio devem ficar dentro de limites aceitáveis $[1,4]$.

\subsection{Estabilidade de Sistemas Dinâmicos}

Considere um sistema dinâmico representado pela equação diferencial do vetor $\mathbf{x}$ da forma geral:

$$
\dot{\mathbf{x}}=\mathbf{f}(\mathbf{x}, \mathbf{u}, t)
$$

onde,

$$
\mathbf{x}=\left[\begin{array}{c}
x_{1} \\
x_{2} \\
\vdots \\
x_{n}
\end{array}\right] \mathbf{u}=\left[\begin{array}{c}
u_{1} \\
u_{2} \\
\vdots \\
u_{r}
\end{array}\right] \mathbf{f}=\left[\begin{array}{c}
f_{1} \\
f_{2} \\
\vdots \\
f_{n}
\end{array}\right]
$$

em que $\mathbf{x}$ é referido como o vetor de estado do sistema, e suas entradas $x_{n}$ como variáveis de estado. O vetor coluna u é o vetor de entrada para o sistema. Já $n$ é a ordem do sistema e $r$ é o número de entradas. Se as derivadas das variáveis de estado não são funções explícitas do tempo, $t$, então o sistema é dito ser autônomo e a equação (2.1) assume uma forma mais simples [1, 4]: 


$$
\dot{\mathbf{x}}=\mathbf{f}(\mathbf{x}, \mathbf{u})
$$

Em geral, o conjunto de equações diferênciais não-lineares que descrevem a dinâmica de um sistema de potência reduz-se a esta forma.

\section{Pontos de Equilíbrio}

Os pontos de equilíbrio são os pontos em que todas as derivadas $\dot{x_{1}}, \dot{x_{2}}, \ldots, \dot{x_{n}}$ são simultâneamente zero; eles definem os pontos da trajetória com velocidade zero. $\mathrm{O}$ sistema, está em repouso, desde que todas as variáveis sejam constantes e invariáveis com o tempo.

O ponto de equilíbrio deve satisfazer a equação:

$$
\mathbf{f}\left(\mathbf{x}_{\mathbf{0}}\right)=0
$$

onde $\mathbf{x}_{\mathbf{0}}$ é o vetor de estado $\mathbf{x}$ no ponto de equilíbrio.

Se as funções $f_{i}(i=1,2, \ldots, n)$ na equação (2.3) são lineares, então o sistema é linear. Um sistema linear tem apenas um estado de equilíbrio. Para um sistema não-linear, pode haver mais de um ponto de equilíbrio.

Os pontos singulares possuem as características do comportamento do sistema dinâmico e, portanto, é possível tirar conclusões sobre a estabilidade de sua natureza [1]. 


\subsection{Limites de Estabilidade em Sistemas de Potência [4]}

\section{Limite de Estabilidade de Regime Permanente}

Trata-se de uma condição de operação para a qual o sistema de potência é estável em regime permanente, mas que uma pequena variação arbritrária em qualquer das variáveis de operação, em uma direção desfavorável provoca a perda de estabilidade do sistema. Isto também é conhecido como Limite de Estabilidade a Pequena Perturbação.

\section{Limite de Estabilidade Transitória}

O limite de estabilidade transitória para uma perturbação particular é a condição de operação em regime permanente para o qual o sistema de potência é transitoriamente estável, mas para a qual uma variação arbritrária em qualquer das variáveis de operação, em uma direção desfavorável, leva o sistema a perder a estabilidade para aquela perturbação.

\subsection{Definição de Estabilidade do Sistema de Potência}

Um sistema de potência em um determinado estado de funcionamento é estável se após uma perturbação, ou um conjunto de pertubações, o estado do sistema permanece dentro dos limites especificados e o sistema atinge um novo estado de equilíbrio estável dentro de um período de tempo especificado.

Estabilidade, tal como definido acima, inclui todos os tipos de estabilidade que poderão ser encontrados em sistemas de potência. Exemplos são: a estabilidade síncrona (eletromecânica ou angular), estabilidade de torção do eixo, estabilidade de tensão, estabilidade de frequência, etc. Para exemplo, se a estabilidade em questão envolve apenas o sincronismo angular, a definição acima deve ser atualizado como se segue: 
Definição 2.3.1 Um sistema de potência em um determinado estado de funcionamento e sujeito a perturbação apresenta estabilidade eletromecânica quando existe a manutenção de sincronismo dos geradores do sistema. Este fenômeno é fortemente associado ao balanço de potência ativa (MW). Engloba análise de estabilidade oscilatória (ou dinâmica) e transitória (ou de primeira oscilação) [3].

A outra razão para não definir os diferentes tipos de estabilidade em separado é que se o sistema é considerado estável, deve ser estável em todos os sentidos. Ou seja, um sistema de potência que possui estabilidade síncrona, mas não estabilidade de tensão, não é aceitável. No entanto, por motivos práticos, é conveniente conhecer o modo de instabilidade quando o sistema se torna instável para que medidas corretivas possam ser planejadas e implementadas. Duas principais classes de estabilidade na dinâmica do sistema de potência são: estabilidade a pequenas e grandes perturbações.

\section{Estabilidade a Pequenas Perturbações}

Se a magnitude da perturbação é suficientemente pequena para que a resposta do sistema na fase inicial seja essencialmente linear, a estabilidade pode ser classificada como estabilidade a pequenas perturbações. Como será visto posteriormente a estabilidade de pequenas perturbações é assegurada se os autovalores do modelo dinâmico linear sobre o ponto de equilíbrio, têm partes reais negativas.

Apesar das partes reais negativas dos autovalores do sistema linearizado proporcionarem condições suficientes para a estabilidade de pequenas perturbações, em algumas situações, outros critérios mais simples podem ser aplicáveis. Informações sobre a estabilidade de pequenas perturbações podem também ser obtidos a partir de uma solução das equações não-lineares usando uma pequena perturbação, mas finita. 


\section{Estabilidade a Grandes Perturbações}

A estabilidade de grandes perturbações é caracterizada pela perda do sincronismo de qualquer uma das máquinas do sistema elétrico, quando a magnitude das perturbações pode levar o sistema a regiões de não-linearidade. Também é chamado de estabilidade transitória.

Este trabalho irá focar o estudo da estabilidade dinâmica de pequenas perturbações.

\subsection{Sistemas Lineares e Não-Lineares $[1,4]$}

Um sistema é definido como linear, em termos do sistema de excitação e de sua resposta. Em geral, uma condição necessária para um sistema linear pode ser determinada em termos de uma excitação $x(t)$ e uma resposta $y(t)$. Suponha que o sistema em repouso é submetido a uma excitação $x_{1}(t)$ e o resultado é uma resposta $y_{1}(t)$. Também suponha que, quando submetido a uma excitação $x_{2}(t)$ o resultado é uma resposta correspondente $y_{2}(t)$. Para um sistema linear, é necessário que a excitação $x_{1}(t)+x_{2}(t)$ resulte em uma resposta $y_{1}(t)+y_{2}(t)$. Isto é chamado de princípio da superposição. Além disso, é necessário que o fator de escala da magnitude seja preservada em um sistema linear. Novamente, consideramos um sistema com uma entrada $x$ que resulta em uma saída $y$. Então, se uma entrada $x$ for multiplicada por uma constante $\beta$, tendo assim $\beta x$, é necessário que a saída seja multiplicada pela mesma constante, ou seja, $\beta y$. Esta é chamada de propriedade da homogeneidade. Um sistema é linear se, e somente se, as propriedades de superposição e homogeneidade são satisfeitas.

Um sistema caracterizado pela relação $y=x^{2}$ não é linear, uma vez que a superposição de propriedade não é satisfeita. Um sistema que é representado pela relação $y=m x+$ $b$ não é linear, uma vez que não satisfaz a propriedade da homogeneidade. No entanto, ambos os sistemas podem ser considerados lineares sobre um ponto de operação $x_{o}, y_{o}$ para pequenas variações $\Delta x$ e $\Delta y$. 


\subsection{Linearização de Sistemas Não-lineares}

Caso um sistema não seja linear, ele pode ser linearizado sobre um ponto de operação, assumindo-s pequenas variações. Considere um elemento geral não-linear com uma variável de excitação $x(t)$ e a variável resposta $y(t)$, a relação entre as duas variáveis é dada como:

$$
y(t)=f(x(t))
$$

A relação pode ser apresentado graficamente como na Figura 2.1. O ponto de operação normal é designada por $x_{o}, y_{o}$. Como a curva da função é contínua em todo o alcance de interesse, uma expansão da série de Taylor sobre o ponto de operação pode ser utilizada. Então temos:

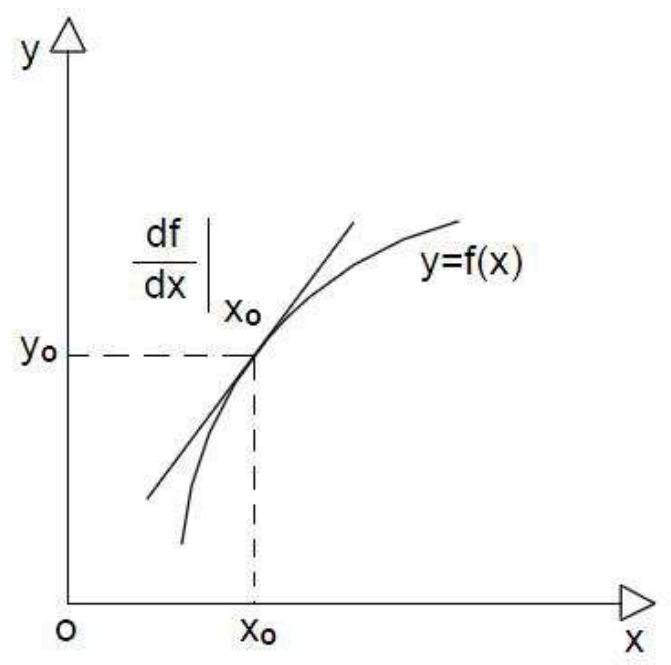

Figura 2.1: Linearização sobre um ponto de operação [4]

$$
y=f(x)=f\left(x_{o}\right)+\frac{f^{\prime}\left(x_{o}\right)}{1 !} \Delta x+\frac{f^{\prime \prime}\left(x_{o}\right)}{2 !}(\Delta x)^{2}+\ldots
$$

onde,

$$
\Delta x=x-x_{o}
$$


Para $\Delta x$ pequeno, os termos de ordem superior podem ser desprezadas. Portanto,

$$
y=f\left(x_{o}\right)+f^{\prime}\left(x_{o}\right) \Delta x
$$

que produz,

$$
\Delta y=f^{\prime}\left(x_{o}\right) \Delta x
$$

onde,

$$
\Delta y=y-f\left(x_{o}\right)=y-y_{o}
$$

e,

$$
f^{\prime}\left(x_{o}\right)=\left.\frac{d f}{d x}\right|_{x_{0}}
$$

é a inclinação no ponto de operação.

A mesma técnica pode ser estendida para sistemas de diversas variáveis, representada por uma equação do vetor da forma:

$$
\mathbf{y}=f(\mathbf{x})
$$

Onde y é um vetor de $n$ variáveis dependentes $y_{1}, y_{2}, \ldots, y_{n}$ e $f(\mathbf{x})$ é uma função de $n$-vetores das variáveis de excitação $x_{1}, x_{2}, \ldots, x_{m}$.

Empregando expansão da série de Taylor sobre o ponto de operação $x_{1 o}, x_{2 o}, \ldots, x_{m o}$, e desprezando os termos de ordem superior, obtemos o sistema linear como: 


$$
\left[\begin{array}{c}
\Delta y_{1} \\
\Delta y_{2} \\
\vdots \\
\Delta y_{n}
\end{array}\right]=\left[\begin{array}{cccc}
\frac{\partial f_{1}(x)}{\partial x_{1}} & \frac{\partial f_{1}(x)}{\partial x_{2}} & \ldots & \frac{\partial f_{1}(x)}{\partial x_{m}} \\
\frac{\partial f_{2}(x)}{\partial x_{1}} & \frac{\partial f_{2}(x)}{\partial x_{2}} & \ldots & \frac{\partial f_{2}(x)}{\partial x_{m}} \\
\vdots & \vdots & \vdots & \vdots \\
\frac{\partial f_{n}(x)}{\partial x_{1}} & \frac{\partial f_{n}(x)}{\partial x_{2}} & \ldots & \frac{\partial f_{n}(x)}{\partial x_{m}}
\end{array}\right]+\left[\begin{array}{c}
\Delta x_{1} \\
\Delta x_{2} \\
\vdots \\
\Delta x_{m}
\end{array}\right]
$$

que pode ser escrita como:

$$
\Delta y=\mathbf{J} \Delta x
$$

Em que $\mathbf{J}$ é chamado de jacobiano do sistema. 


\section{Capítulo 3 MODELAGEM E MÉTODOS DE ANÁLISE DO SEP}

\subsection{Contextualização}

Neste capítulo iremos realizar uma análise do comportamento dinâmico do sistema elétrico de potência e neste sentido focaremos a estabilidade dinâmica, ou seja, a estabilidade a pequenas perturbações. Em muitos casos, instabilidades e perdas de sincronismo são iniciadas por algumas perturbações espúrias que se não forem amortecidas podem provocar o colapso do sistema. Oscilações, mesmo que pequenas, são indesejáveis ao passo que elas podem limitar a transmissão de potência em linhas de transmissão e, às vezes, induzem estresse desnecessário ao eixo mecânico da máquina síncrona.

Em grandes sistemas de energia elétrica ocorrem diversos tipos de oscilações como, por exemplo, modos de oscilação devidos aos sistemas de controle da excitação e de velocidade da máquina síncrona. Os principais modos para o estudo da estabilidade a pequenas perturbações são aqueles associados às oscilações dos rotores das máquinas síncronas, que são denominados de "modos eletromecânicos de oscilação". Tais oscilações são preponderantes em grandes sistemas interligados, em que o transporte de energia é realizado por longas linhas de transmissão com altas reatâncias indutivas. Para o estudo de seus efeitos no comportamento dinâmico do sistema elétrico necessitase de uma modelagem detalhada. Elas podem ser classificadas de acordo com sua freqüência de oscilação, sendo os de maior interesse os modos locais e os modos interárea.

Modos locais de oscilação Encontram-se na faixa de 0,7 a 2,0 Hz e estão associados às oscilações dos rotores de um grupo de geradores próximos, fisicamente ou eletricamente [1].

Modos interárea de oscilação Localizam-se na faixa de 0,1 a $0,8 \mathrm{~Hz}$ e são relacionados com as oscilações de grupos de geradores de uma área contra outro grupo de 
geradores de outra área [1].

Atualmente inúmeras pesquisas estão sendo direcionadas a estas duas áreas. Oscilações de modo local tendem a ocorrer quando geradores (ou grupo de geradores) são conectados ao resto do sistema através de linhas de transmissão que possuem altos valores de impedância, como é o caso das grandes usinas hidrelétricas situadas distantes dos seus centros de consumo e, portanto conectadas por longas linhas de transmissão (essencialmente radiais) em alta e extra-alta tensão. Oscilações de modo interárea ocorrem principalmente quando os sistemas são conectados por linhas relativamente fracas, ou seja, com capacidades muito inferiores às capacidades dos sistemas que elas interligam. Transferências regionais de potência variam ao longo do tempo devido a fatores como a reestruturação e o livre acesso ao sistema de transmissão o que faz com que certas partes do sistema enfrentem condições de carga aumentada. Oscilações de modo interárea são mais difíceis de serem estudas e amortecidas, pois elas são influenciadas por estados globais, e uma análise detalhada de todo o sistema interligado é necessária para estudar esse fenômeno.

Rotineiramente este fenômeno era analisado segundo considerações estáticas, ou seja, equações do fluxo de carga. Neste capítulo iremos desenvolver um modelo dinâmico que será utilizado no estudo de oscilações a baixas frequências e da estabilidade dinâmica de tensão. Para tanto utilizaremos um modelo $(\# 6)$ de máquina síncrona que representa os efeitos transitórios (campo e enrolamento) e subtransitórios no eixos direto $(d)$ e em quadratura $(q)$ mas não considera a influência da mudança na frequência do sistema na variação dos parâmetros e tensões da máquina. O modelo \#6 é apresentado pelas seguintes equações [6]:

\section{Equações Diferenciais}

$$
\begin{aligned}
& \frac{d E_{d}^{\prime \prime}}{d t}=\frac{1}{T_{q 0}^{\prime \prime}}\left[-E_{d}^{\prime \prime}+\left(X_{q}-X_{q}^{\prime \prime}\right) I_{q}\right] \\
& \frac{d E_{q}^{\prime}}{d t}=\frac{1}{T_{d 0}^{\prime}}\left[E_{f d}+\frac{X_{d}-X_{d}^{\prime}}{X_{d}^{\prime}-X l} E_{q}^{\prime \prime}-\frac{X_{d}-X_{l}}{X_{d}^{\prime}-X_{l}} E_{q}^{\prime}-\frac{\left(X_{d}-X_{d}^{\prime}\right)\left(X_{d}^{\prime \prime}-X_{l}\right)}{X_{d}^{\prime}-X_{l}} I_{d}-S A T\right] \\
& \frac{d E_{q}^{\prime \prime}}{d t}=\frac{1}{T_{d 0}^{\prime \prime}}\left[-E_{q}^{\prime \prime}+E_{q}^{\prime}-\left(X_{d}^{\prime}-X_{d}^{\prime \prime}\right) I_{d}\right]+\frac{X_{d}^{\prime \prime}-X_{l}}{X_{d}^{\prime}-X_{l}} \frac{d E_{q}^{\prime}}{d t}
\end{aligned}
$$




$$
\begin{aligned}
& 2 H \frac{d \omega_{r}}{d t}+D_{t} \omega_{r}=T_{m}-T_{e} \\
& \frac{d \delta}{d t}=\omega_{r}-\omega_{s}
\end{aligned}
$$

Equações Algébricas

$$
\begin{aligned}
& S A T=A e^{B\left|E_{q}^{\prime}\right|-C} \\
& V_{d}=E_{d}^{\prime}+X_{q}^{\prime} I_{q}-R_{a} I_{d} \\
& V_{q}=E_{q}^{\prime}-X_{d}^{\prime} I_{d}-R_{a} I_{q} \\
& V_{t}^{2}=V_{d}^{2}+V_{q}^{2} \\
& P_{t}=V_{d} I_{q}+V_{q} I_{q} \\
& P_{e}=P_{t}+R_{a}\left(I_{d}^{2}+I_{q}^{2}\right)
\end{aligned}
$$

Tabela 3.1: Símbolos utilizados no modelo \#6

\section{Símbolo Descrição}

\section{Unidade}

$\begin{array}{clc}V_{d} V_{q} & \begin{array}{l}\text { Tensões de eixo direto e quadratura no terminal } \\ \text { da máquina síncrona. }\end{array} & \mathrm{pu} \\ V_{t} & \text { Tensão terminal da máquina síncrona. } & \mathrm{pu} \\ I_{d} I_{q} & \text { Correntes de eixo direto e quadratura no terminal } & \mathrm{pu} \\ & \text { da máquina síncrona. } & \\ E_{d}^{\prime} E_{q}^{\prime} E_{d}^{\prime \prime} E_{q}^{\prime \prime} & \text { Tensões internas de eixo direto e quadratura, } & \mathrm{pu} \\ & \text { transitório e subtransitório da máquina síncrona. } & \\ T_{d 0}^{\prime} T_{q 0}^{\prime} T_{d 0}^{\prime \prime} & \text { Constante de tempo de circuito aberto de eixo } & \mathrm{s} \\ & \text { direto e quadratura, transitório e subtransitório da } & \\ X_{d}^{\prime} X_{d}^{\prime \prime} X_{q}^{\prime \prime} & \text { máquina síncrona. } & \\ & \text { Reatância de eixo direto e quadratura, transitório } & \mathrm{pu} \\ X_{l} & \text { Reatância de dispersão } & \\ E_{f d} & \text { Tensão de campo } & \mathrm{pu} \\ P_{t} & \text { Potência ativa terminal na máquina síncrona. } & \mathrm{pu} \\ P_{e} & \text { Potência elétrica. } & \mathrm{pu} \\ T_{e} & \text { Torque elétrico. } & \mathrm{pu} \\ & & \mathrm{pu}\end{array}$


Tabela 3.1 - Símbolos utilizados - Continuação

\begin{tabular}{clc}
\hline Símbolo & Descrição & Unidade \\
\hline$T_{m}$ & Torque mecânico no eixo da turbina. & $\mathrm{pu}$ \\
$\delta$ & Posição angular do rotor da máquina síncrona em & $\mathrm{rad}$ \\
& relação à uma referência que gira na velocidade & \\
& síncrona. & $\mathrm{rad} / \mathrm{s}$ \\
$\omega_{s}$ & Velocidade síncrona. & $\mathrm{rad} / \mathrm{s}$ \\
$\omega_{r}$ & Velocidade angular do rotor da máquina referenci- & \\
& ada à velocidade síncrona & $\mathrm{W} . s / M V A$ \\
$H^{*}$ & Constante de inércia da máquina síncrona dada & \\
& pela relação entre a energia cinética da máquina e & $\mathrm{pu} / \mathrm{pu}$ \\
\hline
\end{tabular}

A Tabela 3.1 define os símbolos utilizados no modelo de máquina síncrona apresentado. Focando a estabilidade a pequenos sinais, construiremos o modelo dinâmico do sistema elétrico a partir da linearização das equações (3.5) a (3.7) em torno de um ponto de equilíbrio estável assim como fora apresentado no Capítulo 2.

Neste sentido, este capítulo visa prover uma explicação de como e porque o movimento mecânico do rotor da máquina síncrona é influenciado por efeitos eletromecânicos assim como examinar como aquele movimento varia dependendo do ponto de operação da máquina síncrona. Introduziremos alguns conceitos importantes no que se refere a estabilidade aliados com sua descrição matemática e explicação física de suas implicações. 


\subsection{Métodos de Construção do Modelo Dinâmico}

\subsubsection{Espaço de Estados}

A representação em espaço de estados, também conhecida como "abordagem no domínio do tempo" fornece uma maneira prática e compacta para modelar e analisar sistemas com múltiplas entradas e saídas. Quando o sistema dinâmico é linear e invariante no tempo, as variáveis são expressas em vetores e as equações diferenciais e algébricas são escritas na forma matricial. O espaço de estados refere-se ao espaço cujos eixos são as variáveis de estado. O estado do sistema pode ser representado como um vetor dentro desse espaço. Trata-se de um método para modelagem, análise e projeto de uma ampla variedade de sistemas.

Um sistema respresentado por espaço de estados é da seguinte forma [7]:

$$
\left\{\begin{array}{l}
\dot{\mathbf{x}}=A \mathbf{x}+B \mathbf{u} \\
\mathbf{y}=C \mathbf{x}+D \mathbf{u}
\end{array}\right.
$$

Em que:

$\mathbf{x} \in \mathbb{R}^{n}$ : é o vetor de estado;

$\mathbf{y} \in \mathbb{R}^{q}$ : é o vetor de saída ou resposta;

$\mathbf{u} \in \mathbb{R}^{m}$ : é o vetor de entrada ou de controle;

$A \in \mathbb{R}^{n \times n}:$ é a matriz de estado;

$B \in \mathbb{R}^{n \times m}$ : é a matriz de entrada ou controle;

$C \in \mathbb{R}^{q \times n}:$ é a matriz de saída;

$D \in \mathbb{R}^{q \times m}:$ é a matriz de controle direto.

Em muitas situações o sinal de entrada de um sistema dinâmico é de natureza periódica. Muitas vezes a análise do comportamento do sistema no tempo não é suficiente para se analisar o problema. Vibrações mecânicas exercidas em uma máquina síncrona devido ao balanceamento inadequado do rotor ou da carga acoplada ao eixo do mesmo são exemplos de sinais de natureza periódica, que em muitos casos apresentam formas de onda muito semelhantes a senóides. Além disso, sinais periódicos, independente de sua natureza, podem ser representados pela soma infinita de harmônicas senoidais. Desta forma, o conhecimento do comportamento do sistema a um sinal de entrada senoidal 
constitui a base para determinação da resposta do sistema para uma larga classe de entradas periódicas. A resposta em frequência pode ser obtida a partir da representação em espaço de estados.

Definição 3.2.1 O método da resposta em frequência é definido como a resposta em regime permanente do sistema quando considerada uma entrada do tipo senoidal. $O$ sinal senoidal constitui o único sinal de entrada e, para um sistema linear, todos os sinais intermediários bem como a saída deste sistema regime permanente também serão senóides. Tais sinais diferem daquele considerado na entrada somente em amplitude e fase [7].

A função de transferência $H(s)=Y(s) / U(s)$ do sistema pode ser obtida a partir da transformada de Laplace da equação que representa o sistema em espaço de estados (3.11). Veja:

$$
\begin{gathered}
s \mathbf{X}=A \mathbf{X}+B \mathbf{U} \\
(s I-A) \mathbf{X}=B \mathbf{U} \\
\mathbf{X}=(s I-A)^{-1} B \mathbf{U}
\end{gathered}
$$

Substituindo (3.14) em (3.11). Temos:

$$
\mathbf{Y}=C(s I-A)^{-1} B \mathbf{U}
$$

Logo a função de transferência $H(s)$ que relaciona a entrada com a saída do sistema é dada por:

$$
H(s)=C(s I-A)^{-1} B
$$

Quando $C^{T}$ e B são matrizes ao invés de vetores, $H(s)$ é dita matriz de transferência. A análise da resposta em frequência pode ser obtida substituindo-se a variável 's' por ' $j \omega$ ' na equação (3.16). Assim:

$$
H(j \omega)=C(j \omega I-A)^{-1} B
$$

Calculando-se numericamente o valor de $H(j \omega)$ para valores discretos de frequência em (3.17), tem-se a resposta em frequência para o sistema.

A representação em espaço de estados acaba por gerar uma matriz de estado A pouco esparsa, ou seja, a maioria de seus elementos não são iguais a zero. Assim, 
o cálculo dos autovalores e dos resíduos da função de transferência, a obtenção dos coeficientes de sensibilidade dos autovalores, a resposta temporal a um degrau unitário e diversas outras funções necessárias para a análise de estabilidade demandariam grande quantidade de tempo e memória computacional quando sistemas de grande porte fossem analisados [8].

\subsubsection{Sistemas Descritores}

Sistemas descritores são caracterizados por um sistema de equações algébrico-diferenciais que assumem a seguinte forma no espaço de estados:

$$
\left\{\begin{array}{l}
E \dot{\mathbf{x}}=A \mathbf{x}(t)+B \mathbf{u}(t) \\
\mathbf{y}(t)=C \mathbf{x}(t)
\end{array}\right.
$$

Em que $\mathbf{x}(\mathrm{t}) \in \mathbb{R}^{n}$ é um vetor de $\mathbf{n}$ variáveis de estados, $\mathbf{u}(\mathrm{t}) \in \mathbb{R}^{m}$ é um vetor de $\mathbf{m}$ entradas, $\mathbf{y}(\mathrm{t}) \in \mathbb{R}^{q}$ um vetor de $\mathbf{q}$ saídas, enquanto que $\mathrm{E} \in \mathbb{R}^{n \times n}, \mathrm{~A} \in \mathbb{R}^{n \times n}$, $\mathrm{B} \in \mathbb{R}^{n \times m}, \mathrm{C} \in \mathbb{R}^{q \times n}$ são representações matriciais das transformações lineares $\mathrm{e}$ invariantes no tempo.

A utilização do sistema de equações linearizadas permite representar o problema da estabilidade eletromecânica do sistema elétrico de potência nos domínios do tempo e da frequência. Nesta representação, em que a matriz Jacobiana aumentada [10] é utilizada, ocorrem um conjunto de equações diferenciais e algébricas linearizadas que podem ser resolvidas simultaneamente. A equação (3.19) mostra como é constituída a matriz de estados A (ou matriz Jacobiana aumentada) por meio de submatrizes.

$$
\left[\begin{array}{c}
\Delta \dot{\mathbf{x}} \\
\mathbf{0}
\end{array}\right]=\left[\begin{array}{ll}
J_{1} & J_{2} \\
J_{3} & J_{4}
\end{array}\right]\left[\begin{array}{c}
\Delta \mathbf{x} \\
\Delta \mathbf{r}
\end{array}\right]+\left[\begin{array}{c}
B_{1} \\
B_{2}
\end{array}\right] \Delta \mathbf{u}
$$

Este modelo possui como característica positiva a facilidade de inclusão de novos dispositivos, sendo desnecessária a representação de uma barra infinita e conseqüentemente não há necessidade de reformulação das equações previamente definidas.

Para a definição destas representações considere um sistema multimáquinas de ng geradores e $n b$. Temos que: 


$$
\begin{array}{r}
\Delta \mathbf{x}=\left[\begin{array}{lllll}
\left(\begin{array}{llllll}
\Delta E_{q 1}^{\prime \prime} & \Delta E_{d 1}^{\prime \prime} & \ldots & \Delta E_{q 1}^{\prime} & \Delta E_{d 1}^{\prime}
\end{array}\right)\left(\begin{array}{llllll}
\Delta E_{q 2}^{\prime \prime} & \Delta E_{d 2}^{\prime \prime} & \ldots & \Delta E_{q 2}^{\prime} & \Delta E_{d 2}^{\prime}
\end{array}\right) \ldots \\
\left(\begin{array}{llllll}
\Delta E_{q n g}^{\prime \prime} & \Delta E_{d n g}^{\prime \prime} & \ldots & \Delta E_{q n g}^{\prime} & \Delta E_{d n g}^{\prime}
\end{array}\right]^{T} \\
\Delta \mathbf{r}=\left[\begin{array}{lllllll}
\left(I_{d 1}\right. & \Delta I_{q 1} & \ldots & \Delta V_{r 1} & \Delta V_{m 1}
\end{array}\right)\left(\begin{array}{lllll}
\Delta I_{d 2} & \Delta I_{q 2} & \ldots & \Delta V_{r 2} & \Delta V_{m 2}
\end{array}\right) \ldots \\
\left(\begin{array}{llllll}
\Delta I_{d n b} & \Delta I_{q n b} & \ldots & \Delta V_{r n b} & \Delta V_{m n b}
\end{array}\right]^{T}
\end{array}\right.
\end{array}
$$

Desta forma, o comportamento dinâmico do sistema elétrico pode ser representado no domínio do tempo conforme a equação (3.19), em que são considerados os vetores de variáveis de estado $(\Delta \mathbf{x})$, de entradas $(\Delta \mathbf{u})$ e variáveis algébricas $(\Delta \mathbf{r})$.

Foi adotada a seguinte ordem para as variáveis de estado e algébricas do sistema:

$$
\begin{array}{r}
E_{q}^{\prime \prime}, E_{d}^{\prime \prime}, E_{q}^{\prime}, E_{d}^{\prime}, \omega, \delta, I_{d}, I_{q}, S_{d}, S_{q}, E_{f d}, P_{m}, V_{P S S}, V_{R E F}, P_{R E F}, V_{r i}, V_{m i}, V_{R E F}^{R T}, V_{t}^{R T}, V_{P S S}^{R T}, X_{1}^{R T}, \\
X_{0005}^{R T}, E_{f d}^{R T}, D \omega^{P S S}, X_{0006}^{P S S}, X_{1}^{P S S}, V_{P S S}^{P S S} .
\end{array}
$$

As variáveis $V_{R E F}^{R T}, V_{t}^{R T}, V_{P S S}^{R T}, X_{1}^{R T}, X_{0005}^{R T}, E_{f d}^{R T}, D \omega^{P S S}, X_{0006}^{P S S}, X_{1}^{P S S}, V_{P S S}^{P S S}$ fazem parte das equações dos controladores a serem implementados no modelo e serão definidas na seção 3.3.3. A matriz de estados $A$ do sistema de potência pode ser obtida pela eliminação do vetor das variáveis algébricas e de entradas na equação (3.19):

$$
\Delta \dot{\mathbf{x}}=\left(J_{1}-J_{2} J_{4}^{-1} J_{3}\right) \Delta \mathbf{x}=A \Delta \mathbf{x}
$$

\subsection{Representação Linear do SEP}

Iniciaremos nesta seção a representação do SEP a partir de suas equações linearizadas em torno do ponto de operação do sistema. O fluxograma representativo deste processo é mostrado na Figura 3.1. 


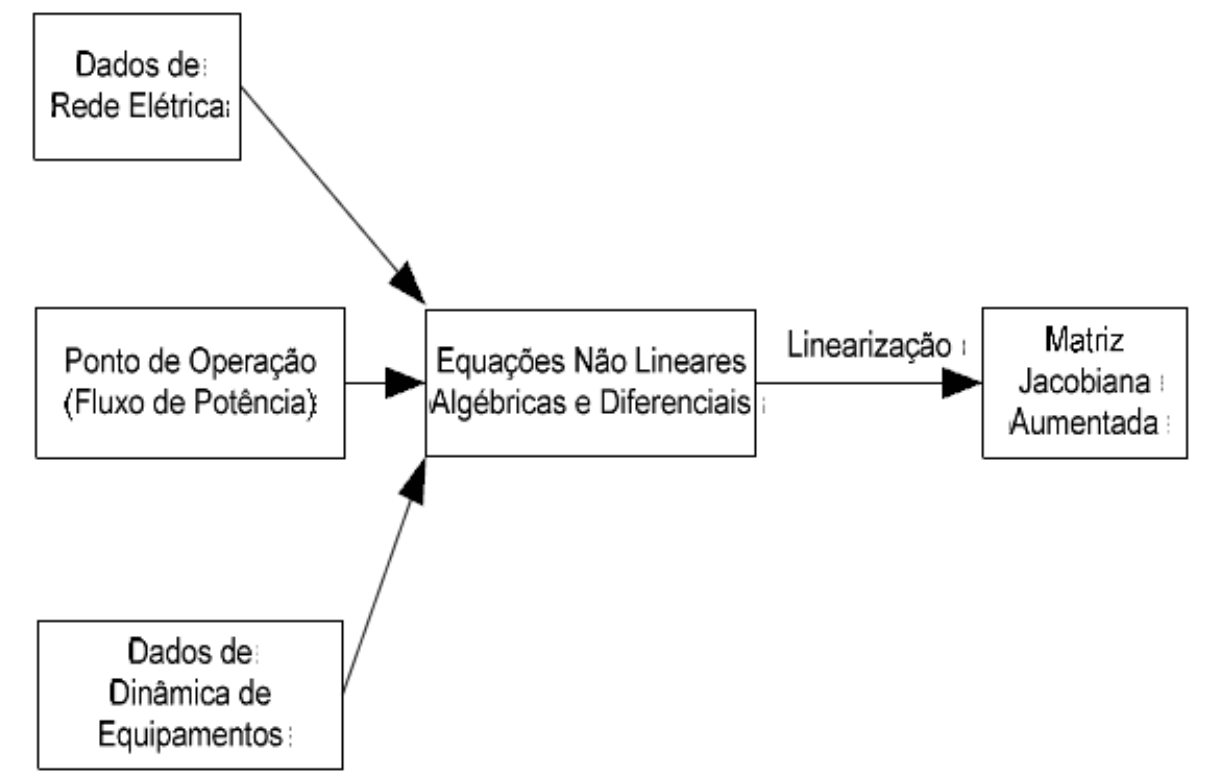

Figura 3.1: Fluxograma para obtenção da representação linear do SEP [6]

Assim, a primeira etapa deste processo é a determinação das condições iniciais das máquinas síncronas.

\subsubsection{Condições Iniciais}

O ângulo inicial $\delta_{i}^{(0)}$ de atuação da máquina síncrona em regime permanente é obtido a partir de:

$$
E_{q i} \angle \delta_{i}^{(0)}=\bar{E}_{q i}=\bar{V}_{t i}+\left(R_{a i}+j X_{q i}\right) \bar{I}_{i}
$$

Conhecemos, a partir dos dados obtidos do fluxo de carga, a tensão $\bar{V}_{t i}$ na barra do gerador. Logo, podemos calcular a corrente inicial entregue pelo gerador. Veja:

$$
\bar{S}_{g i}=P_{g i}+j Q_{g i}
$$

Logo:

$$
\bar{I}_{g i}=\frac{\bar{S}_{g i}^{*}}{\bar{V}_{t i}^{*}}=\frac{P_{g i}-j Q_{g i}}{V_{t i} \angle-\theta_{i}}
$$

Basta realizar a transformação $r-m \rightarrow d-q$ para obtermos os valores iniciais das correntes com as quais iremos trabalhar:

$$
\left[\begin{array}{c}
I_{d i}^{(0)} \\
I_{q i}^{(0)}
\end{array}\right]=\left[\begin{array}{cc}
\sin \delta_{i}^{(0)} & -\cos \delta_{i}^{(0)} \\
\cos \delta_{i}^{(0)} & \sin \delta_{i}^{(0)}
\end{array}\right]\left[\begin{array}{c}
I_{r i}^{(0)} \\
I_{m i}^{(0)}
\end{array}\right]
$$


Considerando o modelo \#6 de gerador adotado pelo PacDyn, a equação de queda de tensão no gerador será:

$$
\left[\begin{array}{c}
V_{d i} \\
V_{q i}
\end{array}\right]=\left[\begin{array}{c}
E_{d i}^{\prime \prime} \\
E_{q i}^{\prime \prime}
\end{array}\right]+\left[\begin{array}{cc}
R_{a i} & -X_{q i}^{\prime \prime} \\
X_{d i}^{\prime \prime} & R_{a i}
\end{array}\right]\left[\begin{array}{c}
I_{d i} \\
I_{q i}
\end{array}\right]
$$

É preciso calcular as condições iniciais a partir do equacionamento:

$$
\left[\begin{array}{c}
V_{d i}^{(0)} \\
V_{q i}^{(0)}
\end{array}\right]=\left[\begin{array}{cc}
\sin \delta_{i}^{(0)} & -\cos \delta_{i}^{(0)} \\
\cos \delta_{i}^{(0)} & \sin \delta_{i}^{(0)}
\end{array}\right]\left[\begin{array}{c}
V_{r i}^{(0)} \\
V_{m i}^{(0)}
\end{array}\right]
$$

E, então, $E_{d i}^{\prime \prime(0)}$ e $E_{q i}^{\prime \prime(0)}$ podem ser calculados. O valor de $E_{q i}^{\prime(0)}$ é obtido a partir da equação 3.20 do modelo $\# 6$ fazendo $\mathrm{SAT}=0$. Ou seja:

$$
E_{q i}^{\prime(0)}-E_{q i}^{\prime \prime(0)}-\left(X_{d i}^{\prime}-X_{d i}^{\prime \prime}\right) I_{d i}^{(0)}=0
$$

O valor de $E_{f d}^{(0)}$ é obtido a partir da equação (3.2) fazendo-se $\frac{d E_{q}^{\prime}}{d t}=0$.

\subsubsection{Equações dos Geradores}

Utilizando-se das equações (3.1) a (3.6) obtem-se as expressões para as quatro primeiras variáveis de estado do sistema em estudo da seguinte forma:

$$
\begin{aligned}
& \Delta \dot{\mathrm{E}}_{d}^{\prime \prime}=\left[\frac{1}{T_{d 0}^{\prime}}\right] \Delta E_{d}^{\prime \prime}+\left[\frac{\left(X_{q}-X_{q}^{\prime \prime}\right)}{T_{q 0}^{\prime \prime}}\right] \Delta I_{q} \\
& \Delta \dot{\mathrm{E}}_{q}^{\prime}=\left[\frac{1}{T_{d 0}^{\prime}}\right] \Delta E_{f d}+\left[\frac{\left(X_{d}-X_{d}^{\prime}\right)}{T_{d 0}^{\prime}\left(X_{d}^{\prime}-X_{l}\right)}\right] \Delta E_{q}^{\prime \prime}-\left[\frac{\left(X_{d}-X_{l}\right)}{T_{d 0}^{\prime}\left(X_{d}^{\prime}-X_{l}\right)}\right] \Delta E_{q}^{\prime}-\left[\frac{\left(X_{d}-X_{d}^{\prime}\right)\left(X_{d}^{\prime \prime}-X_{l}\right)}{T_{d 0}^{\prime}\left(X_{d}^{\prime}-X_{l}\right)}\right] \Delta I_{d} \\
& \Delta \dot{\mathrm{E}}_{q}^{\prime \prime}=\left[\frac{-1}{T_{d 0}^{\prime \prime}}\right] \Delta E_{q}^{\prime \prime}+\left[\frac{1}{T_{d 0}^{\prime \prime}}\right] \Delta E_{q}^{\prime}-\left[\frac{\left(X_{d}^{\prime}-X_{d}^{\prime \prime}\right)}{T_{d 0}^{\prime \prime}}\right] \Delta I_{d}+\left[\frac{\left(X_{d}^{\prime \prime}-X_{l}\right)}{T_{d 0}^{\prime}\left(X_{d}^{\prime}-X_{l}\right)}\right] \Delta \dot{\mathrm{E}}_{q}^{\prime}
\end{aligned}
$$

Substituindo a equação (3.31) em (3.32) temos:

$$
\begin{aligned}
\Delta \dot{\mathrm{E}}_{q}^{\prime \prime}= & {\left[\frac{\left(X_{d}^{\prime \prime}-X_{l}\right)\left(X_{d}-X_{d}^{\prime}\right)}{T_{d 0}^{\prime}\left(X_{d}^{\prime}-X_{l}\right)^{2}}-\frac{1}{T_{d 0}^{\prime \prime}}\right] \Delta E_{q}^{\prime \prime}+\left[\frac{-\left(X_{d}^{\prime \prime}-X_{l}\right)\left(X_{d}-X_{l}\right)}{T_{d 0}^{\prime}\left(X_{d}^{\prime}-X_{l}\right)^{2}}+\frac{1}{T_{d 0}^{\prime \prime}}\right] \Delta E_{q}^{\prime}+} \\
& +\left[\frac{-\left(X_{d}^{\prime \prime}-X_{l}\right)^{2}\left(X_{d}-X_{d}^{\prime}\right)}{T_{d 0}^{\prime}\left(X_{d}^{\prime}-X_{l}\right)^{2}}-\frac{\left(X_{d}^{\prime}-X_{d}^{\prime \prime}\right)}{T_{d 0}^{\prime \prime}}\right] \Delta I_{d}+\left[\frac{\left(X_{d}^{\prime \prime}-X_{l}\right)}{T_{d 0}^{\prime}\left(X_{d}^{\prime}-X_{l}\right)}\right] \Delta E_{f d}
\end{aligned}
$$


Partimos agora para a otenção da expressão para a variação angular $\omega$ correspondente à quinta variável de estado. Ela é obtida a partir das equações (3.4) e (3.5), mais conhecidas como equação swing, do modelo de máquina síncrona em estudo. A sua linearização correspondente é:

$$
\Delta \dot{\omega}=\frac{\Delta P_{m}}{2 H}-\frac{\Delta P_{e}}{2 H}-D_{t} \frac{\Delta \omega}{2 H}
$$

Mas, a potência elétrica é dada por:

$$
P_{e}=R_{a}\left(I_{d}^{2}+I_{q}^{2}\right)+V_{d} I_{d}+V_{q} I_{q}
$$

E, portanto:

$$
\Delta P_{e}=\left[2 R_{a} I_{d 0}+V_{d 0}\right] \Delta I_{d}+\left[2 R_{a} I_{q 0}+V_{q 0}\right] \Delta I_{q}+I_{d 0} \Delta V_{d}+I_{q 0} \Delta V_{q}
$$

Realizando a transformação $r-m \rightarrow d-q$ para obtermos a expressão linearizada de $P_{e}$ em função das variáveis de estado e algébricas utilizadas em nosso modelo, temos:

$$
\left[\begin{array}{c}
\Delta V_{d} \\
\Delta V_{q}
\end{array}\right]=\left[\begin{array}{cc}
\sin \delta_{0} & -\cos \delta_{0} \\
\cos \delta_{0} & \sin \delta_{0}
\end{array}\right]\left[\begin{array}{c}
\Delta V_{r} \\
\Delta V_{m}
\end{array}\right]+\left[\begin{array}{cc}
\cos \delta_{0} & \sin \delta_{0} \\
-\sin \delta_{0} & \cos \delta_{0}
\end{array}\right]\left[\begin{array}{c}
V_{r 0} \\
V_{m 0}
\end{array}\right] \Delta \delta
$$

Como resultado, obtém-se:

$$
\begin{array}{r}
\Delta \dot{\omega}=\frac{\Delta P_{m}}{2 H}-\frac{1}{2 H}\left[I_{d 0}\left(V_{r 0} \cos \delta_{0}+V_{m 0} \sin \delta_{0}\right)+I_{q 0}\left(V_{m 0} \cos \delta_{0}-V_{r 0} \sin \delta_{0}\right)\right] \Delta \delta \\
-\frac{1}{2 H}\left[2 R_{a} I_{d 0}+V_{d 0}\right] \Delta I_{d}-\frac{1}{2 H}\left[2 R_{a} I_{q 0}+V_{q 0}\right] \Delta I_{q}-\frac{1}{2 H}\left[I_{d 0} \sin \delta_{0}+I_{q 0} \cos \delta_{0}\right] \Delta V_{r} \\
-\frac{1}{2 H}\left[I_{q 0} \sin \delta_{0}-I_{d 0} \cos \delta_{0}\right] \Delta V_{m}-\frac{D_{t} \Delta \omega}{2 H}
\end{array}
$$

A expressão para a posição angular $\delta$ do rotor é obtida diretamente da equação swing (3.5). Veja:

$$
\Delta \dot{\delta}=\omega_{0} \Delta \omega
$$

A expressão para as correntes $I_{d}$ e $I_{q}$ no gerador é obtida a partir da equação (3.7) do modelo \#6 que estamos trabalhando. Das equações correspondentes às quedas de tensão no estator, são obtidas:

$0=\left\{\left[V_{m 0} \cos \delta_{0}-V_{r 0} \sin \delta_{0}\right] \Delta \delta+\left[\cos \delta_{0}\right] \Delta V_{r}+\left[\sin \delta_{0}\right] \Delta V_{m}\right\}+[-1] \Delta E_{q}^{\prime \prime}+\left[X_{d}^{\prime \prime}\right] \Delta I_{d}+[R a] \Delta I_{q}$ 
$0=\left\{\left[V_{r 0} \cos \delta_{0}+V_{m 0} \sin \delta_{0}\right] \Delta \delta+\left[\sin \delta_{0}\right] \Delta V_{r}+\left[-\cos \delta_{0}\right] \Delta V_{m}\right\}+[-1] \Delta E_{q}^{\prime \prime}+\left[-X_{d}^{\prime \prime}\right] \Delta I_{d}+[R a] \Delta I_{q}$

Finalmente, são determinadas as expressões que irão definir as tensões nas barras do sistema.

Os circuitos elétricos que representam os sistemas elétricos de potência são baseados na lei dos nós para correntes:

$$
\begin{gathered}
{[\bar{I}]=\left[\bar{Y}_{B U S}\right][\bar{V}]} \\
{[\bar{I}]=\left[\begin{array}{llll}
\bar{I}_{1} & \bar{I}_{2} & \ldots & \bar{I}_{N B}
\end{array}\right]^{T}} \\
{[\bar{V}]=\left[\begin{array}{llll}
\bar{V}_{1} & \bar{V}_{2} & \ldots & \bar{V}_{N B}
\end{array}\right]^{T}} \\
\bar{V}_{i}=V_{i} \angle \theta_{i}=V_{r i}+j V_{m i} \\
\bar{I}_{i}=I_{i} \angle \theta_{i}=I_{r i}+j I_{m i} \\
\bar{Y}_{i j}=G_{i j}+j B_{i j},\left\{\begin{array}{l}
i=1,2, \ldots, N B ; \\
j=1,2, \ldots, N B .
\end{array}\right.
\end{gathered}
$$

Desmembrando a corrente em parte real e imaginária, temos:

$$
\bar{I}_{i}=\sum_{k=1}^{N B} \bar{Y}_{i k} \bar{V}_{k}=\sum_{k=1}^{N B}\left[G_{i k} V_{r k}-B_{i k} V_{m k}\right]+j \sum_{k=1}^{N B}\left[B_{i k} V_{r k}+G_{i k} V_{m k}\right]
$$

Neste caso:

$$
\left[\begin{array}{c}
I_{m i} \\
I_{r i}
\end{array}\right]=\sum_{k=1}^{N B}\left[\begin{array}{cc}
B_{i k} & G_{i k} \\
G_{i k} & -B_{i k}
\end{array}\right]\left[\begin{array}{c}
V_{r k} \\
V_{m k}
\end{array}\right]
$$

No sentido inverso, a corrente pode ser convertida da seguinte forma:

$$
\left[\begin{array}{c}
I_{r i} \\
I_{m i}
\end{array}\right]=\left[\begin{array}{cc}
\sin \delta_{i} & \cos \delta_{i} \\
-\cos \delta_{i} & \sin \delta_{i}
\end{array}\right]\left[\begin{array}{c}
I_{d i} \\
I_{q i}
\end{array}\right]
$$

Desta maneira, a injeção de corrente no sistema é dada pela seguinte equação algébrica: 


$$
\left[\begin{array}{cc}
-\cos \delta_{i} & \sin \delta_{i} \\
\sin \delta_{i} & \cos \delta_{i}
\end{array}\right]\left[\begin{array}{c}
I_{d i} \\
I_{q i}
\end{array}\right]+\sum_{k=1}^{N B}\left[\begin{array}{cc}
-B_{i k} & -G_{i k} \\
-G_{i k} & B_{i k}
\end{array}\right]\left[\begin{array}{c}
V_{r k} \\
V_{m k}
\end{array}\right]=\left[\begin{array}{l}
0 \\
0
\end{array}\right]
$$

Realizando a linearização da expressão (3.51) em torno do ponto de operação inicial, obtem-se:

$$
\left[\begin{array}{cc}
-\cos \delta_{i}^{(0)} & \sin \delta_{i}^{(0)} \\
\sin \delta_{i}^{(0)} & \cos \delta_{i}^{(0)}
\end{array}\right]\left[\begin{array}{c}
\Delta I_{d i} \\
\Delta I_{q i}
\end{array}\right]+\left[\begin{array}{c}
I_{d}^{(0)} \sin \delta_{i}^{(0)}+I_{q}^{(0)} \cos \delta_{i}^{(0)} \\
I_{d}^{(0)} \cos \delta_{i}^{(0)}-I_{q}^{(0)} \sin \delta_{i}^{(0)}
\end{array}\right] \Delta \delta+\sum_{k=1}^{N B}\left[\begin{array}{cc}
-B_{i k} & -G_{i k} \\
-G_{i k} & B_{i k}
\end{array}\right]\left[\begin{array}{c}
\Delta V_{r k} \\
\Delta V_{m k}
\end{array}\right]=\left[\begin{array}{l}
0 \\
0
\end{array}\right]
$$

\subsubsection{Sistema de Excitação}

O desejável aumento da robustez e da flexibilidade dos sistemas elétricos pode tornarse viável com a utilização de dispositivos de eletrônica de potência, que permitem ações de chaveamento e controle de grandezas elétricas, antes limitadas pela atuação de componentes eletromecânicos.

A Figura 3.2 apresenta um diagrama de blocos funcional de um sistema de controle de excitação típico para um gerador síncrono de grande porte. O objetivo dos controladores do tipo Estabilizadores de Sistemas de Potência (PSS's - Power System Stabilizers) é introduzir um torque elétrico em fase com as variações da velocidade e adicionar um sinal suplementar à malha de controle do Regulador Automático de Tensão (RT) do gerador. Dispositivos PSS's possuem como sinais de entrada o desvio da velocidade do rotor, a potência de aceleração, o desvio da frequência, a potência elétrica terminal, dentre outras. Estes estabilizadores são, em geral, constiuídos por circuitos de controle avanço-atraso, que corrigem a fase dos sinais estabilizantes para provocar a modulação da tensão de referência dos reguladores de tensão com o objetivo de introduzir torque de amortecimento aos modos de oscilação local ou interárea. O regulador (de tensão) é o responsável por processar e amplificar os sinais de entrada de controle para um nível e forma adequada para a excitatriz e, ao mesmo tempo, controlar a funções de estabilização e regulação do sistema de excitação [1, 5]. Os demais componentes presentes na Figura 3.2 não serão implementados neste projeto. Um estudo mais aprofundado sobre o assunto pode ser obtido nas referências $[3,5,4,11]$. 


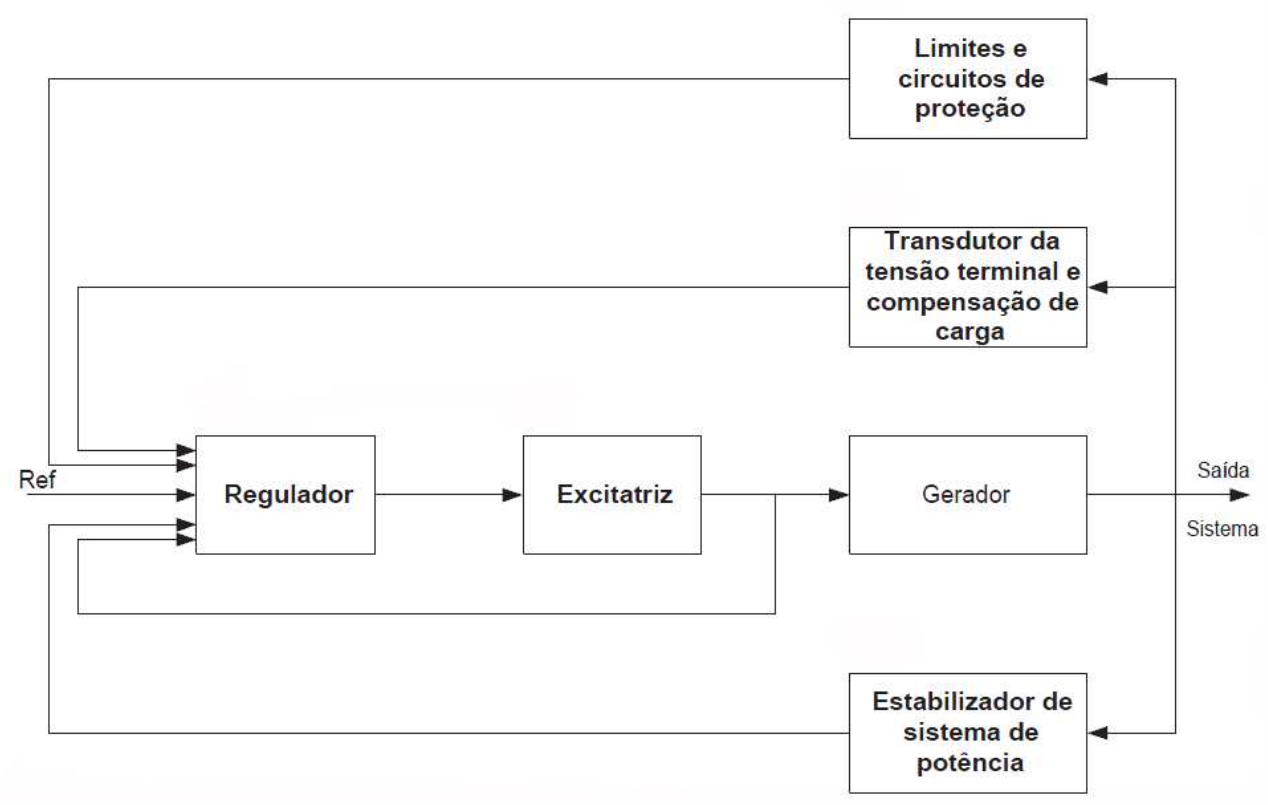

Figura 3.2: Sistema de excitação de um gerador síncrono [1]

A Figura 3.3 mostra um sistema de excitação típico com as respectivas variáveis de entrada e de saída de cada bloco. Já a Figura 3.4 mostra o diagrama de bloco correspondente a blocos de avanço-atraso de fase, utilizado para PSS.

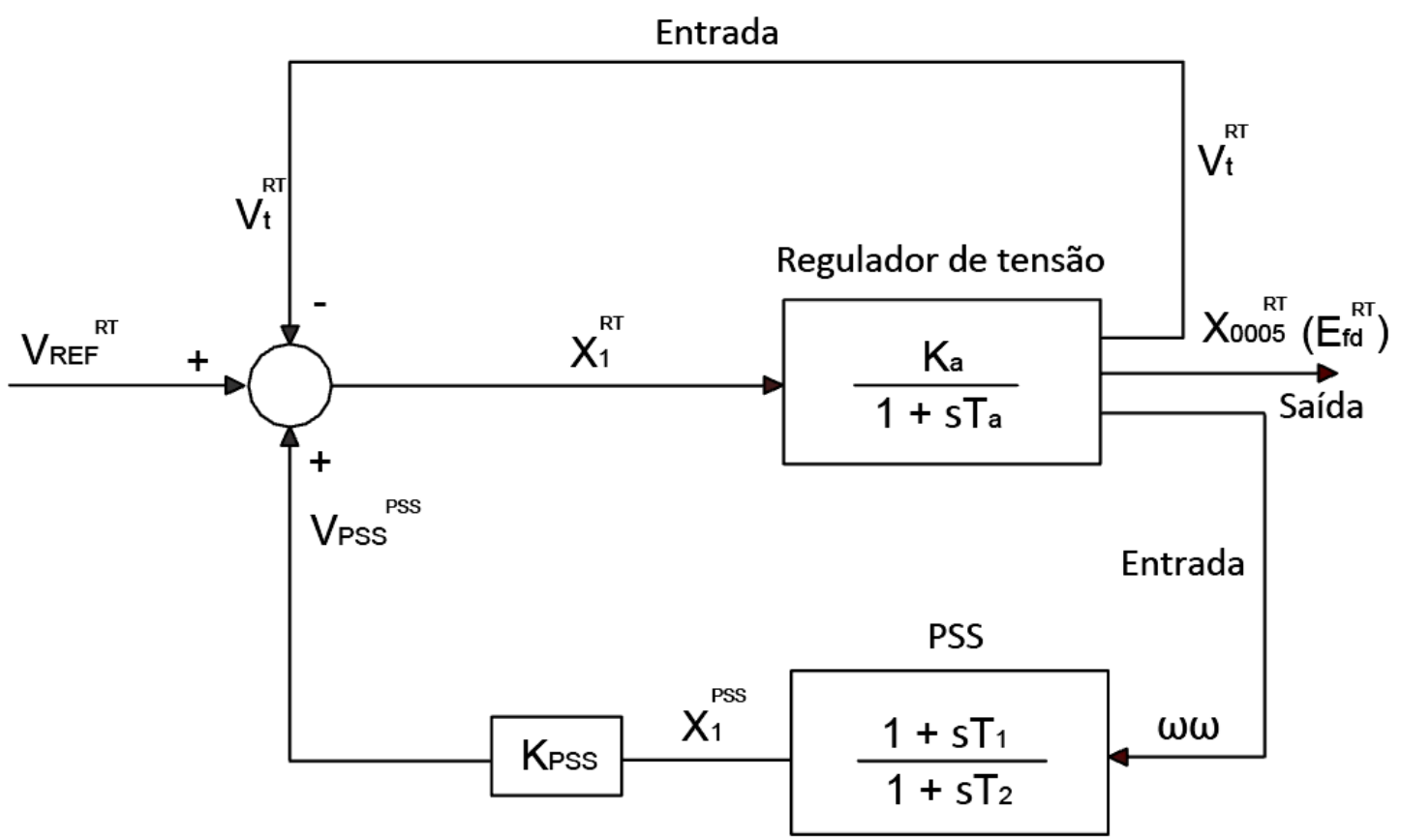

Figura 3.3: Sistema de excitação típico, incluindo sinal adicional produzido pelo PSS 
A Figura 3.5 corresponde a um desmembramento do bloco lead - lag apresentado na Figura 3.4. Note-se que em cada diagrama de bloco são incluídas variáveis que servirão como dados entrada/saída para uso nos códigos dos programas.

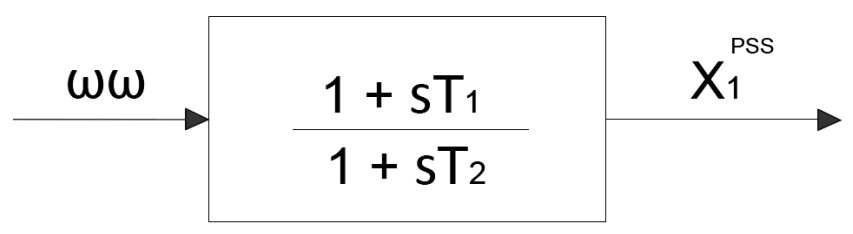

Figura 3.4: Bloco lead - lag utilizado como função de transferência do PSS (não está sendo considerado o bloco washout nesta modelagem)

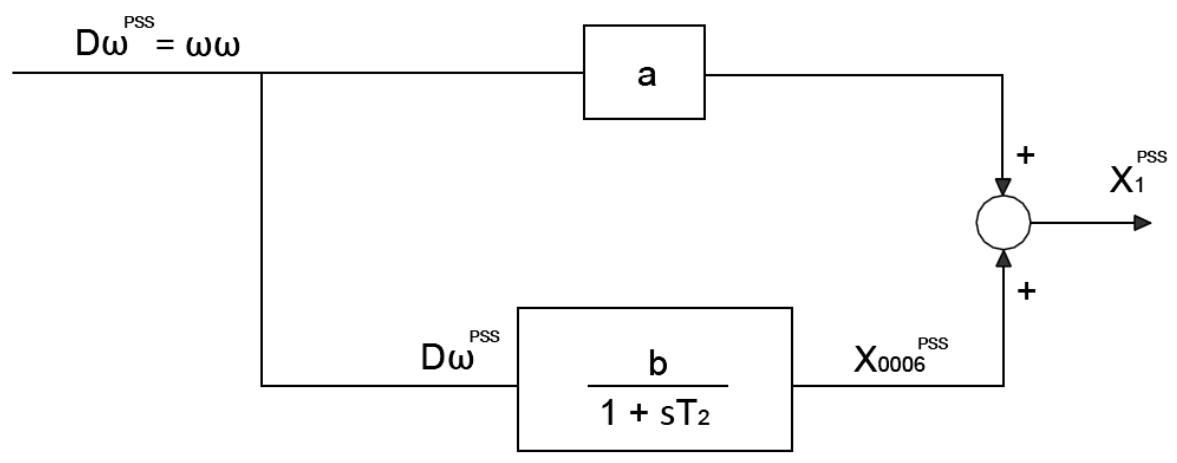

Figura 3.5: Modelo do PSS com o bloco lead - lag desmembrado em um ganho e um bloco de atraso de primeira ordem

Em que: $a=\frac{T_{1}}{T_{2}}$ e $b=\left(1-\frac{T_{1}}{T_{2}}\right)$.

Realizando as devidas manipulações algébricas e passando para o domínio do tempo, obtém-se o modelo linear do controlador PSS:

$0=\omega-D \omega^{P S S}$

$\dot{X}_{0006}^{P S S}=\left(\frac{T_{2}-T_{1}}{T_{2}^{2}}\right) D \omega^{P S S}+\left(\frac{-1}{T_{2}}\right) X_{0006}^{P S S}$

$0=\left(\frac{T_{1}}{T_{2}}\right) D \omega^{P S S}+X_{0006}^{P S S}-X_{1}^{P S S}$

$0=K_{P S S} X_{1}^{P S S}-V_{P S S}^{P S S}$

Optou-se por criar variáveis redundantes, como aquela presente na equação (3.53) com o intuito de facilitar a inserção/retirada dos dispositivos do sistema de excitação, em qualquer um dos geradores em estudo. Desta maneira, não há mistura de variáveis de geradores com as dos controladores e aqueles podem ser analisados separadamente. 
Partindo agora para a obtenção das equações do regulador de tensão mostrado na Figura 3.3, obtemos:

$$
\frac{X_{0005}^{R T}}{\left(X_{1}^{R T}\right) s}=\frac{K_{a}}{1+s T_{a}}
$$

Realizando as devidas linearizações e manipulações algébricas, obtém-se no domínio do tempo:

$0=V_{R E F}-V_{R E F}^{R T}$

$0=-V_{t}^{R T}+\frac{V_{r i}^{(0)}}{\left|V_{t i}^{(0)}\right|} \Delta V_{r i}+\frac{V_{m i}^{(0)}}{\left|V_{t i}^{(0)}\right|} \Delta V_{m i}$

$0=V_{P S S}-V_{P S S}^{R T}$

$0=V_{R E F}^{R T}-V_{t}^{R T}+V_{P S S}^{R T}-X_{1}^{R T}$

$\dot{E}_{f d}^{R T}=\left(\frac{K_{a}}{T_{a}}\right) X_{1}^{R T}+\left(\frac{-1}{T_{a}}\right) X_{0005}^{R T}$

$0=X_{0005}^{R T}-E_{f d}^{R T}$

\subsection{Métodos de Análise do Modelo Dinâmico}

\subsubsection{Autovalores, Autovetores e Transformações}

O cálculo dos autovalores e seus autovetores associados da matriz de estados $A$ permite caracterizar a estabilidade local de um determinado ponto de operação do sistema. Esta é uma técnica também conhecida como análise modal. Esta seção busca apresentar os conceitos básicos utilizados na análise de estabilidade de sistemas de potência. Um estudo mais aprofundado pode ser encontrado nas referências $[1,3,4]$.

\section{Autovalores}

Os autovalores da matriz $A$ são dados pelos valores dos parâmetro escalar $\lambda$ para o qual existem soluções não triviais para a seguinte equação:

$$
A \mathbf{x}=\lambda \mathbf{x}
$$


Em que:

$\diamond$ A é uma matriz $n \times n$ (real para sistemas físicos tal como um sistema de potência);

$\diamond \mathrm{x}$ é um vetor $n \times 1$.

Para encontrarmos os autovalores, a equação (3.64) pode ser escrita na forma

$$
(A-\lambda I) \mathbf{x}=0
$$

de solução não trivial

$$
\operatorname{det}(A-\lambda I)=0
$$

A expansão de (3.66) fornece a equacão característica, da qual as $n$ soluções

$$
\lambda=\lambda_{1}, \lambda_{2}, \ldots, \lambda_{n}
$$

são os autovalores da matriz $A$. Esses autovalores podem ser reais ou complexos. Se $A$ for real, autovalores complexos ocorrem sempre em pares conjugados. A dependência no tempo de um modo correspondente a um autovalor $\lambda_{i}$ é dada por $e^{\lambda_{i t}}$. Assim, a estabilidade do sistema é determinada pelos autovalores da matriz A da seguinte forma:

1. Um autovalor real corresponde à um modo não oscilatório. Um autovalor real negativo corresponde a um modo estável e um autovalor real positivo caracteriza instabilidade monotônica.

2. Autovalores complexos ocorrem em pares conjugados, e cada par corresponde a um modo oscilatório. A parte real componente desses autovalores fornece o amortecimento, enquanto que a parte imaginária fornece a frequência de oscilação. Se a parte real for negativa as oscilações são amortecidas, caso contrário (parte real positiva), as oscilações tem amplitude crescente caracterizando uma instabilidade oscilatória.

Para um par de autovalores complexo conjugado:

$$
\lambda=\sigma \pm j \omega_{d}
$$


a frequência de oscilação do modo $e^{\lambda t} \mathrm{em} \mathrm{Hz}$ é dada por:

$$
f=\frac{\omega_{d}}{2 \pi}
$$

e a taxa de amortecimento por:

$$
\zeta=\frac{-\sigma}{\sqrt{\sigma^{2}+\omega_{d}^{2}}}
$$

\section{Autovetores}

A partir do cálculo dos autovalores da matriz $A$ e dos autovetores à direita e à esquerda, é também possível determinar fatores de participação das variáveis de estado nos modos críticos do sistema [1]. Esses fatores são importantes para identificação das possíveis causas de problemas relacionados com modos fracamente amortecidos ou instáveis. O fator de participação é definido como um produto, elemento por elemento, dos autovetores à direita e à esquerda da matriz $A$. Se $\lambda_{i}$ é o i-ésimo autovalor de $A$, e $\phi_{i}, \psi_{i}$ e são, respectivamente, os autovetores à direita e à esquerda associados a $\lambda_{i}, \mathrm{o}$ fator de participação (FP) da variável de estado $n$, referente ao modo $i$ é definido por:

$$
F P_{n i}=\phi_{i n} \psi_{i n}
$$

Os fatores de participação são quantidades adimensionais que revelam quais geradores estão associados a cada modo eletromecânico de um sistema de potência. Eles indicam, portanto, onde devem ser tomadas medidas de reforço, tais como a instalação de PSSs ou de controladores FACTS, para se alcançar o adequado amortecimento de oscilações eletromecânicas de baixa frequência. 


\subsubsection{Critério de Estabilidade de Nyquist}

O critério de Nyquist permite a avaliação da estabilidade de malha fechada de um sistema com realimentação a partir do conhecimento dos pólos e do gráfico da resposta em frequência da função de transferência de malha aberta [7]. Nesta seção, iremos realizar uma apresentação básica a respeito do critério de Nyquist.

Considere o sistema mostrado na Figura 3.6. A função de transferência, em cascata, do controlador e do processo é dada por $G(s)$ e a do elemento sensor é dada por $H(s)$. A função de transferência deste sistema em malha aberta é dada por $G(s) H(s)$ e a de malha fechada é dada por:

$$
M(s)=\frac{G(s)}{1+G(s) H(s)}
$$

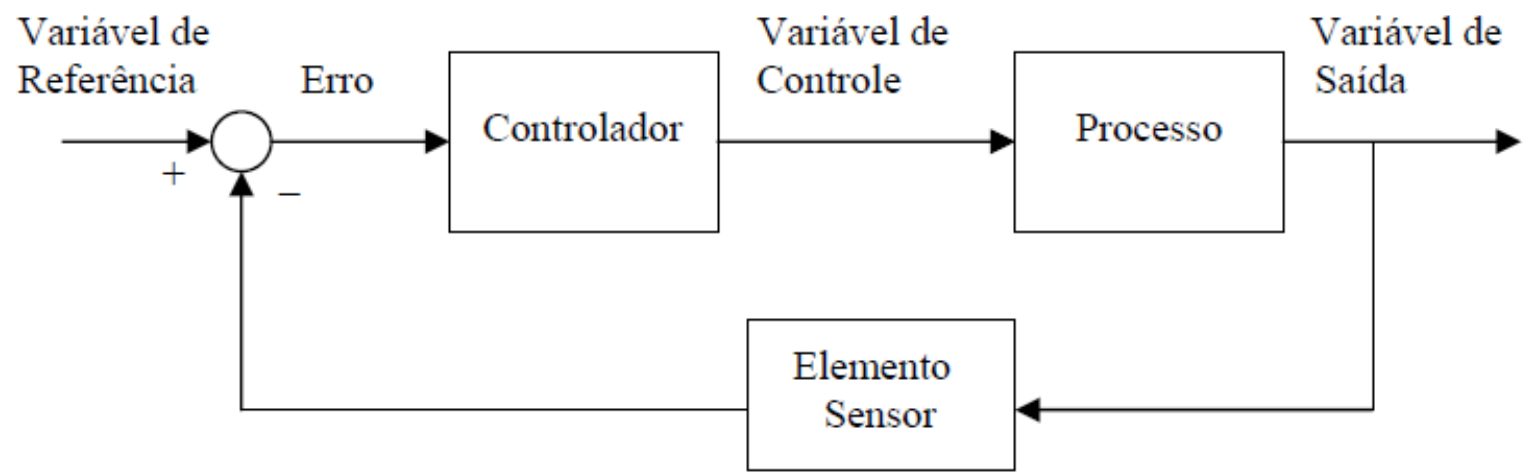

Figura 3.6: Representação em diagrama de blocos de um sistema de controle realimentado

Baseado no princípio do argumento, o critério de Nyquist estabelece que:

$$
N=Z-P
$$

em que $\mathrm{N}$ é o número de circunscrições do ponto $(-1, j 0)$ no sentindo horário feitas pelo gráfico da resposta em frequência de $\mathrm{G}(\mathrm{s}) \mathrm{H}(\mathrm{s})$ quando a frequência varia de $-\infty$ a $+\infty$. P é o número de pólos (ou autovalores) instáveis de $\mathrm{G}(\mathrm{s}) \mathrm{H}(\mathrm{s})$ e $\mathrm{Z}$ é o número de zeros de $1+\mathrm{G}(\mathrm{s}) \mathrm{H}(\mathrm{s})$ no semiplano direito do plano complexo. Observe que os zeros de $1+\mathrm{G}(\mathrm{s}) \mathrm{H}(\mathrm{s})$ são os pólos da função de transferência em malha fechada dada por (3.71).

Para um sistema estável em malha fechada, temos que $Z=0$. Assim, de acordo com (3.72), um sistema em malha fechada é estável se o número de circunscrições do ponto 
$(-1, j 0)$ no sentido anti-horário feitas pelo gráfico de Nyquist de $G(s) H(s)$ for igual ao número de pólos instáveis de $G(s) H(s)$, isto é, deve-se ter $N=-P$. O gráfico de Nyquist pode ser obtido somente para valores positivos da frequência, nesse caso o número de circunscrições que garante a estabilidade do sistema em malha fechada é dado por $N / 2$.

Nas técnicas clássicas de controle, a estabilidade de um sistema é avaliada através dos conceitos de margens de fase e de ganho. Assim, o estabilizador é sempre projetado de forma a propiciar boas margens de fase e de ganho ao sistema. O estabilizador PSS do gerador também pode ser projetado através do gráfico de Nyquist. A função de transferência do sistema é representada por $G(s)$, enquanto $H(s)$ é a função de transferência do controlador PSS a ser projetado. 


\section{Capítulo 4 MODELAGEM DE CARGAS}

\subsection{Contextualização}

A operação estável de um sistema de potência depende da capacidade de continuar a corresponder a saída elétrica das unidades geradoras com a carga elétrica no sistema. A modelagem de cargas é complicada, porque uma barra de carga típica representada em estudos de estabilidade é composta por um grande número de dispositivos, tais como lâmpadas fluorescentes e incandescentes, geladeiras, aquecedores, compressores, motores, fornos, etc. A composição exata da carga é difícil de estimar. Além disso, as mudanças na composição dependem de vários fatores, incluindo o tempo (hora, dia, estação do ano), as condições meteorológicas e o estado da economia. Mesmo se a composição de carga fosse exatamente conhecida, seria impraticável representar cada componente individualmente, pois normalmente há milhões desses componentes na carga total fornecida por um sistema de potência. Portanto, a representação de carga em estudos de sistema é baseado em uma quantidade considerável de simplificação [1, 3].

Iremos discutir, neste capítulo, os conceitos básicos de modelagem de carga, a composição de carga e características de componentes, e de aquisição dos parâmetros do modelo de carga.

\subsection{Conceitos Básicos de Modelagem de Carga}

Em estabilidade de sistemas de potência e de estudos de fluxos de potência, é comum representar as características de carga composta visto de pontos de entrega. A figura 4.1 a seguir representa a carga agregada em uma subestação de transmissão (Barra A) que inclui geralmente, além dos dispositivos ligados a barra, os efeitos da subestação de transformadores abaixadores, alimentadores de sub-transmissão, alimentadores de distribuição, transformadores de distribuição, reguladores de tensão, e dispositivos de 
compensação de potencia reativa $[1,3]$. Os modelos de carga são tradicionalmente classificados em duas categorias: modelo estático e modelo dinâmico.

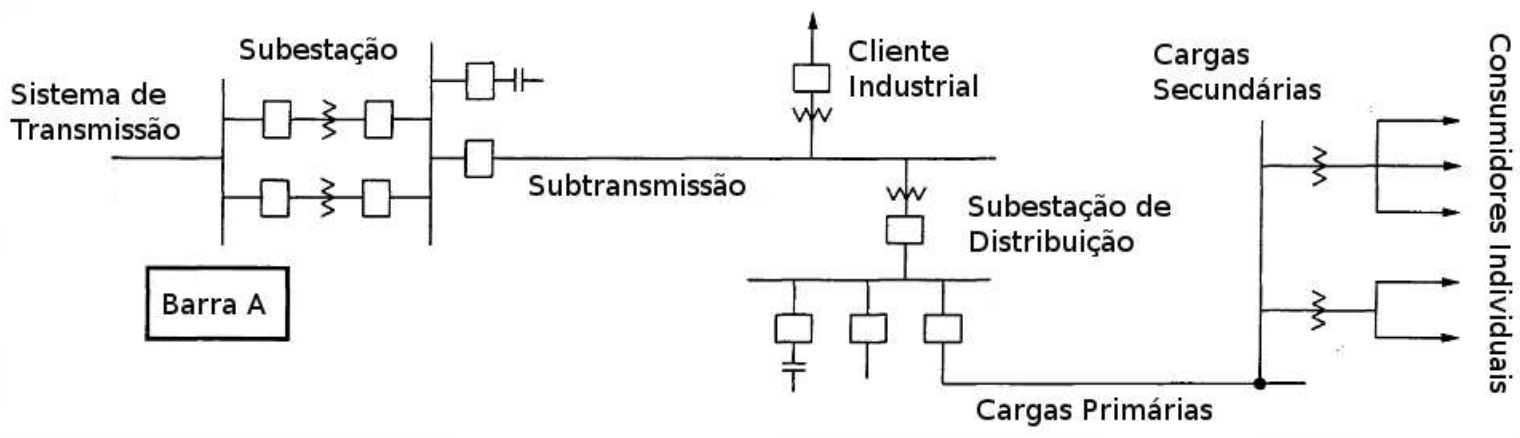

Figura 4.1: Configuração do Sistema Elétrico de Potência enfatizando as cargas presentes no sistema a partir da barra de carga A [1]

\section{Modelo de Carga Estática Não-Linear}

Um modelo de carga estática expressa as características da carga, a qualquer instante do tempo como funções algébricas da magnitude da frequência e tensão na barra em um dado instante. O componente de potência ativa $P$ e de potência reativa $Q$ são considerados separadamente $[4,3]$.

Tradicionalmente, a carga varia exponencialmente com a tensão na barra. Este é o modelo exponencial:

$$
\begin{aligned}
& P_{c}=P_{c}^{(0)}(\bar{V})^{a} \\
& Q_{c}=Q_{c}^{(0)}(\bar{V})^{b}
\end{aligned}
$$

Em que,

$$
\bar{V}=\frac{V}{V^{(0)}}
$$

O índice 0 identifica os valores das respectivas variáveis na condição de operação inicial. 
Os parâmetros deste modelo são os expoentes $a$ e $b$. Com estes expoentes igual a 0,1 ou 2, o modelo representa as cargas em potência, corrente ou impedância constantes, respectivamente. Para cargas compostas, seus valores dependem das características de agregação de componentes de carga [1].

O expoente de $a$ (ou $b$ ) é quase igual à inclinação $d P / d V$ (ou $d Q / d V$ ) com $V=V_{0}$. Para sistemas de cargas composto, o expoente $a$ varia geralmente entre 0,5 e 1,8, já o $b$ é tipicamente entre 1,5 e 6.

Um modelo alternativo amplamente usado para representar a dependência da tensão de cargas é o modelo polinomial:

$$
\begin{aligned}
& P_{c}=P_{c}^{(0)}\left[(1-a-b)+a \frac{\left|V_{i}\right|}{\left|V_{i}^{(0)}\right|}+b \frac{\left|V_{i}\right|^{2}}{\left|V_{i}^{(0)}\right|^{2}}\right] \\
& Q_{c}=Q_{c}^{(0)}\left[(1-c-d)+c \frac{\left|V_{i}\right|}{\left|V_{i}^{(0)}\right|}+d \frac{\left|V_{i}\right|^{2}}{\left|V_{i}^{(0)}\right|^{2}}\right]
\end{aligned}
$$

Este modelo é comumente denominado como modelo ZIP, pois é composto de impedância constante $(Z)$, corrente constante $(I)$, e potência constante $(P)$. Os parâmetros do modelo são os coeficientes $a, b, c$ e $d$, que definem a proporção de cada componente [3].

\section{Modelo de Carga Dinâmica}

A resposta das cargas para mudanças de tensão e de freqüência é rápido, e o estado estacionário da resposta é alcançado muito rapidamente. Isto é verdade pelo menos para pequenas mudanças de amplitude tensão/freqüência.

Há, no entanto, muitos casos em que é necessário utilizar o modelo dinâmico de cargas. Estudos de oscilações interárea, estabilidade de tensão e estabilidade a longo termo muitas vezes requer a modelagem dinâmica. Neste trabalho, entretanto, estas situações 
não foram consideradas. Ao invés disso será considerada a modelagem estática de cargas.

\subsection{Modelagem de Cargas Estáticas Não-Lineares}

Considere a situação genérica em que a carga composta por $P_{c}$ e $Q_{c}$ é conectada ao barramento $i$, conforme indicado na Figura 4.2.

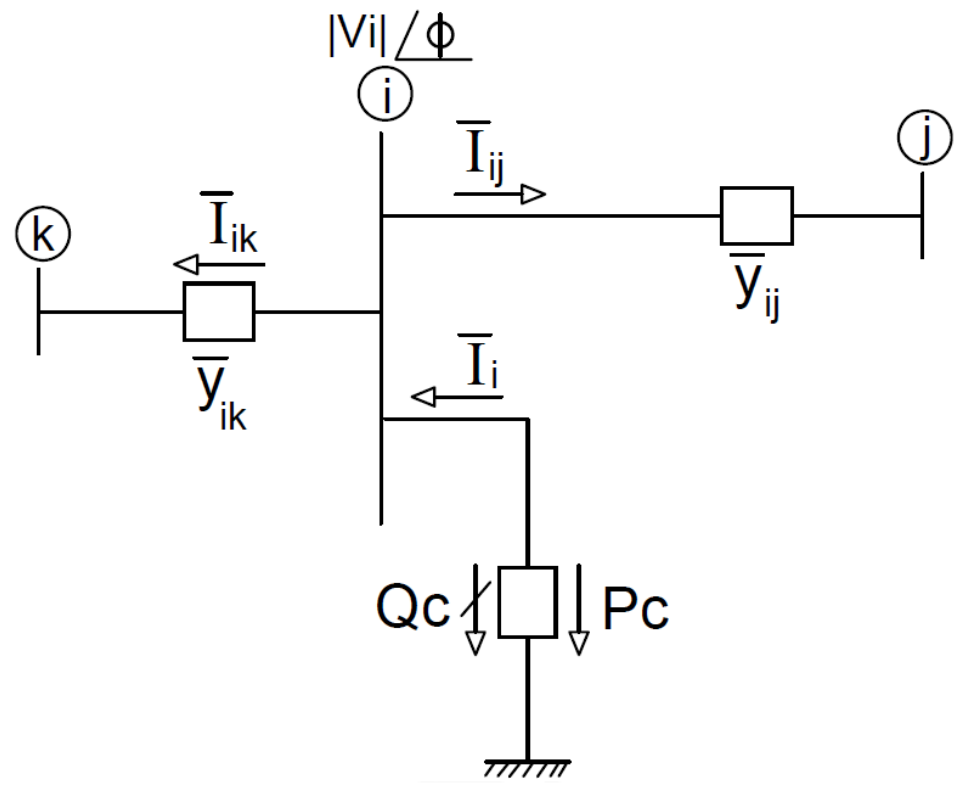

Figura 4.2: Injeção de corrente na barra de carga

Na Figura 4.2, assume-se que $P_{c}$ e $Q_{c}$ são números reais positivos, indicando que a carga absorve esses tipos de potência. No caso de $Q_{c}$ positivo, significa ainda que a potência reativa é indutiva. Então a corrente indicada na barra é:

$$
\bar{I}_{i}=\bar{y}_{i j}\left(\bar{V}_{i}-\bar{V}_{j}\right)+\bar{y}_{i k}\left(\bar{V}_{i}-\bar{V}_{k}\right)
$$

Sendo que,

$$
\bar{S}_{c i}=\bar{V}_{i} \bar{I}_{i}^{*}=-\left[P_{c i}+j Q_{c i}\right]
$$


Reorganizando a equação da corrente:

$$
\begin{gathered}
\bar{I}_{i}=\left[\bar{y}_{i j}+\bar{y}_{i k}\right] \bar{V}_{i}+\left[-\bar{y}_{i j}\right] \bar{V}_{j}+\left[-\bar{y}_{i k}\right] \bar{V}_{k} \\
\bar{I}_{i}=\bar{Y}_{i i} \bar{V}_{i}+\bar{Y}_{i j} \bar{V}_{j}+\bar{Y}_{i k} \bar{V}_{k}
\end{gathered}
$$

Realizando a linerarização:

$$
\Delta \bar{I}_{i}=Y_{i i} \Delta \bar{V}_{i}+Y_{i j} \Delta \bar{V}_{j}+Y_{i k} \Delta \bar{V}_{k}
$$

Matricialmente, as equações são rearranjadas de modo que se trabalhe com o vetor de corrente $\left[\begin{array}{ll}\Delta I_{m i} & \Delta I_{r i}\end{array}\right]^{T}$. Assim:

$$
-\left[\begin{array}{c}
\Delta I_{m i} \\
\Delta I_{r i}
\end{array}\right]=\left[\begin{array}{cc}
-B_{i i} & -G_{i i} \\
-G_{i i} & B_{i i}
\end{array}\right]\left[\begin{array}{c}
\Delta V_{r i} \\
V_{m i}
\end{array}\right]+\left[\begin{array}{cc}
-B_{i j} & -G_{i j} \\
-G_{i j} & B_{i j}
\end{array}\right]\left[\begin{array}{c}
\Delta V_{r j} \\
V_{m j}
\end{array}\right]+\left[\begin{array}{cc}
-B_{i k} & -G_{i k} \\
-G_{i k} & B_{i k}
\end{array}\right]\left[\begin{array}{c}
\Delta V_{r k} \\
V_{m k}
\end{array}\right]
$$

Calculando $\Delta I_{m i}$ e $\Delta I_{r i}$ :

$$
\left[V_{r i}+j V_{m i}\right]\left[I_{r i}-j I_{m i}\right]=-\left[P_{c i}\left(V_{r i}, V_{m i}\right)+j Q_{c i}\left(V_{r i}, V_{m i}\right)\right]
$$

Separando em parte real e imaginária:

$$
\begin{gathered}
\left(V_{r i} I_{r i}+V_{m i} I_{m i}\right)+j\left(V_{m i} I_{r i}-V_{r i} I_{m i}\right)=-P_{c i}-j Q_{c i} \\
\left\{\begin{array}{l}
V_{r i} I_{r i}+V_{m i} I_{m i}=-P_{c i}\left(V_{r i}, V_{m i}\right) \\
V_{m i} I_{r i}-V_{r i} I_{m i}=-Q_{c i}\left(V_{r i}, V_{m i}\right)
\end{array}\right.
\end{gathered}
$$

Linearizando a parte real da equação (4.13):

$$
V_{r i}{ }^{(0)} \Delta I_{r i}+I_{r i}{ }^{(0)} \Delta V_{r i}+V_{m i}{ }^{(0)} \Delta I_{m i}+I_{m i}{ }^{(0)} \Delta V_{m i}=-\frac{\partial P_{c i}}{\partial V_{r i}} \Delta V_{r i}-\frac{\partial P_{c i}}{\partial V_{m i}} \Delta V_{m i}
$$

Reorganizando a equeação (4.15) temos, então, a sequinte expressão:

$$
\left[\begin{array}{ll}
V_{m i}{ }^{(0)} & V_{r i}{ }^{(0)}
\end{array}\right]\left[\begin{array}{c}
\Delta I_{m i} \\
\Delta I_{r i}
\end{array}\right]=-\left[\begin{array}{ll}
\left(I_{r i}{ }^{(0)}+\frac{\partial P_{c i}}{\partial V_{r i}}\right) & \left(I_{m i}{ }^{(0)}+\frac{\partial P_{c i}}{\partial V_{m i}}\right)
\end{array}\right]\left[\begin{array}{c}
\Delta V_{r i} \\
\Delta V_{m i}
\end{array}\right]
$$


Linearizando agora a parte imaginária da equação (4.13):

$$
V_{m i}{ }^{(0)} \Delta I_{r i}+I_{r i}{ }^{(0)} \Delta V_{m i}-V_{r i}{ }^{(0)} \Delta I_{m i}-I_{m i}{ }^{(0)} \Delta V_{r i}=-\frac{\partial Q_{c i}}{\partial V_{r i}} \Delta V_{r i}-\frac{\partial Q_{c i}}{\partial V_{m i}} \Delta V_{m i}
$$

Reorganizando a equação (4.17), temos:

$$
\left[\begin{array}{ll}
-V_{r i}{ }^{(0)} & V_{m i}{ }^{(0)}
\end{array}\right]\left[\begin{array}{c}
\Delta I_{m i} \\
\Delta I_{r i}
\end{array}\right]=-\left[\begin{array}{ll}
\left(-I_{m i}{ }^{(0)}+\frac{\partial Q_{c i}}{\partial V_{r i}}\right) & \left(I_{r i}{ }^{(0)}+\frac{\partial Q_{c i}}{\partial V_{m i}}\right)
\end{array}\right]\left[\begin{array}{c}
\Delta V_{r i} \\
\Delta V_{m i}
\end{array}\right]
$$

Finalmente, montamos a matriz da equação (4.14):

$$
\left[\begin{array}{cc}
V_{m i}{ }^{(0)} & V_{r i}{ }^{(0)} \\
-V_{r i}{ }^{(0)} & V_{m i}{ }^{(0)}
\end{array}\right]\left[\begin{array}{c}
\Delta I_{m i} \\
\Delta I_{r i}
\end{array}\right]=-\left[\begin{array}{cc}
\left(I_{r i}{ }^{(0)}+\frac{\partial P_{c i}}{\partial V_{r i}}\right) & \left(I_{m i}{ }^{(0)}+\frac{\partial P_{c i}}{\partial V_{m i}}\right) \\
\left(-I_{m i}{ }^{(0)}+\frac{\partial Q_{c i}}{\partial V_{r i}}\right) & \left(I_{r i}{ }^{(0)}+\frac{\partial Q_{c i}}{\partial V_{m i}}\right)
\end{array}\right]\left[\begin{array}{c}
\Delta V_{r i} \\
\Delta V_{m i}
\end{array}\right]
$$

Para simplificação dos cálculos, define-se $T_{i}$

$$
T_{i}=\left[\begin{array}{cc}
V_{m i}{ }^{(0)} & V_{r i}{ }^{(0)} \\
-V_{r i}{ }^{(0)} & V_{m i}{ }^{(0)}
\end{array}\right]
$$

Isolando a matriz das correntes $\Delta I_{m i}$ e $\Delta I_{r i}$ :

$$
\left[\begin{array}{c}
\Delta I_{m i} \\
\Delta I_{r i}
\end{array}\right]=-T_{i}^{-1}\left[\begin{array}{cc}
\left(I_{r i}{ }^{(0)}+\frac{\partial P_{c i}}{\partial V_{r i}}\right) & \left(I_{m i}{ }^{(0)}+\frac{\partial P_{c i}}{\partial V_{m i}}\right) \\
\left(-I_{m i}{ }^{(0)}+\frac{\partial Q_{c i}}{\partial V_{r i}}\right) & \left(I_{r i}{ }^{(0)}+\frac{\partial Q_{c i}}{\partial V_{m i}}\right)
\end{array}\right]\left[\begin{array}{c}
\Delta V_{r i} \\
\Delta V_{m i}
\end{array}\right]
$$

Adota-se para a simplificação de cálculos:

$$
T_{i}^{-1}\left[\begin{array}{cc}
\left(I_{r i}{ }^{(0)}+\frac{\partial P_{c i}}{\partial V_{r i}}\right) & \left(I_{m i}{ }^{(0)}+\frac{\partial P_{c i}}{\partial V_{m i}}\right) \\
\left(-I_{m i}{ }^{(0)}+\frac{\partial Q_{c i}}{\partial V_{r i}}\right) & \left(I_{r i}{ }^{(0)}+\frac{\partial Q_{c i}}{\partial V_{m i}}\right)
\end{array}\right]=\left[\begin{array}{ll}
\alpha_{i} & \beta_{i} \\
\gamma_{i} & \sigma_{i}
\end{array}\right]
$$

Após a simplificação, uma nova expressão para a equação (4.21) é dada por:

$$
\left[\begin{array}{c}
\Delta I_{m i} \\
\Delta I_{r i}
\end{array}\right]=-\left[\begin{array}{cc}
\alpha_{i} & \beta_{i} \\
\gamma_{i} & \sigma_{i}
\end{array}\right]\left[\begin{array}{c}
\Delta V_{r i} \\
\Delta V_{m i}
\end{array}\right]
$$


Igualando a equação (4.23) e a equação (4.11), temos:

$$
\begin{aligned}
& {\left[\begin{array}{cc}
\alpha_{i} & \beta_{i} \\
\gamma_{i} & \sigma_{i}
\end{array}\right]\left[\begin{array}{c}
\Delta V_{r i} \\
\Delta V_{m i}
\end{array}\right]=\left[\begin{array}{cc}
-B_{i i} & -G_{i i} \\
-G_{i i} & B_{i i}
\end{array}\right]\left[\begin{array}{c}
\Delta V_{r i} \\
V_{m i}
\end{array}\right]+\left[\begin{array}{cc}
-B_{i j} & -G_{i j} \\
-G_{i j} & B_{i j}
\end{array}\right]\left[\begin{array}{c}
\Delta V_{r j} \\
V_{m j}
\end{array}\right]+\left[\begin{array}{cc}
-B_{i k} & -G_{i k} \\
-G_{i k} & B_{i k}
\end{array}\right]\left[\begin{array}{c}
\Delta V_{r k} \\
V_{m k}
\end{array}\right]} \\
& {\left[\begin{array}{l}
0 \\
0
\end{array}\right]=\left[\begin{array}{cc}
-\left(B_{i i}+\alpha_{i}\right) & -\left(G_{i i}+\beta_{i}\right) \\
-\left(G_{i i}+\gamma_{i}\right) & \left(B_{i i}-\sigma_{i}\right)
\end{array}\right]\left[\begin{array}{c}
\Delta V_{r i} \\
V_{m i}
\end{array}\right]+\left[\begin{array}{cc}
-B_{i j} & -G_{i j} \\
-G_{i j} & B_{i j}
\end{array}\right]\left[\begin{array}{c}
\Delta V_{r j} \\
V_{m j}
\end{array}\right]+\left[\begin{array}{cc}
-B_{i k} & -G_{i k} \\
-G_{i k} & B_{i k}
\end{array}\right]\left[\begin{array}{c}
\Delta V_{r k} \\
V_{m k}
\end{array}\right]}
\end{aligned}
$$

Neste ponto, observa-se que a modelagem de cargas irá influenciar apenas os elementos própios da matriz $Y_{B U S}$ formada pela resolução do fluxo de carga, ou seja, $Y_{B U S_{i i}}$.

Determinando as derivadas presentes na equação (4.21):

$$
\frac{\partial P_{c i}}{\partial V_{r i}}=P_{c i}^{(0)}\left[a_{i} \frac{\partial\left|V_{i}\right|}{\partial V_{r i}} \cdot \frac{1}{\left|V_{i}^{(0)}\right|}+b_{i} \frac{\partial\left|V_{i}\right|^{2}}{\partial V_{r i}} \cdot \frac{1}{\left|V_{i}^{(0)}\right|^{2}}\right]
$$

Em que:

$$
\begin{gathered}
\left|V_{i}\right|^{2}=V_{r i}^{2}+V_{m i}^{2} \rightarrow\left|V_{i}\right|=\sqrt{{V_{r i}}^{2}+V_{m i}{ }^{2}} \\
\left|V_{i}^{(0)}\right|=\sqrt{V_{r i}{ }^{(0)^{2}}+V_{m i}{ }^{(0)^{2}}}
\end{gathered}
$$

Calculando os elementos da equação (4.26)

$$
\begin{aligned}
& \frac{\partial\left|V_{i}\right|}{\partial V_{r i}}=\frac{1}{2}\left[V_{r i}^{2}+V_{m i}^{2}\right]^{-\frac{1}{2}} 2 V_{r i}=\frac{V_{r i}^{(0)}}{\left|V_{i}^{(0)}\right|} \\
& \frac{\partial\left|V_{i}\right|^{2}}{\partial V_{r i}}=2 V_{r i}^{(0)}
\end{aligned}
$$

Reorganizando a equação (4.26):

$$
\frac{\partial P_{c i}}{\partial V_{r i}}=\frac{P_{c i}^{(0)} V_{r i}^{(0)}}{\left|V_{i}^{(0)}\right|^{2}}\left[a_{i}+2 b_{i}\right]=K_{c i 1}
$$


Adotando o mesmo procedimento para os outros termos da matriz, temos:

$$
\begin{gathered}
\frac{\partial P_{c i}}{\partial V_{m i}}=\frac{P_{c i}^{(0)} V_{m i}^{(0)}}{\left|V_{i}^{(0)}\right|^{2}}\left[a_{i}+2 b_{i}\right]=K_{c i 2} \\
\frac{\partial Q_{c i}}{\partial V_{r i}}=\frac{Q_{c i}^{(0)} V_{r i}^{(0)}}{\left|V_{i}^{(0)}\right|^{2}}\left[c_{i}+2 d_{i}\right]=K_{c i 3} \\
\frac{\partial Q_{c i}}{\partial V_{m i}}=\frac{Q_{c i}^{(0)} V_{m i}^{(0)}}{\left|V_{i}^{(0)}\right|^{2}}\left[c_{i}+2 d_{i}\right]=K_{c i 4}
\end{gathered}
$$

A modelagem das cargas pode ser obtida através da variação dos parâmetros $a, b, c$ e $d$ presentes nas equações acima.

Cargas modeladas em potência constante, permitem a simplificação da equação (4.19) para:

$$
\left[\begin{array}{cc}
V_{m i}{ }^{(0)} & V_{r i}{ }^{(0)} \\
-V_{r i}{ }^{(0)} & V_{m i}{ }^{(0)}
\end{array}\right]\left[\begin{array}{c}
\Delta I_{m i} \\
\Delta I_{r i}
\end{array}\right]=-\left[\begin{array}{cc}
I_{r i}{ }^{(0)} & I_{m i}{ }^{(0)} \\
-I_{m i}{ }^{(0)} & I_{r i}{ }^{(0)}
\end{array}\right]\left[\begin{array}{c}
\Delta V_{r i} \\
\Delta V_{m i}
\end{array}\right]
$$

Neste sentido, $a=b=c=d=0$

Seguindo a mesma linha de pensamento, podemos determinar as demais modelagens:

1. Corrente constante, $a=c=1$ e $b=d=0$;

2. Impedância constante, $a=c=0$ e $b=d=1$.

Opções com combinações desses tipos individuais de carga também são possíveis. 


\section{Capítulo 5 REPRESENTAÇÕES, ANÁLISES E RESULTADOS}

\subsection{Introdução}

Nesse capítulo são realizados estudos do problema de oscilações eletromecânicas de baixa frequência explorando o potencial do modelo da matriz jacobiana na forma de sistema descritor apresentada no Capítulo 3. A análise da estabilidade angular a pequenas perturbações é feita a partir da análise modal, e técnicas de resposta no domínio da frequência e do tempo. Toda análise é realizada utilizando o software Matlab. Será utilizado o sistema teste composto por nove barras ('case9') da referência [2] e ilustrado na Figura 5.1. Os dados da linha de transmissão, do gerador, do regulador de tensão e do controlador PSS são apresentados nas Tabelas 5.1 a 5.5.

Tabela 5.1: Dados de ligacão

\begin{tabular}{ccccccc}
\hline de & para & tipo & resistência & reatância & & \\
\hline & & $\begin{array}{r}1=\mathrm{LT} \\
2=\mathrm{TR}\end{array}$ & $\begin{array}{c}(\mathrm{ohm} / \mathrm{km}) \\
(\mathrm{pu})\end{array}$ & $\begin{array}{c}(\mathrm{ohm} / \mathrm{km}) \\
(\mathrm{pu})\end{array}$ & $\begin{array}{c}\text { cap }(\mathrm{nF} / \mathrm{km}) \\
\text { Sn(MVA })\end{array}$ & comp. (km) \\
\hline 7 & 8 & 1 & 0.0749 & 0.6348 & 6.220 & 60.0 \\
8 & 9 & 1 & 0.0630 & 0.5332 & 5.240 & 100.0 \\
7 & 5 & 1 & 0.0677 & 0.3407 & 3.068 & 250.0 \\
5 & 4 & 1 & 0.0529 & 0.4496 & 4.412 & 100.0 \\
4 & 6 & 1 & 0.0600 & 0.3200 & 2.640 & 150.0 \\
6 & 9 & 1 & 0.0688 & 0.2998 & 2.640 & 300.0 \\
1 & 4 & 2 & 0.0000 & 0.0576 & 100 & 0.0 \\
2 & 7 & 2 & 0.0000 & 0.0625 & 100 & 0.0 \\
3 & 9 & 2 & 0.0000 & 0.0586 & 100 & 0.0 \\
\hline
\end{tabular}




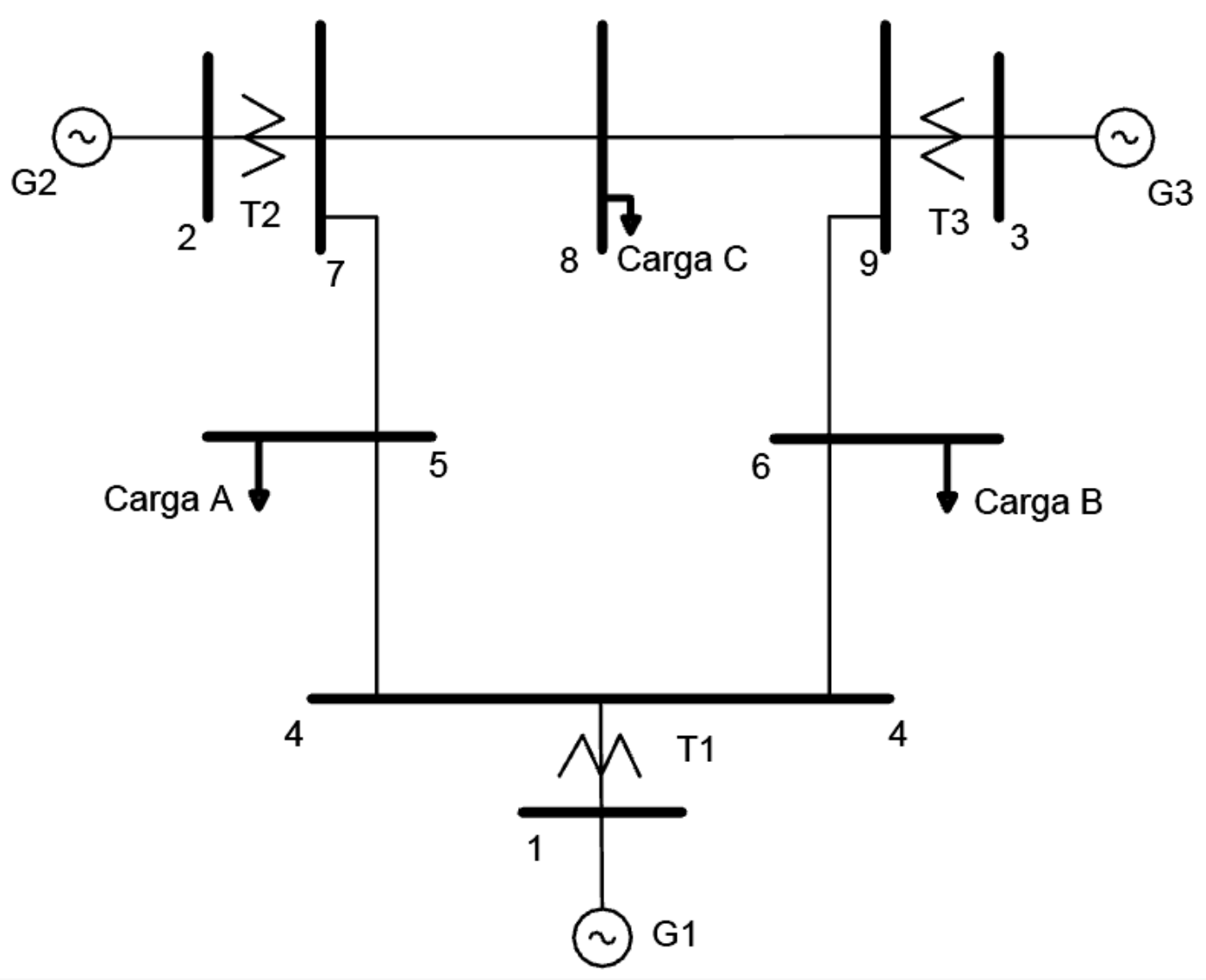

Figura 5.1: Sistema de nove barras da referência [2]

Tabela 5.2: Dados de barra

\begin{tabular}{clrrrrr}
\hline Número & Nome & Tipo & Tensão & P.At.ger & P.At.car & P.Reat.car \\
\hline 1 & & & $(\mathbf{k V})$ & $(\mathbf{p u})$ & $(\mathbf{p u})$ & $(\mathbf{p u})$ \\
\hline 2 & gera1 & 3 & 16.500 & 0.000 & 0.000 & 0.000 \\
3 & gera2 & 2 & 18.000 & 1.630 & 0.000 & 0.000 \\
4 & glfa & 1 & 230.000 & 0.000 & 0.000 & 0.000 \\
5 & beta & 1 & 230.000 & 0.000 & -1.250 & -0.500 \\
6 & gama & 1 & 230.000 & 0.000 & -0.900 & -0.300 \\
7 & delta & 1 & 230.000 & 0.000 & 0.000 & 0.000 \\
8 & eta & 1 & 230.000 & 0.000 & -1.000 & -0.350 \\
9 & lambda & 1 & 230.000 & 0.000 & 0.000 & 0.000 \\
& & & & & & \\
\hline
\end{tabular}


Tabela 5.3: Resultados do Fluxo de carga

\begin{tabular}{|c|c|c|c|c|c|c|c|c|}
\hline \multirow[t]{3}{*}{ Número } & \multirow[t]{3}{*}{ Nome } & \multirow[t]{3}{*}{ Tipo } & \multirow{2}{*}{\multicolumn{2}{|c|}{$\begin{array}{c}\text { Tensão } \\
\text { (Módulo) (Ângulo) }\end{array}$}} & \multicolumn{2}{|c|}{ Geração } & \multicolumn{2}{|c|}{ Carga } \\
\hline & & & & & (Ativo) & (Reativo) & (Ativo) & (Reativo) \\
\hline & & & (pu) & (graus) & $(\mathrm{pu})$ & (pu) & (pu) & (pu) \\
\hline 1 & gera1 & 3 & 1.040 & 0.00 & 0.716 & 0.271 & 0.000 & 0.000 \\
\hline 2 & gera2 & 2 & 1.025 & 9.29 & 1.630 & 0.067 & 0.000 & 0.000 \\
\hline 3 & gera3 & 2 & 1.025 & 4.68 & 0.850 & -0.109 & 0.000 & 0.000 \\
\hline 4 & alfa & 1 & 1.026 & -2.22 & 0.000 & 0.000 & 0.000 & 0.000 \\
\hline 5 & beta & 1 & 0.996 & -3.98 & 0.000 & 0.000 & 1.250 & 0.500 \\
\hline 6 & gama & 1 & 1.013 & -3.67 & 0.000 & 0.000 & 0.900 & 0.300 \\
\hline 7 & delta & 1 & 1.026 & 3.73 & 0.000 & 0.000 & 0.000 & 0.000 \\
\hline 8 & eta & 1 & 1.016 & 0.74 & 0.000 & 0.000 & 1.000 & 0.350 \\
\hline 9 & lambda & 1 & 1.032 & 1.98 & 0.000 & 0.000 & 0.000 & 0.000 \\
\hline
\end{tabular}

Tabela 5.4: Dados dos geradores e controladores do sistema em estudo - Parte 1

\begin{tabular}{cccccccccc}
\hline Gerador & $R a$ & $X q$ & $X^{\prime} d$ & $X^{\prime \prime} d$ & $X^{\prime \prime} q$ & $X l$ & $T^{\prime} d 0$ & $T^{\prime \prime} d 0$ & $X d$ \\
\hline Número & $(\mathbf{p u})$ & $(\mathbf{p u})$ & $(\mathbf{p u})$ & $(\mathbf{p u})$ & $(\mathbf{p u})$ & $(\mathbf{p u})$ & $(\mathbf{p u})$ & $(\mathbf{p u})$ & $(\mathbf{p u})$ \\
\hline 1 & 0 & 0.9690 & 0.0608 & 0.02 & 0.02 & 0.2 & 8.96 & 0.048 & 0.1460 \\
2 & 0 & 0.8645 & 0.1198 & 0.02 & 0.02 & 0.2 & 6.00 & 0.048 & 0.8958 \\
3 & 0 & 1.2578 & 0.1813 & 0.02 & 0.02 & 0.2 & 5.89 & 0.048 & 1.3125 \\
\hline
\end{tabular}

Tabela 5.5: Dados dos geradores e controladores do sistema em estudo - Parte 2

\begin{tabular}{cccccccccc}
\hline Gerador & $T " q 0$ & $D t$ & $H m 1$ & $K w$ & $K a$ & $T a$ & $K p s s$ & $T 1$ & $T 2$ \\
\hline Número & $(\mathbf{p u})$ & $(\mathbf{p u})$ & $(\mathbf{p u})$ & $(\mathbf{p u})$ & $(\mathbf{p u})$ & $(\mathbf{p u})$ & $(\mathbf{p u})$ & $(\mathbf{p u})$ & $(\mathbf{p u})$ \\
\hline 1 & 0.066 & 0 & 23.64 & 5 & 100 & 0.05 & 5 & 0.08 & 0.05 \\
2 & 0.066 & 0 & 6.40 & 5 & 100 & 0.05 & 5 & 0.08 & 0.05 \\
3 & 0.066 & 0 & 3.01 & 5 & 100 & 0.05 & 5 & 0.08 & 0.05 \\
\hline
\end{tabular}




\subsection{Formação da Matriz Jacobiana}

A montagem da matriz de estados $A$ consiste basicamente na inserção sequencial das equações diferenciais que descrevem o comportamento físico da máquina, assim como foi mostrado na Seção 3.2.2, e organizá-las em um vetor de estados, por gerador. Procedimento semelhante pode ser efetuado para os dispositivos componentes do sistema de excitação das máquinas síncronas. Ao final, tem-se um vetor de estado aumentado contendo os estados de todos equipamentos com representação dinâmica. A mesma metodologia é aplicada às variáveis algébricas. De maneira geral, entretanto, estas variáveis são relacionadas com as interconexões da rede aos equipamentos [8]. A Figura 5.2 apresenta um esquema espacial da matriz Jacobiana para a representação do SEP no caso de nove barras em estudo. A ordem sequencial das variáveis de estado e algébricas é apresentada na Seção 3.2.2.

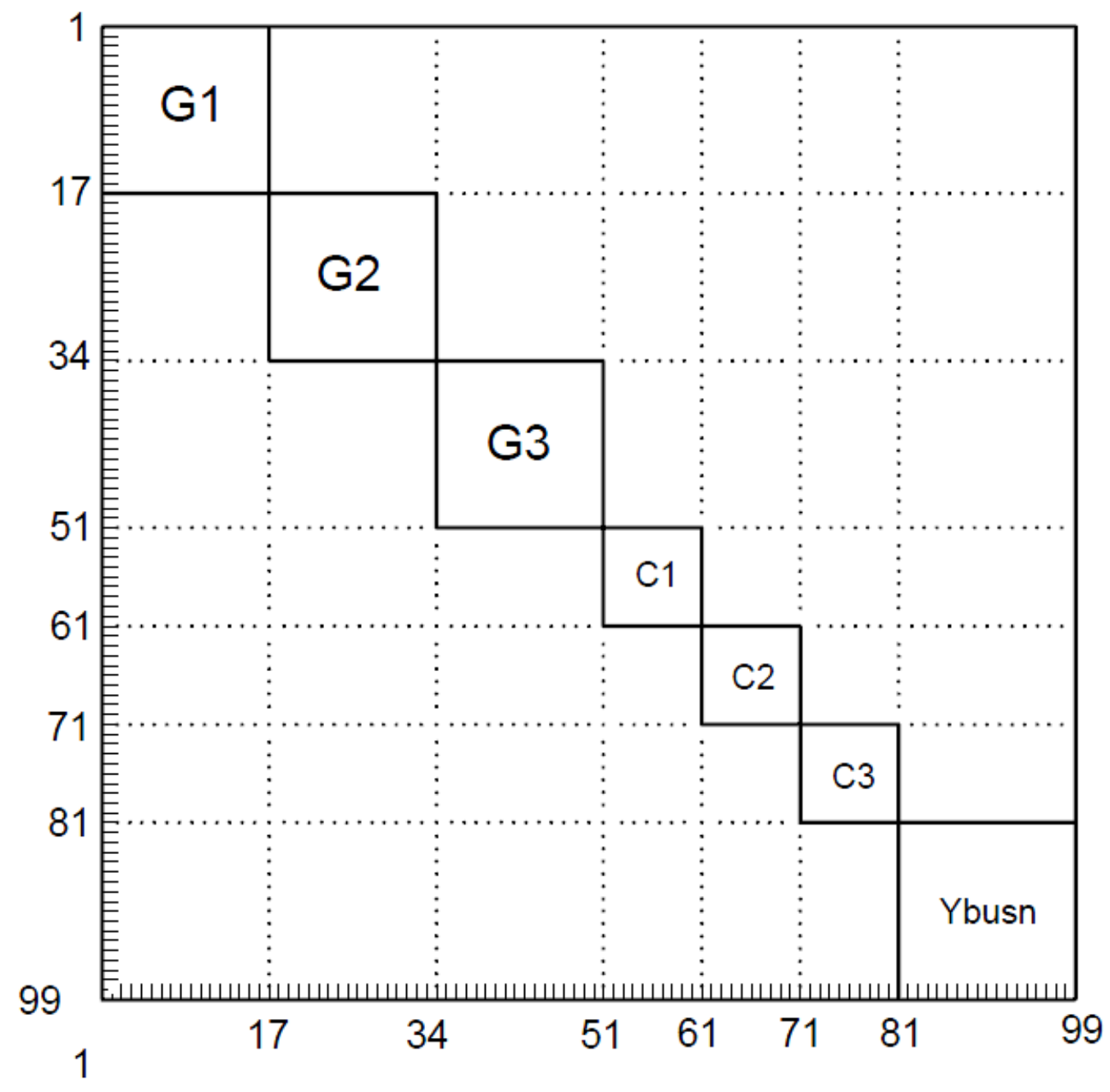

Figura 5.2: Arranjo físico da matriz Jacobiana para o case9 
As partes em destaque representam os locais de maior concentração de elementos nãonulos. Os símbolos $G 1, G 2$ e $G 3$ representam os elementos referentes aos geradores 1, 2 e 3, respectivamente. $C 1, C 2$ e $C 3$ são referentes aos elementos pertencentes ao sistema de excitação dos geradores 1, 2 e 3, respectivamente. Já $Y_{B U S n}$ representa a matriz $Y_{B U S}$ decomposta assim como fora mostrado na equação (3.52) já inclusas as influências das cargas adicionadas aos seus membros. O Apêndice A apresenta um esquemático descritivo da matriz Jacobiana em estudo referente aos elementos do gerador 1 e seu controlador. A matriz $Y_{B U S n}$ também é mostrada assim como a influência das cargas estão determinadas em seus respectivos elementos.

\subsection{Obtenção do Sistema Descritor}

Foi desenvolvido um código que permite obtermos dados do sistema por meio de entrada de dados visual à disposição do usuário. Os passos necessários para a obtenção do sistema descritor do SEP a partir do software desenvolvido são apresentados na sequência.

1. Inicializar o software MATLAB;

2. Definir como workspace a pasta 'Metodo';

3. Executar a função startup;

4. Executar a função 'entrada'. Neste ponto, a interface gráfica de entrada de dados irá ser executada. A Figura 5.3 mostra esta janela.

5. Realizar a inserção de dados na tabela;

6. Clicar no botão Salvar Dados;

7. Definir as constantes referentes à modelagem das cargas assim como fora definido na Seção 4.3;

8. Clicar no botão executar; 


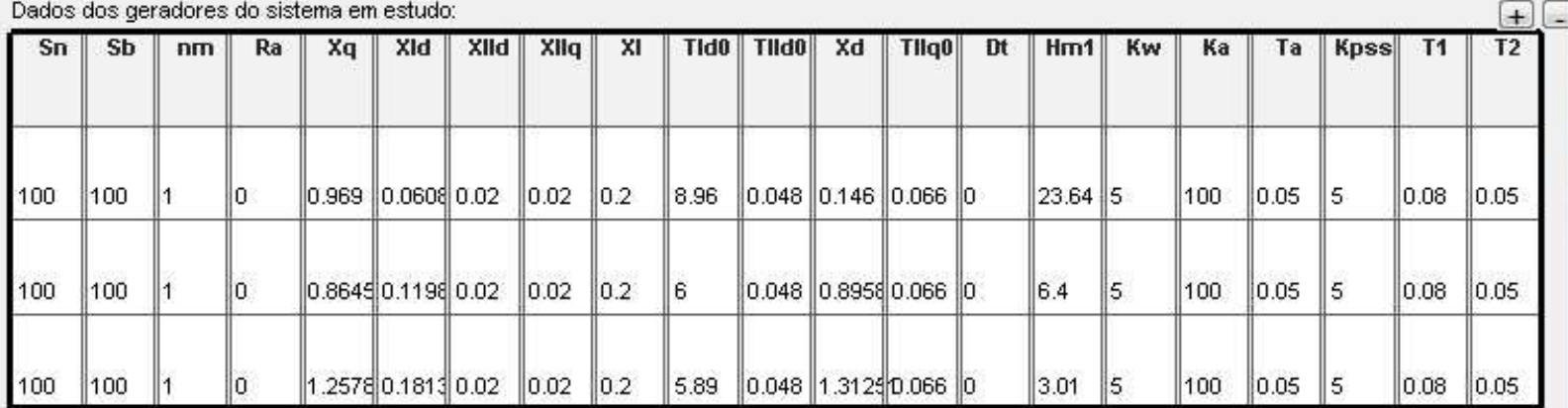

Salvar Dados

Escolha o valor das constantes que serẫo utilizadas no modelamento das cargas:

Constante a:

Constante $b$ :

Constante c:

Constante d:

Executar

Figura 5.3: Interface gráfica de entrada de dados

\subsection{Análise Modal, da Resposta no Tempo e em Frequência}

Modelado o Sistema Elétrico de Potência, podemos realizar a análise dinâmica. Neste sentido, realizamos, com o auxílio do MATLAB, o cálculo dos autovalores da matriz Jacobiana $A$ obtida pelo nosso software e comparamos com os resultados obtidos por uma simulação correspondente no PACDYN. Este procedimento foi desenvolvido apenas para o sistema particular descrito como 'case9' do programa MATPOWER [2].Os resultados estão disponíveis na Tabela 5.6.

Tabela 5.6: Resultados obtidos para o case9

\section{Autovalores}

\begin{tabular}{ccc}
\hline Projeto & PacDyn & Erro \\
\hline$-1.8900 \mathrm{e}+002$ & $-1.8900 \mathrm{e}+002$ & $\mathrm{e}-004$ \\
\hline$-1.2428 \mathrm{e}+002$ & $-1.2428 \mathrm{e}+002$ & $\mathrm{e}-004$ \\
\hline
\end{tabular}


Tabela 5.6 - Resultados obtidos para o case9 - Continuação

Autovalores

\begin{tabular}{|c|c|c|}
\hline Projeto & PacDyn & Erro \\
\hline$-9.2933 \mathrm{e}+001$ & $-9.2933 \mathrm{e}+001$ & $\mathrm{e}-005$ \\
\hline$-2.3635 \mathrm{e}+001+1.8738 \mathrm{e}+001 \mathrm{i}$ & $-2.3635 \mathrm{e}+001+1.8738 \mathrm{e}+001 \mathrm{i}$ & e-004 \\
\hline$-2.3635 \mathrm{e}+001-1.8738 \mathrm{e}+001 \mathrm{i}$ & $-2.3635 \mathrm{e}+001-1.8738 \mathrm{e}+001 \mathrm{i}$ & e-004 \\
\hline$-2.6854 \mathrm{e}+000+1.1001 \mathrm{e}+001 \mathrm{i}$ & $-2.6854 \mathrm{e}+000+1.1001 \mathrm{e}+001 \mathrm{i}$ & $\mathrm{e}-004$ \\
\hline$-2.6854 \mathrm{e}+000-1.1001 \mathrm{e}+001 \mathrm{i}$ & $-2.6854 \mathrm{e}+000-1.1001 \mathrm{e}+001 \mathrm{i}$ & e-004 \\
\hline$-1.1149 \mathrm{e}+001+1.1840 \mathrm{e}+001 \mathrm{i}$ & $-1.1149 \mathrm{e}+001+1.1840 \mathrm{e}+001 \mathrm{i}$ & e-004 \\
\hline$-1.1149 \mathrm{e}+001-1.1840 \mathrm{e}+001 \mathrm{i}$ & $-1.1149 \mathrm{e}+001-1.1840 \mathrm{e}+001 \mathrm{i}$ & e-004 \\
\hline$-2.7966 \mathrm{e}+001$ & $-2.7966 \mathrm{e}+001$ & e-004 \\
\hline$-2.3418 \mathrm{e}+001+5.4801 \mathrm{e}+000 \mathrm{i}$ & $-2.3418 \mathrm{e}+001+5.4803 \mathrm{e}+000 \mathrm{i}$ & e-004 \\
\hline$-2.3418 \mathrm{e}+001-5.4801 \mathrm{e}+000 \mathrm{i}$ & $-2.3418 \mathrm{e}+001-5.4803 \mathrm{e}+000 \mathrm{i}$ & e-004 \\
\hline$-1.9591 \mathrm{e}+000+6.2137 \mathrm{e}+000 \mathrm{i}$ & $-1.9592 \mathrm{e}+000+6.2138 \mathrm{e}+000 \mathrm{i}$ & $\mathrm{e}-004$ \\
\hline$-1.9591 \mathrm{e}+000-6.2137 \mathrm{e}+000 \mathrm{i}$ & $-1.9592 \mathrm{e}+000-6.2138 \mathrm{e}+000 \mathrm{i}$ & e-004 \\
\hline$-1.3643 \mathrm{e}+001$ & $-1.3643 \mathrm{e}+001$ & e-005 \\
\hline$-2.0710 \mathrm{e}+001$ & $-2.0710 \mathrm{e}+001$ & e-005 \\
\hline$-1.9995 \mathrm{e}+001$ & $-1.9995 \mathrm{e}+001$ & e-006 \\
\hline$-4.2991 \mathrm{e}+000$ & $-4.2990 \mathrm{e}+000$ & e-005 \\
\hline$-5.9988 \mathrm{e}-001$ & $-5.9986 \mathrm{e}-001$ & e-005 \\
\hline
\end{tabular}

Uma análise dos resultados obtidos mostra que não houve discrepâncias significativas entre os valores. A simulação indica que o sistema é estável ao passo que não existem autovalores reais positivos. Visando confirmar a estabilidade do sistema, foi realizada simulação no tempo. As Figuras 5.4 e 5.5 mostram os resultados obtidos. 


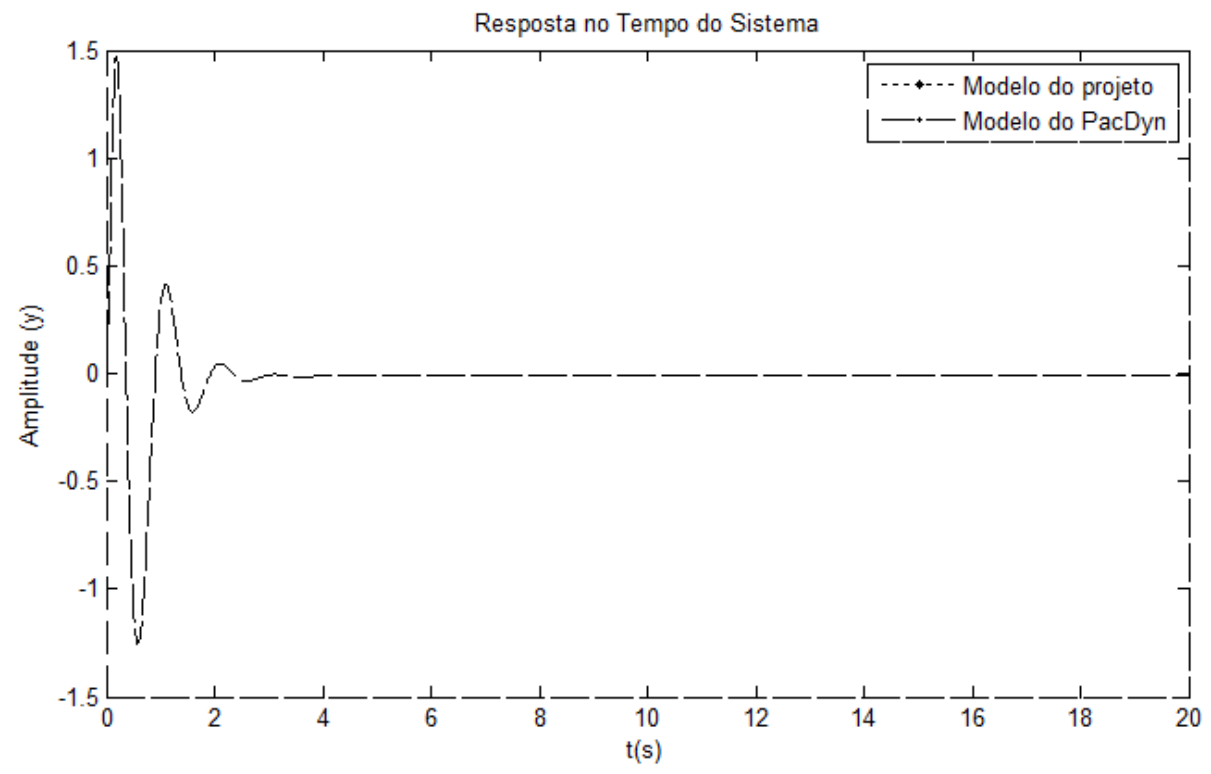

Figura 5.4: Resposta a um degrau para o case9

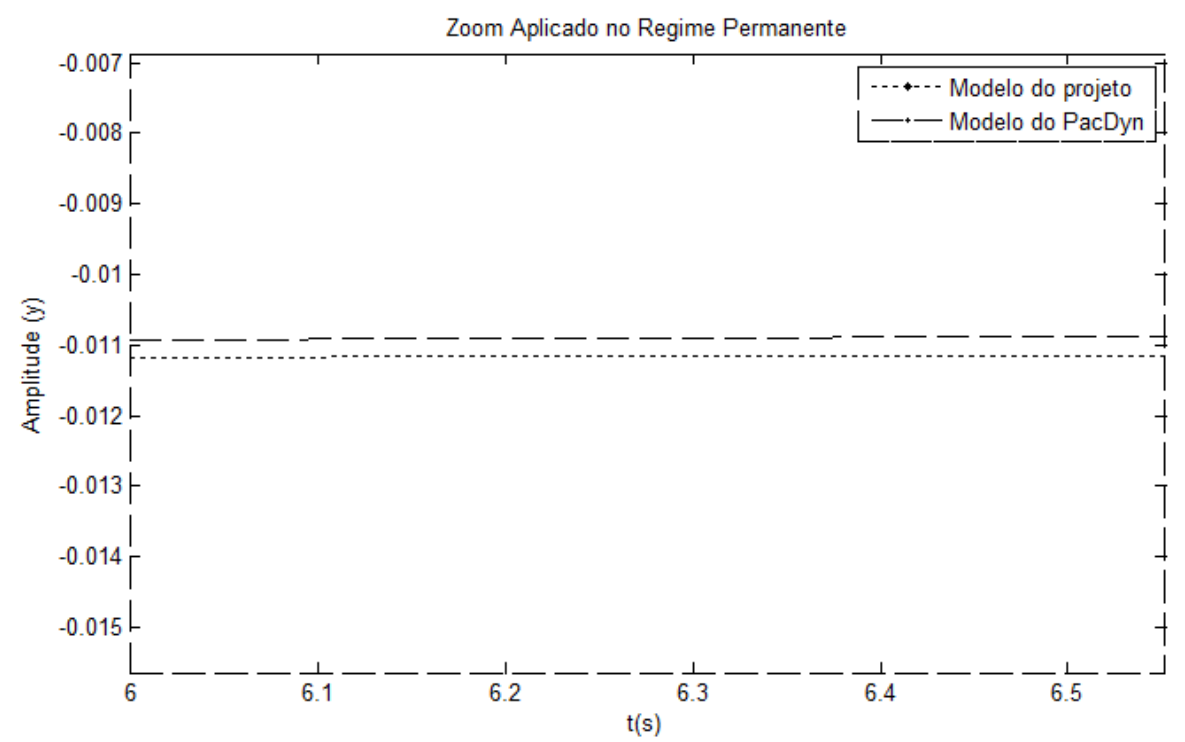

Figura 5.5: Zoom aplicado em regime permanente

Pode-se observar que o sistema é estável haja vista que mesmo após a inserção de uma peperturbação o estado do sistema permanece dentro dos limites especificados, após um certo período de tempo. Percebe-se, por outro lado, que as curvas referentes ao projeto e ao PACDYN se sobrepuseram o que por sua vez indica que o modelo implementado em nosso projeto é compatível com modelos existentes na literatura. 
Partimos agora para a obtenção da resposta em frequência do sistema em estudo, considerando os resultados obtidos no PACDYN e em nosso projeto.

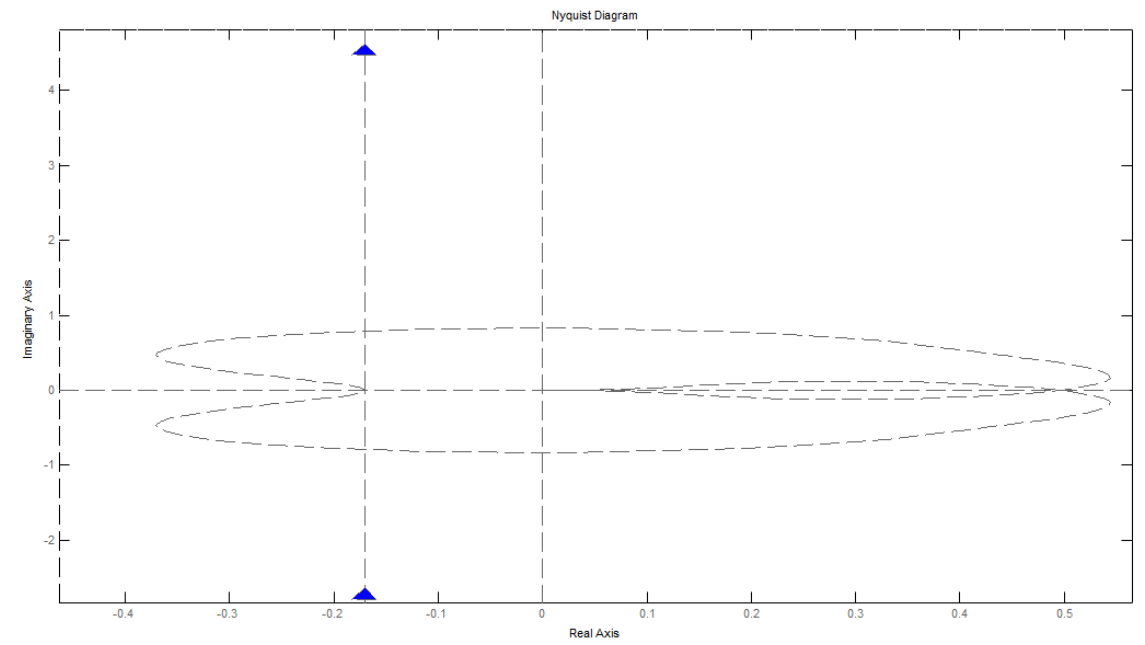

Figura 5.6: Diagrama de Nyquist plotado com a saída do projeto

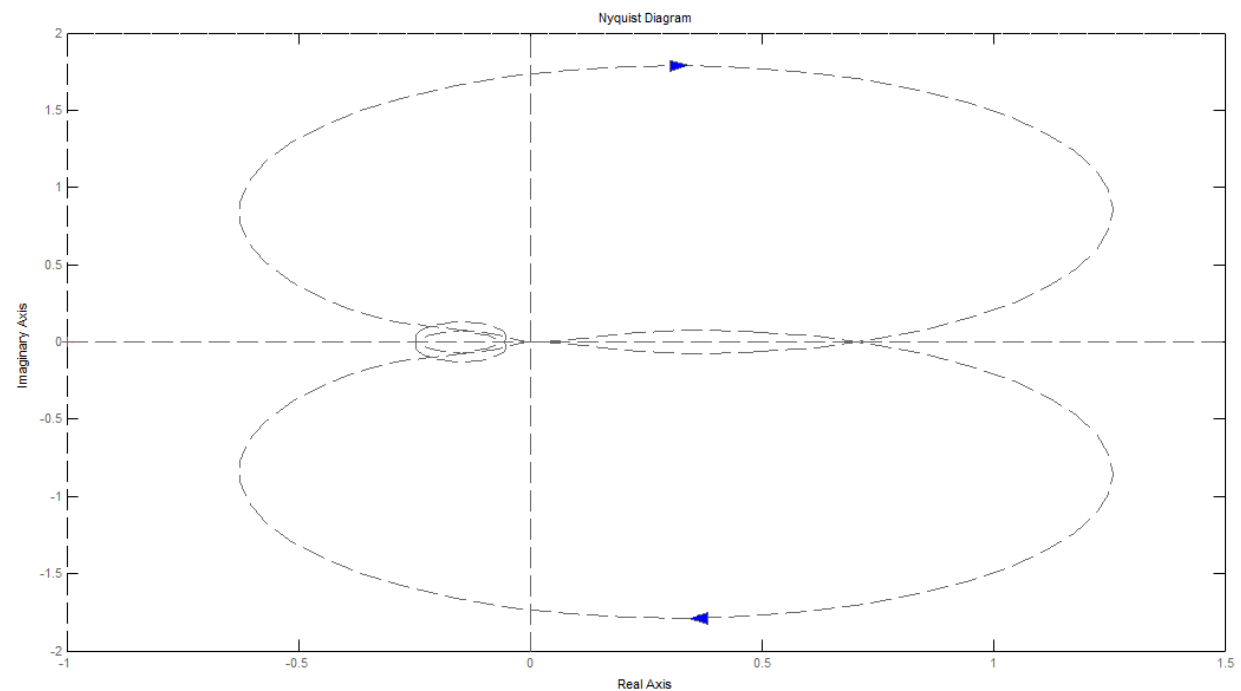

Figura 5.7: Diagrama de Nyquist plotado com a saída do PACDYN

As Figuras 5.6 e 5.6 mostram as respostas em frequência em termos de Diagrama de Nyquist. Observando-as é possível perceber que em ambos os casos não há envolvimento da origem da curva, nem do ponto $(-1+j 0)$. Assim, como não existem pólos (ou autovalores) instáveis e nem zeros no semiplano direito do plano $s$, o sistema é estável. 


\section{Capítulo 6 CONCLUSÕES E SUGESTÕES PARA TRABALHOS FUTUROS}

Foi desenvolvido um sistema computacional para o auxilio na análise de estabilidade a pequenos sinais de sistemas elétricos de potência, e opções de dispositivos controladores de tensão e dispositivos PSS. Já a ação dos reguladores de velocidade das máquinas síncronas não foi considerada no estudo de pequenos sinais neste trabalho. O programa tem por finalidade construir o modelo dinâmico do sistema elétrico em estudo para que assim, com o auxilio do MATLAB, o usuário consiga realizar as análises modais e em frequência. Todos os resultados das rotinas implementadas foram confrontados com os resultados obtidos na literatura e não foram encontrados desvios significativos.

O programa desenvolvido apresenta uma interface gráfica para facilitar as entradas de dados dos geradores e seus controladores. Assim, torna-se fácil a alteração de praticamente todos os parâmetros de entrada o que por sua vez permitirá realizar diversas simulações.

O código apresenta uma estrutura versátil para a representação do SEP no que se refere à escolha do modelo de máquina síncrona que o usuário tiver interesse em analisar. Isto porque todas as variáveis de estado dos principais modelos foram previstas e inseridas na formulação da matriz jacobiana. O usuário necessita apenas realizar a inserção das equações pertinentes a cada modelo que se deseje implementar nas respectivas variáveis de estado previstas no código. Além disso, ele oferece livre acesso ao código fonte, pois foi desenvolvido em MATLAB.

Podemos dizer que a implementação da interface gráfica em ambiente MATLAB não apresentou total interação com o usuário no que se refere a obtenção de tabelas e gráficos utilizados na análise do modelo dinâmico. Alem disso, há apenas um modelo de controlador PSS e de regulador RT previsto no código. 
Pode-se implementar algumas melhorias e procedimentos, a fim de aumentar o campo de atuação do programa, tornando-o mais abrangente e versátil:

$\diamond \mathrm{O}$ código poderá incluir novos dispositivos relacionados a sistemas de energia elétrica como reguladores de velocidade, dispositivos FACTS e novos modelos para os reguladores de tensão e controladores PSS;

$\diamond$ Desenvolver uma interface gráfica amigável para a obtenção e análise de resultados referentes à estabilidade dinâmica de sistemas de potência;

$\diamond$ Em versões futuras o software poderá incluir um módulo de análise de estabilidade transitória. 


\section{REFERÊNCIAS BIBLIOGRÁFICAS}

[1] P. Kundur. Power System Stability and Control. EPRI Power System Engineering Series. McGraw-Hill, 1994.

[2] P. M. Anderson and A. A. Fouad. Power System Control and Stability IEEE Press, 1994.

[3] W. S. Peter and M. A. Pai. Power Systems Dynamics and Stability Prentice-Hall, New Jersey, 1998.

[4] M. K. Pal. Power System Stability, Lecture Notes, M. K. Pal Consulting, Dayton, New Jersey, USA.

[5] F. P. DeMello and C. Concordia. Concepts of Synchronous Machine Stability as Affected by excitation control. IEEE Trans. on PAS, v. 88, n. 4, pp. 316-329, Apr. 1969.

[6] CEPEL. Manual do usuário do PacDyn. Centro de Pesquisas de Energia Elétrica, Rio de Janeiro, Brasil, 2002.

[7] K. Ogata. Modern Control Engineering. Prentice Hall International, 1970.

[8] F. D. Freitas. Ajuste de Estabilizadores de Sistemas de Potência Via Controle Ótimo com Restrições Estruturais. Tese de Doutorado, UFSC, 1995.

[9] H. E. Peña. Controle Ótimo Descentralizado Aplicado ao Projeto e Coordenação de Estabilizadores de Sistemas de Potência. Tese de Doutorado, UFSC, 1992.

[10] N. Martins. Efficient Eigenvalue and Frequency Response Methods Applied to Power System Small-signal Stability Studies. IEEE Trans. on Power Systems, 1(1):217-226, February 1986.

[11] IEEE Committee report. Computer representation of excitation systems. IEEE Transactions on Power Apparatus and Systems, PAS-87(6):1460-1464, June 1968. 


\section{Apêndice A ESQUEMÁTICO MATRIZ A PARA O CASE 9}

Neste Apêndice apresentam-se os elementos da matriz Jacobiana $A$ referentes apenas ao gerador 1. Os demais, poderão ser obtidos de modo similar.

MATRIZ A

Linha 1 - Correspondente a $E_{q}^{\prime \prime}$ :

$A(1,1)=\left[\frac{\left(X_{d}^{\prime \prime}-X_{l}\right)\left(X_{d}-X_{d}^{\prime}\right)}{T_{d 0}^{\prime}\left(X_{d}^{\prime}-X_{l}\right)}-\frac{1}{T_{d 0}^{\prime \prime}}\right]$

$A(1,3)=\left[\frac{-\left(X_{d}^{\prime \prime}-X_{l}\right)\left(X_{d}-X_{l}\right)}{T_{d 0}^{\prime}\left(X_{d}^{\prime}-X_{l}\right)^{2}}\right]$

$A(1,7)=\left[\frac{-\left(X_{d}^{\prime \prime}-X_{l}\right)\left(X_{d}-X_{d}^{\prime}\right)\left(X_{d}^{\prime \prime}-X_{l}\right)}{T_{d 0}^{\prime}\left(X_{d}^{\prime}-X_{l}\right)^{2}}-\frac{\left(X_{d}^{\prime}-X_{d}^{\prime \prime}\right)}{T_{d 0}^{\prime \prime}}\right]$

$A(1,9)=-\left[\frac{\left(X_{d}^{\prime \prime}-X_{l}\right)}{T_{d 0}^{\prime}\left(X_{d}^{\prime}-X_{l}\right)}\right]$

$A(1,11)=\left[\frac{\left(X_{d}^{\prime \prime}-X_{l}\right)}{T_{d 0}^{\prime}\left(X_{d}^{\prime}-X_{l}\right)}\right]$

Linha 2 - Correspondente a $E_{d}^{\prime \prime}$ :

$A(2,2)=\left[\frac{-1}{T_{q 0}^{\prime \prime}}\right]$
$A(2,8)=\left[\frac{\left(X_{l}-X_{d}^{\prime \prime}\right)}{T_{q 0}^{\prime \prime}}\right]$

Linha 3 - Correspondente a $E_{q}^{\prime}$ :

$$
\begin{aligned}
& A(3,1)=\left[\frac{\left(X_{d}-X_{d}^{\prime}\right)}{T_{d 0}^{\prime}\left(X_{d}^{\prime}-X_{l}\right)}\right] \\
& A(3,3)=\left[\frac{\left(X_{l}-X_{d}\right)}{T_{d 0}^{\prime}\left(X_{d}^{\prime}-X_{l}\right)}\right]
\end{aligned}
$$




$$
\begin{aligned}
& A(3,7)=\left[-\frac{\left(X_{d}-X_{d}^{\prime}\right)\left(X_{s}^{\prime \prime}-X^{\prime}\right)}{T_{d 0}^{\prime}\left(X_{d}^{\prime}-X^{\prime}\right)}\right] \\
& A(3,9)=\frac{-1}{T_{d 0}^{\prime}} \\
& A(3,11)=\frac{1}{T_{d 0}^{\prime}}
\end{aligned}
$$

Linha 4 - Correspondente a $E_{d}^{\prime}$ :

$A(4,4)=-1$

Pode-se pereceber que esta linha nos indica $0=-E_{d}^{\prime}$. Em princípio isto pode nos parecer estranho, mas este será um artifício utilizado em nosso software para aquelas linhas da matriz de estados que representem equações algébricas ou diferenciais que não estiverem presentes no modelo em estudo. Isto permite que outros modelos de máquinas síncronas sejam estudados e implementados haja vista que o espaço de todas as possíveis variáveis em nossa matriz de estados A são previamente inseridos. Isto não trará maiores prejuízos ao algoritmo que está sendo implementado.

Linha 5 -Correspondente a $\omega$ :

$$
\begin{aligned}
& A(5,5)=\frac{-D_{t}}{2 H} \\
& A(5,6)=\frac{-1}{2 H}\left[\cos \delta_{0}\left(I_{d 0} V_{r i}^{(0)}+I_{q 0} V_{m i}^{(0)}\right)+\sin \delta_{0}\left(I_{d 0} V_{m i}^{(0)}-I_{q 0} V_{r i}^{(0)}\right)\right] \\
& A(5,12)=\frac{1}{2 H} \\
& A(5,62)=\frac{-1}{2 H}\left(I_{d 0} \sin \delta_{0}+I_{q 0} \cos \delta_{0}\right) \\
& A(5,63)=\frac{1}{2 H}\left(I_{d 0} \cos \delta_{0}-I_{q 0} \sin \delta_{0}\right)
\end{aligned}
$$

Linha 6 - Correspondente a $\delta$ :

$A(6,5)=\omega_{o}$

Linha 7 - Correspondente a $I_{d}$ :

$$
\begin{aligned}
& A(7,1)=-1 \\
& A(7,6)=\left(V_{m i}^{(0)} \cos \delta_{0}-V_{r i}^{(0)} \sin \delta_{0}\right)
\end{aligned}
$$


$A(7,7)=X_{d}^{\prime \prime}$

$A(7,8)=R_{a}$

$A(7,16)=\sin \delta_{0}$

$A(7,17)=-\cos \delta_{0}$

Linha 8 -Correspondente a $I_{q}$ :

$A(8,2)=-1$

$A(8,6)=\left(V_{m i}^{(0)} \sin \delta_{0}+V_{r i}^{(0)} \cos \delta_{0}\right)$

$A(8,7)=R_{a}$

$A(8,8)=-X_{q}^{\prime \prime}$

$A(8,62)=\sin \delta_{0}$

$A(8,63)=-\cos \delta_{0}$

Linha 9 -Correspondente a $S_{d}$ :

$A(9,9)=1$

Linha 10 -Correspondente a $S_{q}$ :

$A(10,10)=1$

Linha 11 - Correspondente a $E_{f d}$ :

$A(11,11)=-1$

$A(11,57)=1$

Linha 12-Correspondente a $P_{m}$ :

$A(12,12)=-1$

Linha 13 -Correspondente a $V_{P S S}$ :

$A(13,13)=-1$ 
$A(13,61)=1$

Linha 14 - Correspondente a $V_{R E F}$ :

$A(14,14)=-1$

Linha 15 - Correspondente a $P_{R E F}$ :

$A(15,15)=-1$

Linha $(82+2 i)$ em diante - Correspondente a $V_{r i}$ :

$A(16,6)=-\left(I_{d 0} \sin \delta_{0}+I_{q 0} \cos \delta_{0}\right)$

$A(16,7)=-\cos \delta_{0}$

$A(16,8)=\sin \delta_{0}$

$A(16,16)=-1$

$A(82+2 j, 82+2 i)=\left[-B_{i k}+k_{c i 1}\right]$

$A[82+2 j, 82+(2 i+1)]=\left[-G_{i k}+K_{c i 2}\right]$

Com $i$ e $j$ variando de zero ao número de barras menos um (i,j $=0$ :Nbar-1).

As constantes $K_{c i 1}$ e $K_{c i 2}$ irão introduzir a influência das cargas nos elementos da matriz $Y_{B U S}$ correspondentes à barra i, caso nela exista carga. Tais constantes serão determinadas na próxima seção.

Linha $(83+2 i)$ em diante - Correspondente a $V_{m i}$ :

$A(16,6)=\left(I_{d 0} \cos \delta_{0}-I_{q 0} \sin \delta_{0}\right)$

$A(17,7)=\sin \delta_{0}$

$A(17,8)=\cos \delta_{0}$

$A(17,17)=-1$

$A[82+(2 j+1), 62+2 i]=\left[-G_{i k}+K_{c i 3}\right]$

$A[83+(2 j+1), 62+(2 i+1)]=\left[B_{i k}+K_{c i 4}\right]$ 
Com $i$ e $j$ variando de zero ao número de barras menos um (i $=0$ :Nbar-1).

As constantes $K_{c i 3}$ e $K_{c i 4}$ irão introduzir a influência das cargas nos elesmentos da matriz $Y_{B U S}$ correspondentes à barra i, caso nela exista carga. Tais constantes serão determinadas na próxima seção.

Linha 52 - correspondente a $V_{R E F}^{R T}$ :

$A(52,14)=1$

$A(52,52)=-1$

Linha 53 - Correspondente a $V_{t}^{R T}$ :

$A(53,53)=-1$

$A(53,62)=1$

$A(53,63)=1$

Linha 54 - Correspondente a $V_{P S S}^{R T}$ :

$A(54,13)=1$

$A(54,54)=-1$

Linha 55 - Correspondente a $X_{1}^{R T}$ :

$A(55,52)=1$

$A(55,53)=-1$

$A(55,54)=1$

$A(55,55)=-1$

Linha 56 - Correspondente a $X_{1}^{R T}$ :

$$
\begin{aligned}
& A(56,55)=\frac{k_{a}}{T_{a}} \\
& A(56,56)=\frac{-1}{T_{a}}
\end{aligned}
$$


Linha 57-Correspondente a $E_{f d}^{R T}$ :

$A(57,56)=1$

$A(57,57)=-1$

Linha 58 - Correspondente a D $\omega^{P S S}$ :

$A(58,5)=1$

$A(58,58)=-1$

Linha 59 - Correspondente a $X_{0006}^{P S S}$.

$A(59,58)=\left[\frac{\left(T_{2}-T_{1}\right)}{T_{2}^{2}}\right]$

$A(59,59)=\frac{-1}{T_{2}}$

Linha 60 - Correspondente a $X_{1}^{P S S}$ :

$A(60,58)=\frac{T_{1}}{T_{2}}$

$A(60,59)=1$

$A(60,60)=-1$

Linha 61 - Correspondente a $V_{P S S}^{P S S}$ :

$A(61,60)=K_{P S S}$

$A(61,60)=-1$ 


\section{Apêndice B CÓDIGO MATLAB IMPLEMENTADO}

Abaixo, listamos o código fonte do programa principal implementado em nosso projeto:

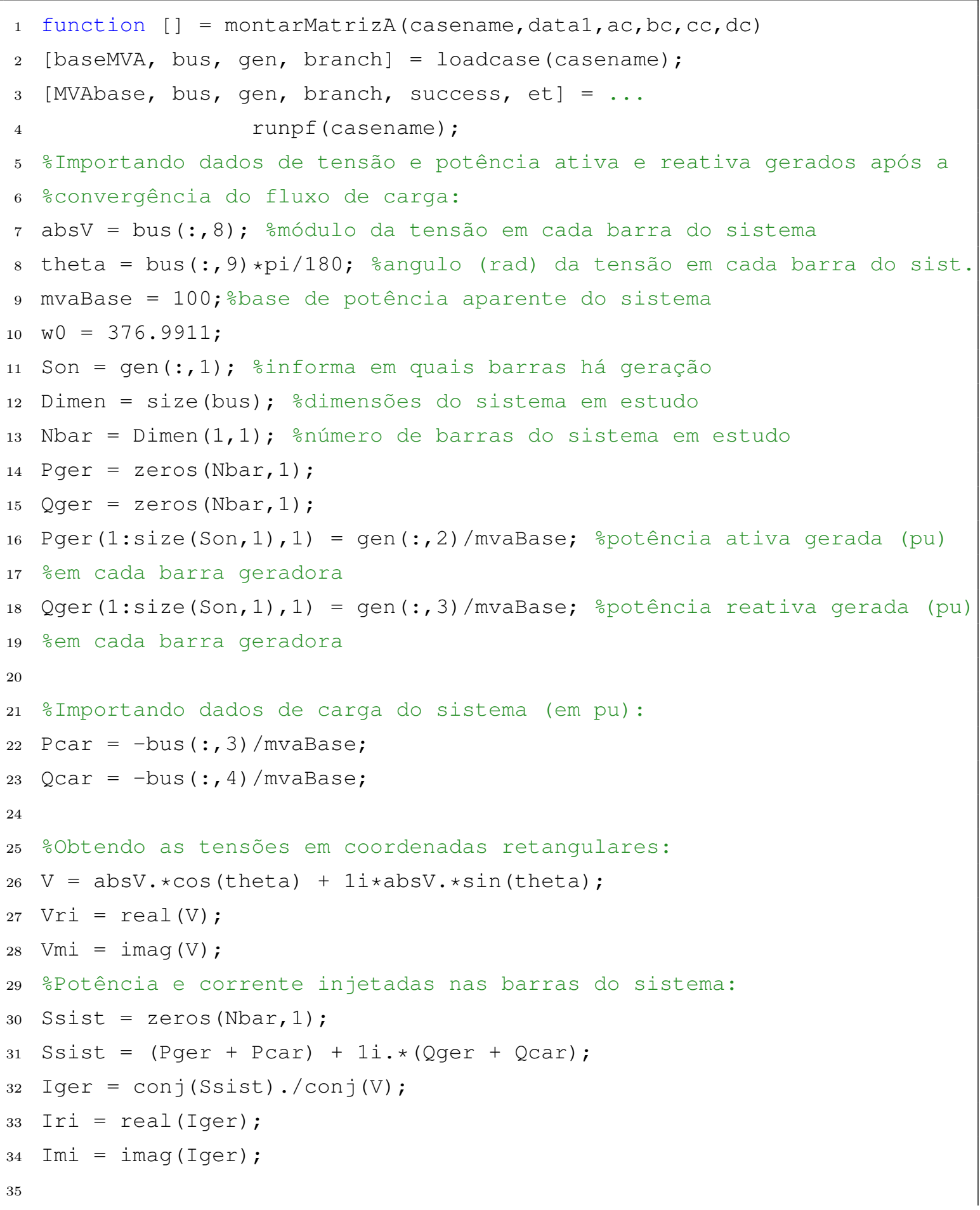




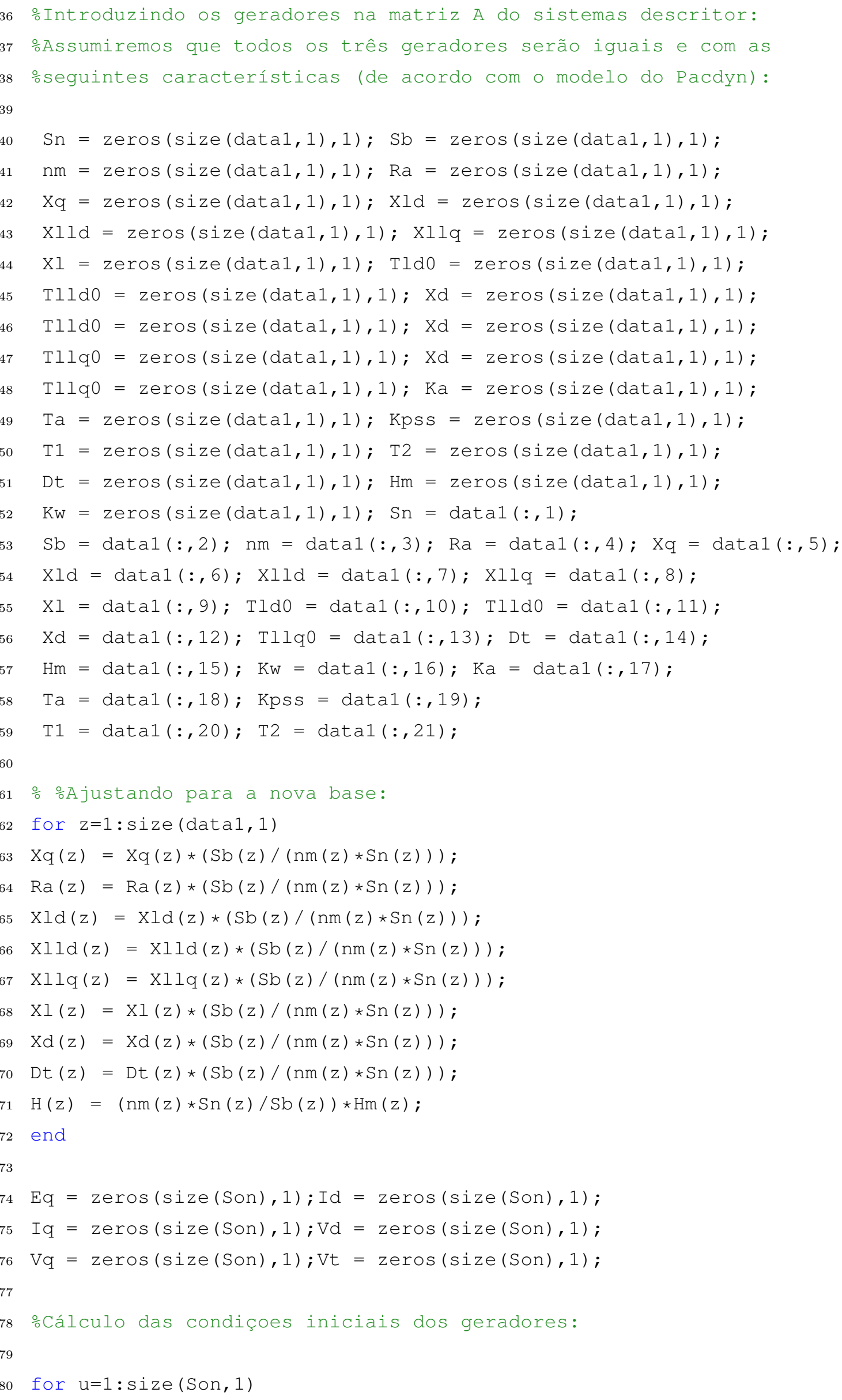




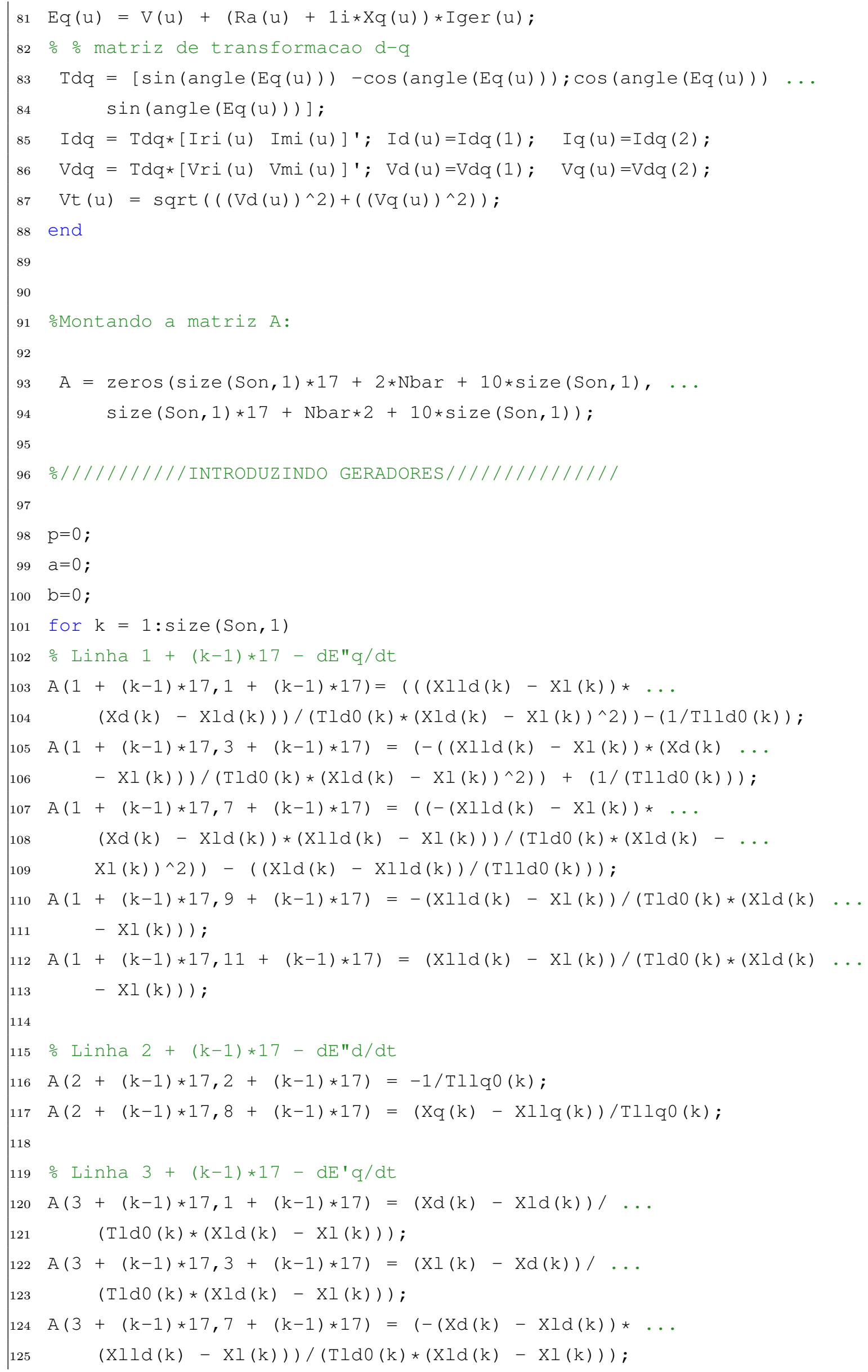




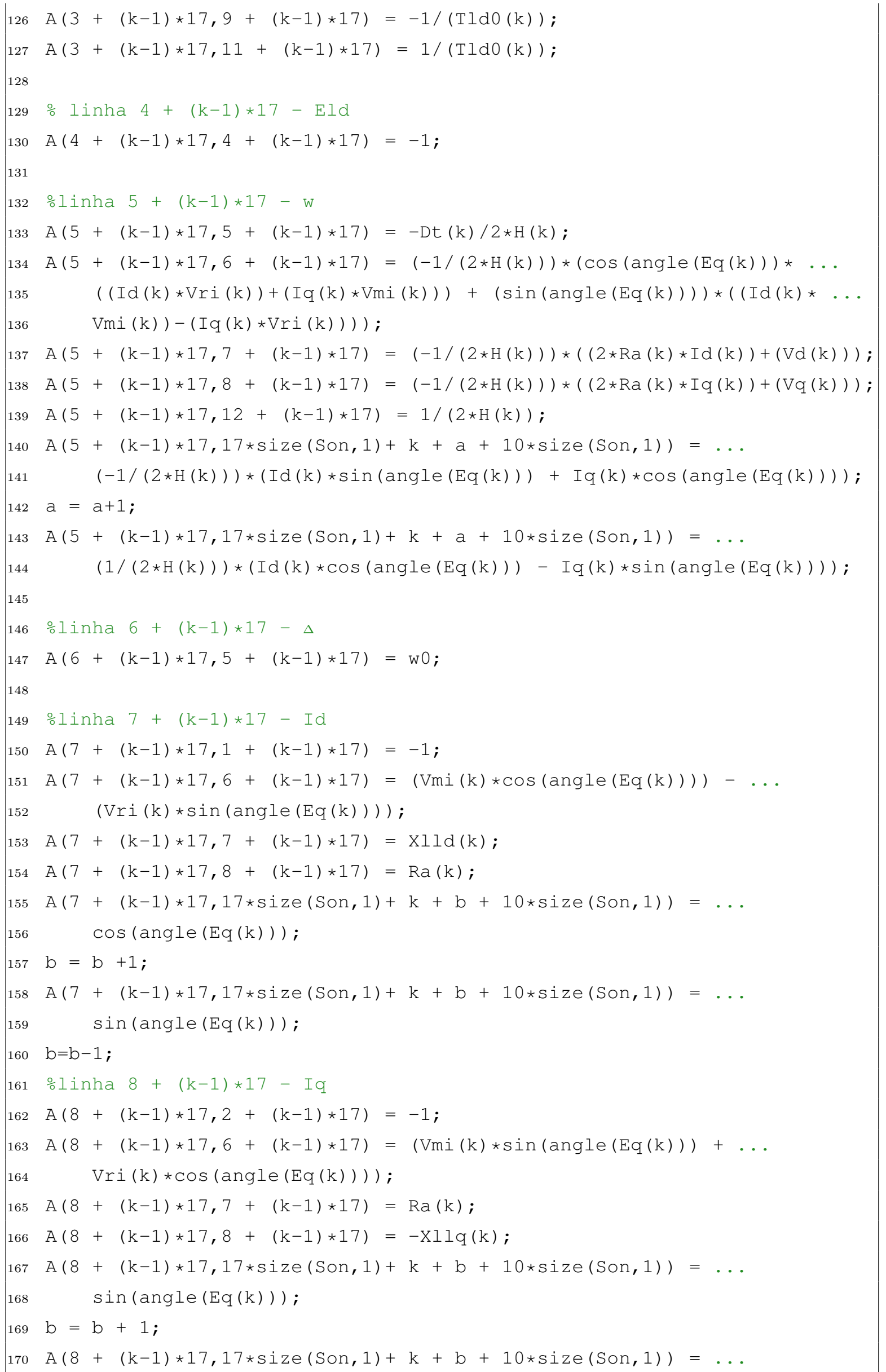




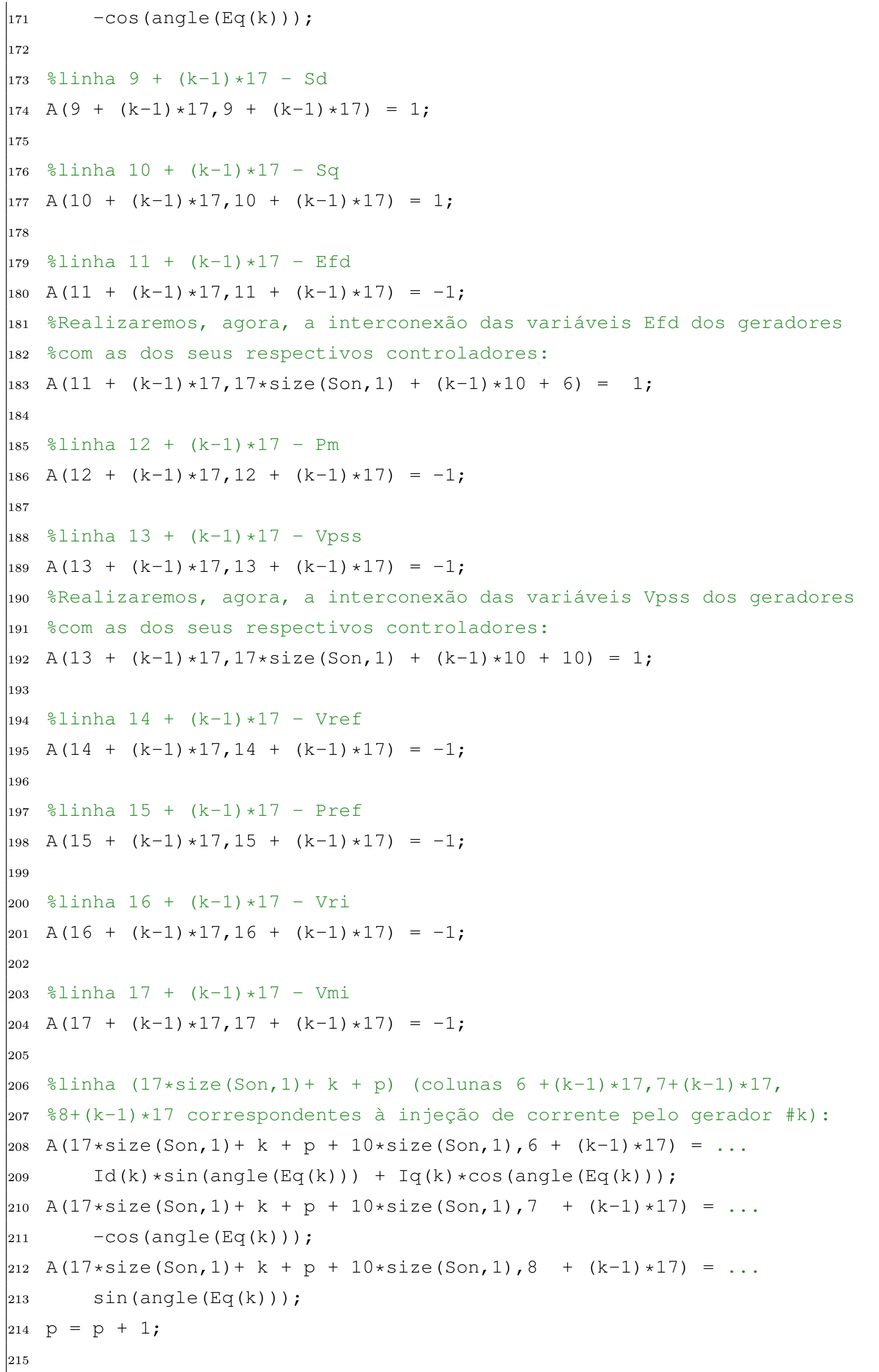




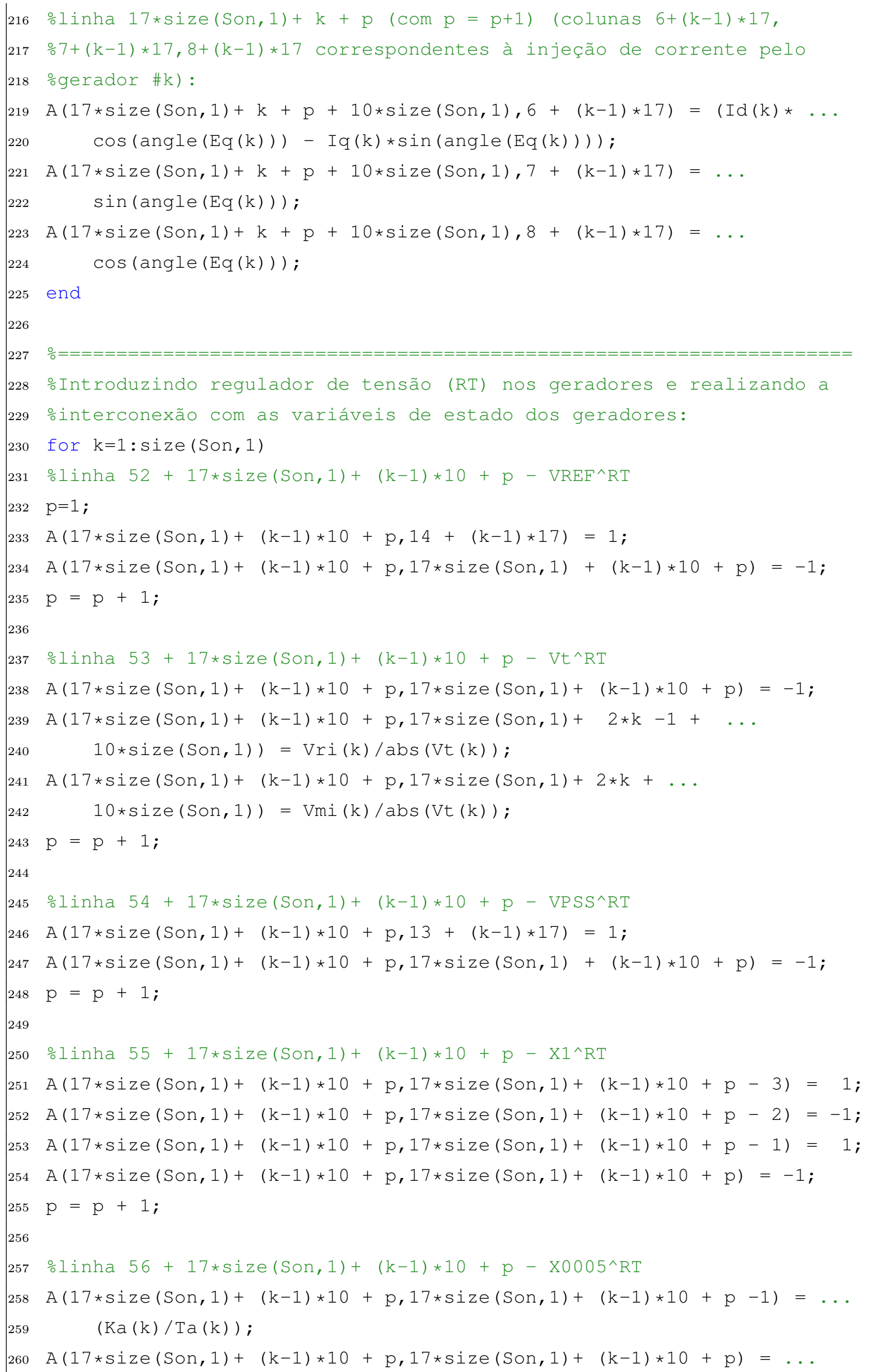




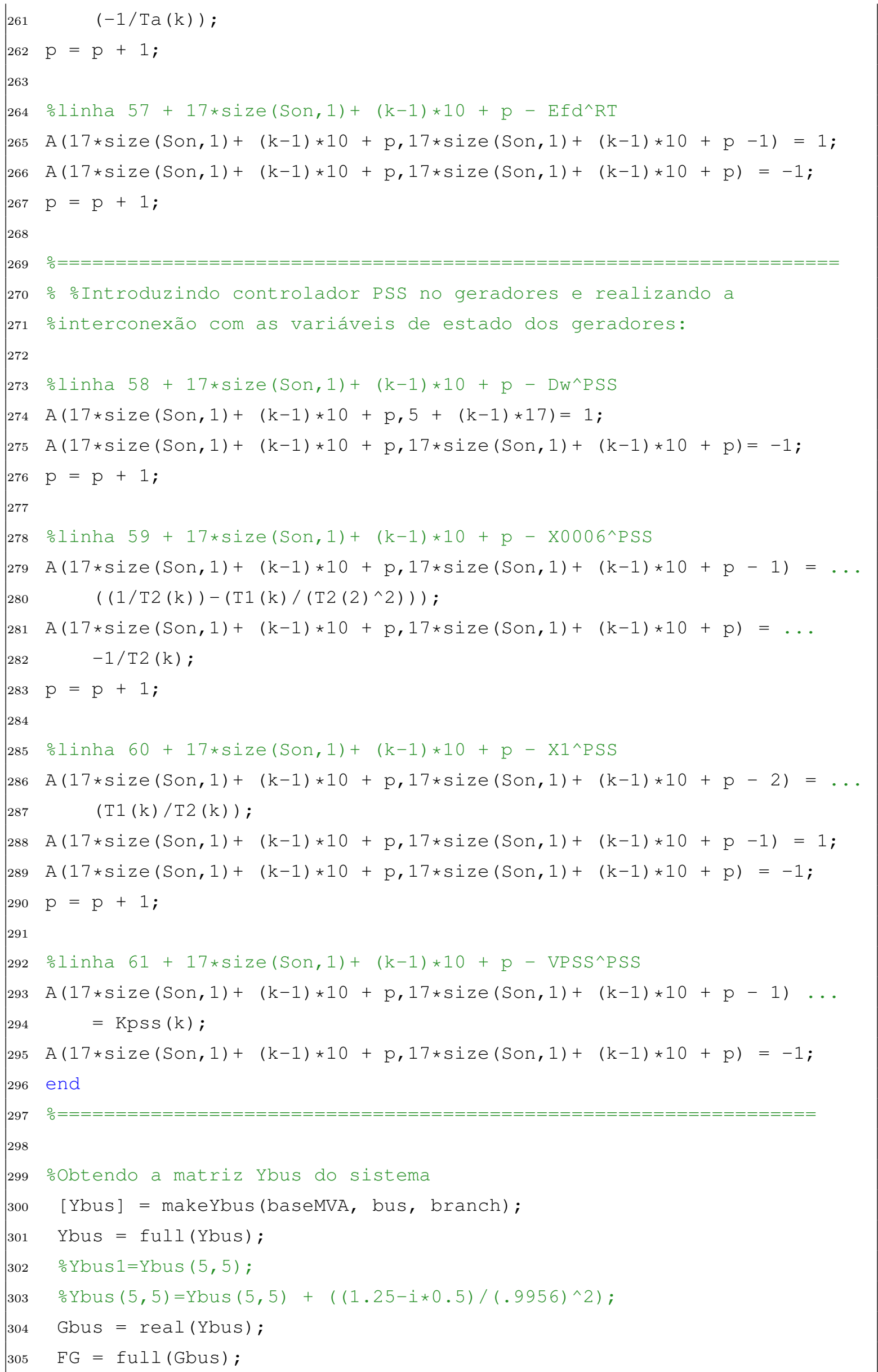




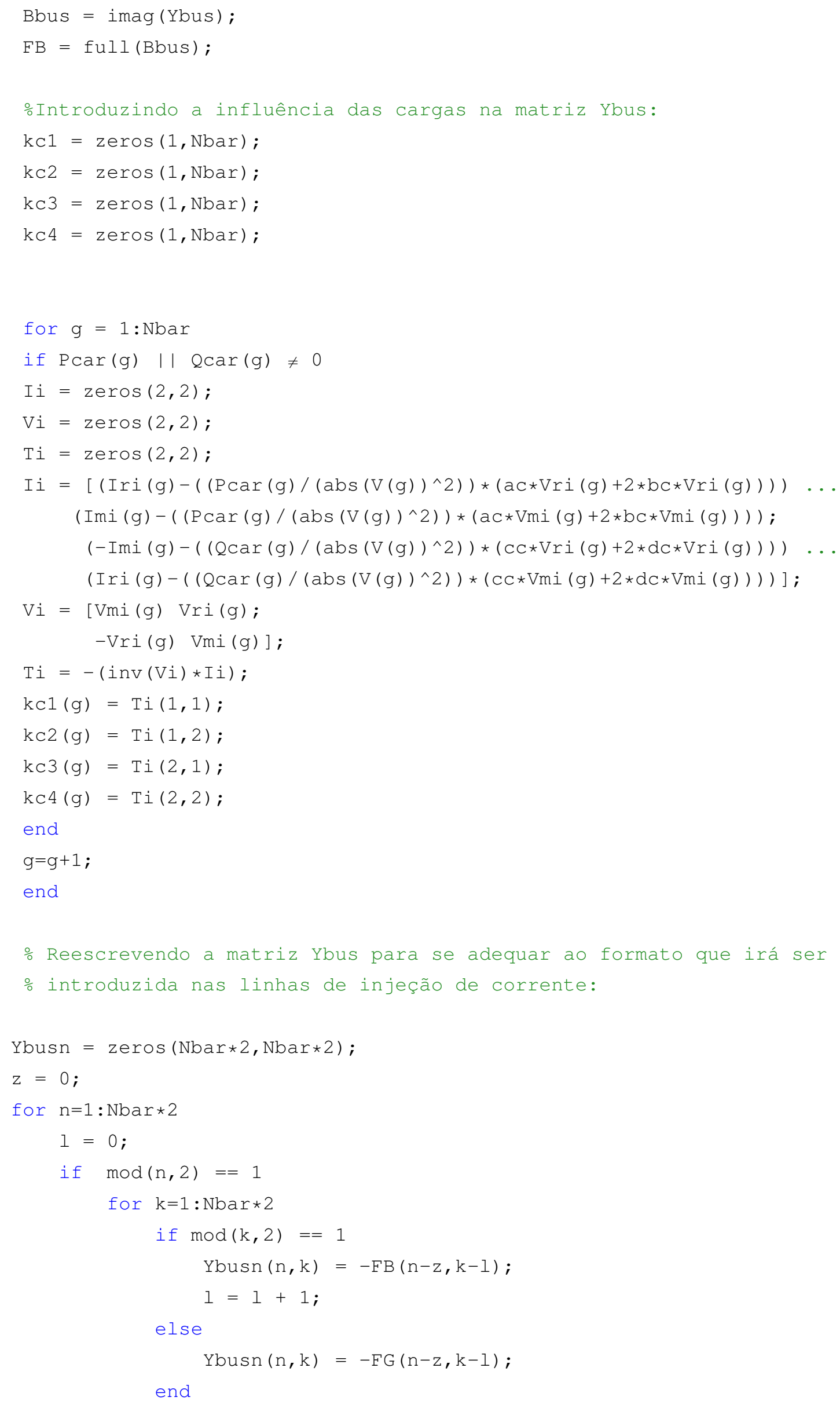




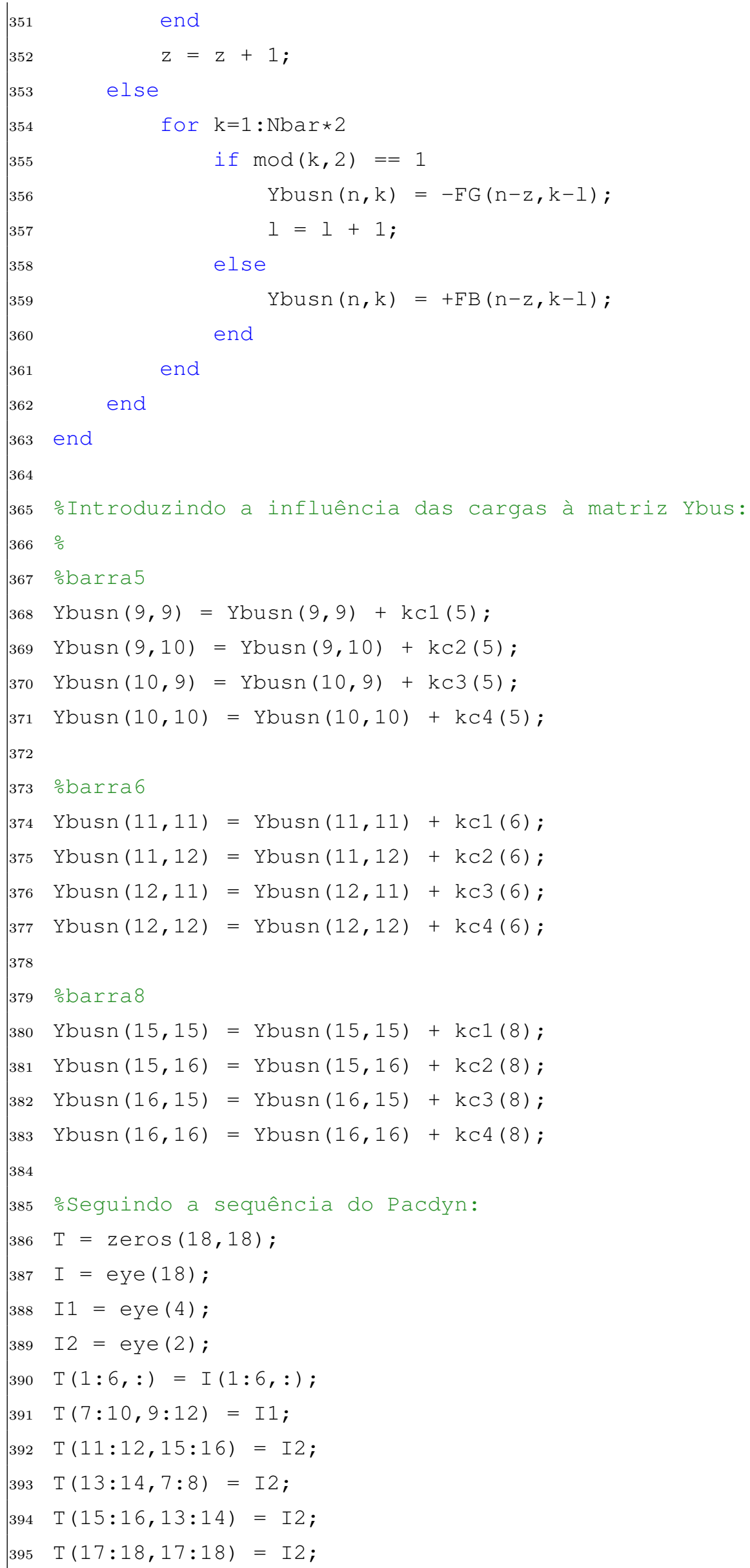




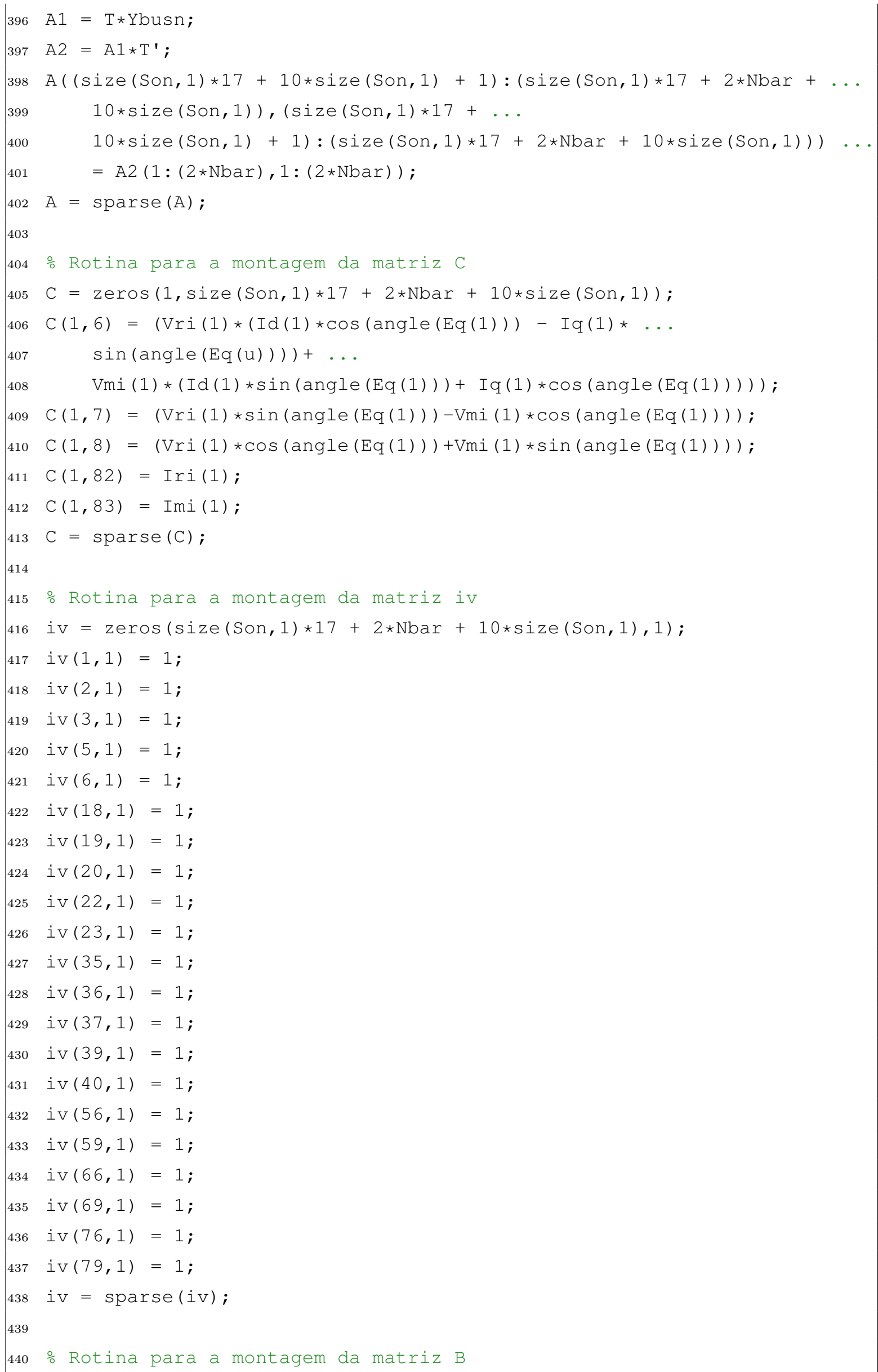




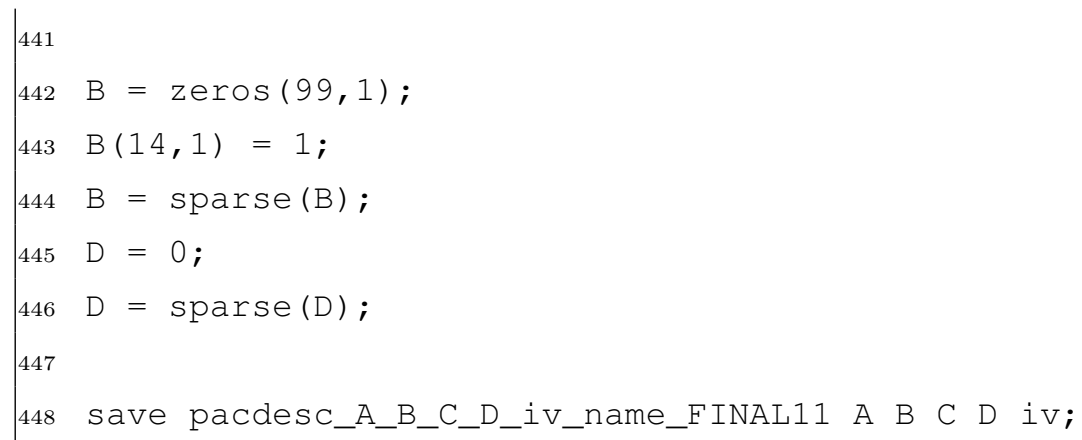

